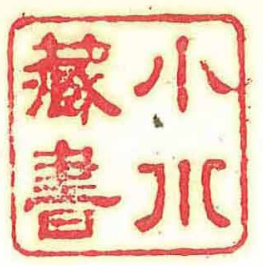

\title{
12. On the theory of linear translatable functional equations and Cauchy's series.
}

(Received June 17., 1936)

Tosio Kitagawa

Mathematical Institute, Osaka Imperial University.

CONTENTS.*

Introduction. .......

Chapter I. Expansion with respect to Linear Translatable Dif-

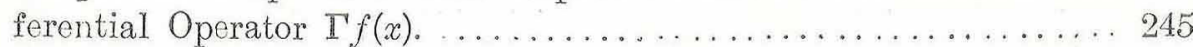

$\S 1$. Transformation of contour-integral. ............ 245

\& 2. Preliminary considerations................... 252

\& 3. Convergence-theorems of Cauchy's series. ........ 255

\$ 4. Asymptotic behaviour of an integral function and conditions of convergency in $\S 3 . \ldots \ldots \ldots \ldots \ldots 258$

\$ 5. Generating functions and their asymptotic representations. (Examples of the convergence-theorems). .. 268

Chapter II. Solutions of the Linear Translatable Functional Equation under Various Conditions.................... 271

\&6. Convergence-theorems and solutions of the functional equation. ......................... 271

$\$ 7$. Infinitely many time differentiable solutions and analytic solutions. ...................... 274

$\S 8$. The order of magnitude of solutions and Schürer's theorems. . .......................... 277

\& 9. The Ritt-Valiron's theory of linear differential equation of infinite order......................... 279

Chapter III. Fundamental Theorems of Cauchy's Series. ....... 282

\$10. Linear translatable operators to be considered. ...... 282

$\$ 11$. Integration, uniqueness, and differentiation of Cauchy's series................................. 283

\$12. A certain generalisation of Parseval's theorem...... 285

\$13. Multiplication-theorems. ................... 290

\$14. Integral-representation of the biorthogonal functions... 291 Chapter IV. General Linear Translatable Functional Equations

(") Some of the results, specially those of Chapter I, III and V, have been already communicated in the previous notes of the author. See Bibliography, 36. Kitagawa, T. [3]-[4]. 


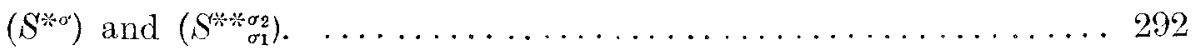

$\$ 15$. Transformation of contour-integrals with respect to the linear translatable differential operators $\Gamma^{*} f(x)$

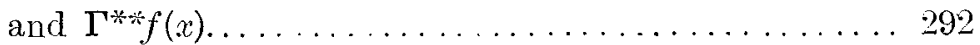

$\$ 16$. Preliminary considerations. . . . . . . . . . . 296

\$17. General linear translatable functional equations $(S * \sigma)$

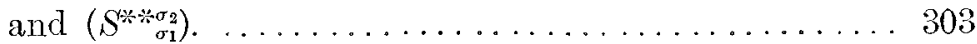

\$18. Applications of convergence-theorems. . . . . . . . . 305

\$19. Examples (singular integral equations). . . . . . . 306

Chapter V. Principal Solution of Non-homogeneous Linear Translatable Functional Equation. ..................... 307

$\$ 20$. Solution under integral representation. . . . . . . 307

\$21. Preliminary considerations. ............... 30 s

$\$ 22$. Introduction of generalised Bernoulli's polynomials... 310

$\$ 23$. Linear translatablity and $\phi$-polynomials of L. M. MilneThomson's. ....................... 314

$\$ 24$. Boundary value problem of linear homogeneous functional equation and Introduction of $\left\{\dot{B}_{k, \lambda_{0}}^{k}(x)\right\} . \ldots \ldots 315$

\$25. Summation-formula. .................. 318

$\$ 26$. Principal solution of non-homogeneous linear translatable equation. ...................... 320

\$27. Particular solution of non-homogeneous linear translatable equation......................... 323

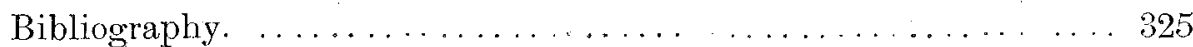

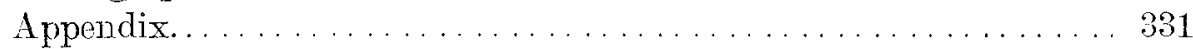

\section{Introduction.}

1. Numerous important researches concern themselves with linear functional operators which are permutable with differentiation or with translation $\left({ }^{1}\right)$, and various theories of functional equations allied to these operators have been established by many mathematicians. Thus, for examples, the theories of linear difference-differential equations with constant coefficients which we owe to Hilb, Schürer, Nörlund, Schmidt, Ghermanesco, Perron, Bochner, Carmichael, Wiener and others $\left({ }^{2}\right)$ are concerned with the linear operator

$$
\mathrm{E} f(x)=\sum_{\mu=0}^{n} \sum_{\nu=0}^{m} \alpha_{\mu, \nu} f^{(\mu)}\left(x+h_{\nu}\right), \quad(n \geqq 0)
$$

and those of some singular integral equations due to Bochner, and Hopf..

(1) Under a translation we understand a transformation which transforms $f(x)$ into $f(x+t)$, where $t$ is a certain real number.

( $\left.{ }^{2}\right)$ Cf. Hilb. [1], Schürer [1], Nörlund [1]-[2], Schmidt [1], Ghermanesco [1]-[5], Perron. [. 1]-[2] Bochner [2]-[5], Carmichael [1]-[3], Wiener [1]. 
Wiener $\left({ }^{3}\right)$ with

$$
\mathrm{B} f(x)=f(x)-\int_{-\infty}^{\infty} f(x+t) K(t) d t, \quad(-\infty<x<\infty)
$$

We may also mention here the Ritt-Valiron( ${ }^{*}$ ) theory of differential equation of infinite order with constant coefficients, for the transmutation given by

$$
\Lambda f(z)=\sum_{n=0}^{\infty} a_{n n} f^{(n)}(z), \quad\left(\left|z-z_{0}\right|<\rho\right),
$$

is also permutable with differentiation.

Besides these theories, it is well known that the Volterra theory $\left({ }^{5}\right)$ of permutable kernels and the Pincherle theory $\left({ }^{6}\right)$ of transformations of power series are appliciable to solve these functional equations.

These methods are extremely powerful and simple to use. Nevertheless, it will be noticed that they are subject to certain restrictions upon the range of their applications respectively. Thus the function to which some of the Laplace transformation, the Fourier transformation, and the generalised harmonic analysis of Hahn, Bochner, and Wiener $\left({ }^{7}\right)$ are applicable, are subject to severe conditions as to their behaviours at infinity. Such restrictions are not avoidable in the direct and simple methods developped along the lines of so-called operational calculus due to Heaviside, Carson, Doetsch, and others $\left({ }^{8}\right)$. On the other hand, the theories due to Volterra, Pincherle, Ritt, Perron, Valiron and others may be directly applicable to only analytic functions. The Fantappiè $\left({ }^{9}\right)$ theory of analytic functionals is one of the systematic and promising tools to handle with these functional equations, which, however, is also restricted to analytic functions.

We must, therefore, establish a new method which possesses a most large scope of the applications and which connects the above mentioned theories so intimately that we may convince ourselves with the skill and the power of them in their respective realms of applications.

For this purpose, we will attempt to develope the classical method of Cauchy's $\left({ }^{10}\right)$ which appeals to the residue theorem in theory of analytic functions and which was adopted by Hilb( ${ }^{11}$ ) in his theory of linear difference-

(3) Cf. Bochner [1], Hop-Wiener [1], Wiener [2]-[4], Hopf [1]-[2].

(4) Cf Ritt [1], Valiron [1], Bourlet [1]-[3], Davis [2]-[3], Scheffer [1]-[4], Pólya [1].

(5) Cf. Volterra [1]-[2].

( $\left.{ }^{6}\right)$ Cf. Pincherle [1]-[2].

( 7 ) Cf. Bochner [5], Wiener [3], Izumi [1], Tzumi and Kitagawa [1]-[3].

$\left({ }^{8}\right)$ Cf. Heaviside [1], Carson [1], Doetsch [3], Jeffreys [1], Mächler [1], March [1], Smith [1], Stacho [1].

( $\left.{ }^{9}\right)$ Cf. Fantappiè [1], Martis [1].

(10) Cf. Cauchy [1]-[2].

(11) Hilb [1]. 
differential equations, by Geppert, Nagumo and the author ( ${ }^{12}$ ) in their expansion-theories, by Dixon $\left({ }^{13}\right)$ in the theory of thin elastic plate, and recently by Delsarte ${ }^{*}$ ) in his theory of mean-periodic function.

To state our method and results in some details, we must begin with accurate definitions of the linear functional operators in our constant consideration and several fundamental notions which will be adopted in this paper.

2. Let us consider an operator $\Lambda$ which transforms a function $f(t)$ defined and mesurable in $(\alpha, \beta)$ into a function $\Lambda f(x)$ defined and mesurable in $\left(\alpha^{\prime}, \beta^{\prime}\right)$, where we assume that the interval $\left(\alpha^{\prime}, \beta^{\prime}\right)$ is contained in the interior of the interval $(\alpha, \beta)$, and which possesses the following propertics:

Property 1. For any fixed number $x$ in $\left(\alpha^{\prime}, \beta^{\prime}\right), \Lambda f(x)$ is a linear functional whose domain $E_{x}$ consists of functions defined and integrable on the set $\mathfrak{M}_{x}$, where $\mathfrak{M}_{x}$ is a subset of the interval $(\alpha, \beta)$ : that is, if we write more accurately

(0.04) $\left.\quad \Lambda f(x)=\Lambda\left|\left[x ; f(t): t \varepsilon \mathfrak{M}_{n}\right]\right|_{, .}{ }^{15}\right)$

then, for any two functions $f_{1}(x)$ and $f_{s}(x)$ in its. domain and any two (real or complex) numbers $c_{1}$ and $c_{2}$, we have

$$
\begin{aligned}
\Lambda\left|\left[x ; c_{1} f_{1}(t)+c_{2} f_{2}(t): t \varepsilon \mathfrak{M}_{x}\right]\right|= & c_{1} \Lambda\left|\left[x ; f_{1}(t): t \varepsilon \mathfrak{M}_{x}\right]\right| \\
& +c_{2} \Lambda\left|\left[x ; f_{:}(t): t \varepsilon \mathfrak{M}_{x}\right]\right| .
\end{aligned}
$$

Property II. $\mathbf{A} f(x)$ is permutable with translation: that is, whenever $x$ and $x+\tau$ belong to the interval $\left(\alpha^{\prime} ; \beta^{\prime}\right)$, then $\mathfrak{M}_{x}$ and $\mathfrak{M}_{x++}$ belong to the interval $(\alpha, \beta)$, and we have

(0.06) $\quad \mathbf{\Lambda}\left|\left[x+\tau ; f(t): t \varepsilon \mathfrak{M}_{x+\tau}\right]\right|=\mathbf{\Lambda}\left|\left[x ; f(\tau+t): t \varepsilon \mathfrak{M}_{x}\right]\right|$.

We call such operator $\Lambda$ as a linear translatable operator $\left({ }^{16}\right)$.

Here let it be noticed that, if we put $x=\xi_{0}$ and substitute $\tau$ by $x-\xi_{0}$ in $(0.06)$, then we get

(0.07) $\quad \mathbf{\Lambda}\left|\left[x ; f(t): t \varepsilon \mathfrak{M}_{i}\right]\right|=\Lambda\left|\left\lceil\xi_{0} ; f\left(x-\xi_{0}+t\right): t \varepsilon \mathfrak{M}_{\xi_{0}}\right]\right|$

Without loss of generality, we may assume that the initial point $\xi_{0}$ coincides with the origin, so that $(0: 07)$ becomes

(0.08) $\left.\quad \Lambda \mid\left[x ; f(t): t \varepsilon \mathfrak{M}_{x}\right]\right\}=\mathbf{\Lambda}\left|\left[0 ; f(x+t): t \varepsilon \mathfrak{M}_{0}\right]\right|$.

Therefore we adopt the abbreviate notation of the expression (0.04):

$$
\Lambda f(x) \equiv \Lambda_{0}\left\{f^{\prime}(x+t)\right\}
$$

(12) Cf. Geppert [1], Nagumo [1] Kitagawa [2].

(13) Cf. Fiiton [1], Dixon [1]-[2].

(14) Delsarte [1]-[5].

(15) For this notation, see Volterra [1] Introduction.

(16) This operator has been cal'ed by Volterra [1] as an operator of closed cycle, and by Benz [1] as "lineaire, verschiebungstreue Funkt:onaloperation." In Chapter I we assume that $\mathrm{E}_{x}$ contains all the functions which are continuous over $\mathfrak{M}_{x}$. 
In the study of the linear translatable operators, the functions $e^{\lambda t}(\lambda$ being complex in general) play an important rôle. This results from the fact that

$$
e^{\lambda(x+t)}=\grave{e}^{\lambda x t} \cdot e^{\lambda}
$$

or in the other words, that $e^{\lambda t}$ is a linear representation of translation. group of real numbers. Thus, if $e^{\lambda t}$ belongs to the domain of $\Lambda$, then the application of $\Lambda$ to $e^{\lambda t}$ yields us, in view of $(0.08)$ and $(0.10)$,

$$
\Lambda_{t}\{f(x+t)\}=\Lambda \mid\left[0 ; e^{\lambda x} \cdot e^{\lambda t}: t \varepsilon \mathfrak{M}_{0} \|\right.
$$

By Property I, we may put $e^{\lambda x}$ out of $\Lambda$, and we have

$$
\Lambda e^{\lambda x}=\boldsymbol{\Lambda}_{t}\left\{e^{\lambda(x+t)}\right\}=e^{\lambda x} \Lambda\left|\left[0 ; e^{\lambda t}: t \varepsilon \mathfrak{M}_{0}\right]\right|=e^{\lambda x} G(\lambda) \text {, say. }
$$

We call the function of $\lambda, G(\lambda)$, the generating function of the operator $\Lambda$. One of the essential points in our study is to classify the linear translatable operators as to the behaviours of their generating functions.

We are now in position to specify the operators and to explain what we discuss in each chapter.

3. In the first three chapters of this paper we assume the domain $E_{x}$ introduced in Property $\mathrm{I}$ is a complex space of the type $(F)$ in the Banach sense with or without the norm $\mid i f(t) \|_{x}$ for each fixed $x$ in $\left(\alpha^{\prime}, \beta^{\prime}\right)$, the set $\mathfrak{M}_{0}$ being taken as the closed finite interval $(a, b)$, where $a<0<b$, and we assume the following two additional properties:

Property III.( ${ }^{17}$ ) The operation $\Lambda$ is permutable with the definite Lebesque integration; that is to say, if a $L$-. $\left.{ }^{15}\right)$ integrable function $f(t)$ belongs to the domain of $\Lambda$, and if $x, t, \alpha^{\prime \prime}$ and $\beta^{\prime \prime}$ are subject to the conditions that the interval $\left[t+\alpha^{\prime \prime}, t+\beta^{\prime \prime}\right]$ belongs to $\mathfrak{M}_{x}$ and that $\alpha<x+\alpha^{\prime \prime}<x+\beta^{\prime \prime}<\beta$, then we have

$$
\Lambda\left|\left[x ; \int_{\alpha^{\prime \prime}}^{\beta^{\prime \prime}} f(t+\xi) d \xi: t \varepsilon \mathfrak{M}_{x}\right]\right|=\int_{\alpha^{\prime \prime}}^{\xi \prime \prime} \Lambda\left[x+\xi ; f(t): t \varepsilon \mathfrak{M}_{x+\xi}\right] \mid d \xi
$$

Further, if a sequence of continuous functions $\left\{f_{n}(t)\right\}\left(f_{n}(t) \subseteq E_{x}\right)$ tends to zero uniformly in $\mathfrak{M}_{x}$ as $n \rightarrow \infty$, then $\Lambda f_{n}(x)$ tends to zero as $n \rightarrow \infty$.

Property IV. The generating function $G(\lambda)$ of the operator $\Lambda f(x)$ is an integral function of $\lambda$.

It may be not unnecessary to show examples of the linear translatable operators which satisfy the above four properties:

$1^{\circ}$ Let us consider a space consisted of bounded and continuous func-

(17) This may be recognised as a continuity property of $\Lambda$, for the permutability of $\mathrm{V}$ with the integration may be verified by properties $I$ and $I I$ by imposing suitable conditions upon the continuity of $\Lambda$. For the sake of distinctness and emphasis, we sometimes explicitly state this Property III on the Hypothesis of our Theorems.

(18) In this paper, for the sake of brevity, we merely say " $I$-integrable" or "integrable" instead of "Lebesgue-integrable." 
tion defined in $\left(\alpha^{\prime}+a, \beta^{\prime}+b\right)$, and let

$$
\|f(t)\|_{x=\underset{x+a \leqq t \leqq x+b}{\operatorname{Max}} \mid}|f(t)|
$$

and

$$
\Lambda f(x)=\int_{a}^{b} f(x+t) d \varphi(t),\left({ }^{19}\right)
$$

where $\varphi(t)$ is a function of bounded variation in $(a, b)$. Then, wo hare

$$
|\Lambda f(x)| \leqq\|f(t)\|_{x} \int_{a}^{b}|d \varphi(t)|,
$$

Properties I-IV are satisfied.

$2^{\circ}$ Let us consider a space $L^{\prime \prime}\left(a+\alpha^{\prime}, b+\beta^{\prime}\right)$, and let $K(x)$ belong to $L^{q}(a, b),\left(\frac{1}{p}+\frac{1}{q}=1\right)$.

Let us put

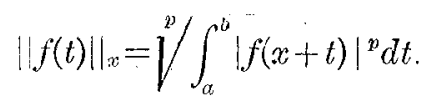

Then the liner translatable operator

$$
\Lambda f(x)=\int_{a}^{b} f(x+t) K(t) d t
$$

possesses the Properties $I-V$. Hore we have

$$
|\Lambda f(x)| \leqq\left.\left.\|f(x)\|\right|_{x}\right|^{q} \int_{a}^{\bar{b}}|K(t)|^{q} d t
$$

$3^{\circ}$ Let the generating function of the transmutation $(0.03)\left({ }^{20}\right)$

$$
G(\lambda)=\sum_{n=0}^{\infty} a_{n} \lambda^{n}
$$

be at height of the mean type of the order 1, and let the least upper bound of $\gamma$ such that

$$
|G(\lambda)|<e^{\gamma|\lambda|}
$$

be denoted by $\Gamma$. Let $E_{z}$ be the set of all regular and analytic functions defined at least in the circle $\mathfrak{S}_{z}$ defined by $|\xi-z| \leqq \Gamma+h$, where $h$ is a positive constant independent of $z$. Then $E_{z}$ becomes a normalised spaco if we define the norm of $f(\xi) \varepsilon E_{z}$ by

$$
\|f(\xi)\|_{z}=\operatorname{Max}_{\xi \in \complement_{z}}|f(\xi)| \text {. }
$$

Let us consider the set of $z$ belonging to $\left|z-z_{0}\right| \leqq h^{\prime}$.

Then, by Valiron's theorem (Valiron [1] p. 35), there is a constant $M$ dependent of $h^{\prime}$ and of $G(\lambda)$ and independent of $f(z)$ such that in $\left|z-z_{0}\right| \leqq h^{\prime}$

(19) This contains as its special case $\Delta f(x) \equiv f(x+b)-f(x+a)$.

$\left({ }^{20}\right)$ See $\$ 9$ in Chapter II and Appendix I. 


$$
|\Lambda f(z)| \leqq M\|f(\xi)\|_{z}
$$

Therefore the transmutation $(0.03)$ is one of the linear translatable operator now in consideration. It is defined for the complex analytic function of the complex domain, and satisfies the properties defined for real arguments: inded, we have only to consider the segment parallel to the real axis, passing through the point $z$.

The object of our attack in this chapter is a linear translatable differential operator $\Gamma f(x)$, which transforms a function $f(x)$ defined and $n$-time differentiable in $\left(x_{0}+a, x_{0}+b\right)$ into a function $\Gamma f(x)$ defined and integrable in $(\alpha, \beta)$, and which is defined by

$$
\Gamma f(x)=\sum_{k=0}^{n} \Lambda^{k} f^{(k)}(x)
$$

where $\Lambda^{k}(k=0,1,2, \ldots, n)$ are the liner translatable operators in our consideration, $n$ being non-negative integer.

The generating function $G(\lambda)$ of the operator $\Gamma$ becomes

$$
G(\lambda)=\sum_{k=0}^{n} \lambda^{k} G_{k}(\lambda)
$$

where $G_{k}(\lambda)$ are the gencrating function of $\Lambda^{k}$.

By $\left\{\lambda_{n}\right\}$ we denote the zero-points of the integral function $G(\lambda)$ arranged in the order of magnitude of the absolute values and amplitudes. Wo consider the series (formally defined)

$$
Q_{0}(x)+\sum_{m=1}^{\infty} Q_{m}(x) e^{\lambda_{m}} x
$$

where $Q_{m}(x)(m=0,1,2, \ldots)$ are the polynomials whose degrees are less than the multiplicities $\left({ }^{21}\right)$ of the corresponding $\lambda_{m}\left({ }^{22}\right)$.

We adopt the following

Definition I. The series (0.26) is called the exponential series with respect to the linear translatable operator $\Gamma$.

In Chapter I we will treat the expansion theorem of arbitrary functions $f(x)$ defined and at least $n$-time differentiable in $\left(x_{0}+a, x_{0}+b\right)$ into the exponential series with respect to $\Gamma$, where $x_{0}, a(<0)$, and $b(>0)$ are assigned real numbers subject to the condition that $\mathfrak{M}_{0} \cong(a, b)$.

For this purpose we consider a contour-integral.

$$
(0.27) \quad \mathrm{T}_{r}\left(x, x_{0} ; f\right) \equiv \frac{1}{2 \pi i} \oint_{\mathbb{E}_{r}} \frac{e^{\lambda x}}{G(\lambda)} \sum_{k=0}^{n} \Lambda_{0}^{k}\left\{e^{\lambda \xi} \int_{x_{0}}^{x_{0}+\xi} e^{-\lambda \eta} f(\eta) d \eta\right\} d \lambda
$$

\footnotetext{
(21) The multiplicity of $\left\{\lambda_{n}\right\}$ is the positive integer $k$ such that

$$
G^{(s)}\left(\lambda_{n}\right)=0 \quad(s=0,1,2, \ldots, k-1)
$$$$
G^{(k)}\left(\lambda_{n}\right) \neq 0
$$

(22) We put $\lambda_{0}=0$. If $\lambda_{0}=0$ is not the zero-point of $G(\lambda)$, then $Q_{0}(x)$ does not exist. 


$$
+\frac{1}{2 \pi i} \oint_{\mathbb{C}_{,}} \frac{e^{\lambda\left(x-x_{0}\right)}}{G(\lambda)} \sum_{k=1}^{n} \lambda^{k} G_{k}(\lambda) \sum_{\nu=0}^{k-1} \frac{f^{(\nu)}\left(x_{0}\right)}{\lambda^{\nu+1}} d \lambda
$$

where we suppose that there is no zero of $G(\lambda)$ on the curve $\mathfrak{E}_{r}$. As easily verified, this contour-integral possesses the following properties:

$1^{\circ}$. If $f(x)=c_{1} f_{1}(x)+c_{2} f_{2}(x)$ in $x_{0}+a<x<x_{0}+b$, then we have

(0.28) $\quad \mathrm{T}_{r}\left(x, x_{0} ; f\right)=c_{1} \mathrm{~T}_{r}\left(x, x_{0} ; f_{1}\right)+c_{2} \mathrm{~T}_{r}\left(x, x_{0} ; f_{2}\right)$.

$2^{\circ}$. If specially $f(x)=t^{s} e^{\mu t}$, $s$ being any non-negative integer and $\mu$ being any real or complex number, then direct computation yields un

$$
\mathrm{T}_{r}\left(x, x_{0} ; f\right)=\frac{\partial^{s}}{\partial \mu^{s}}\left[\frac{e^{\mu x_{0}} G(\mu)}{2 \pi i} \oint_{\mathbb{C}_{r}} \frac{e^{\lambda\left(x-x_{0}\right)}}{(\mu-\lambda) G(\lambda)} d \lambda\right]+x^{s} e^{\mu x} .
$$

$3^{\circ}$. By $1^{\circ}$ and $2^{\circ}$, it follows that if $f(x)$ is an exponential series with respect to $\Gamma$ with finite summanden, that is to say,

$$
f(x)=Q_{n}(x)+\sum_{u=1}^{N} e^{\lambda} n^{x} Q_{n}(x)
$$

then we have

$$
\mathrm{T}_{r}\left(x, x_{0} ; f\right)=f(x) .
$$

On this account we will adopt

Definition II $\left({ }^{23}\right)$. The exponential series with respect to $\Gamma$ defined by the contour-integral $\mathrm{T}_{r}\left(x, x_{0} ; f\right)$ is called a section of Cauchy's series of $f(t)$ with respect to the operator $\Gamma$ at the point $x_{0}$.

Thus arises the problem whether any arbitrary function $f(x)$ may be represented as the limit of $\mathrm{T}_{r}(x, x, f)$ (as $r \rightarrow \infty$ ) or not, where the sequence of contours $\left\{\mathfrak{E}_{r}\right\}$ is assumed to bo selected such that $\mathfrak{E}_{r}$ is contained in the domain enclosed by $\mathfrak{E}_{r+1}$ and $\mathfrak{E}_{r}$. diverges to the whole plane as $r \rightarrow \infty$, that is, the distance between the origin and $\mathfrak{E}_{r}$, which we denote by $d_{r}$, tends to infinity as $r \rightarrow \infty$.

We will answer to this problem by estimating the contour-integral. Our answer to this problem is affirmative in the sense that under suitable choice of the sequence of contours $\left\{\mathbb{E}_{r}\right\}$ the expansion of arbitrary functions defined in $\left(x_{0}+a, x_{0}+b\right)$ into the Cauchy's series with respect to the operator $\Gamma$ is possible if the generating function $G, \lambda$ ) satisfies certain conditions and if $f(x)$ belongs to certain class of functions.

In the estimation of $(0,27)$, we suppose that $f(x)$ belongs to one of the following classes.

$1^{\circ}$. Let $m$ be a non-negative integer. The class $\mathscr{L}_{m}\left(x_{0}+a, x_{0}+b\right)$ $\left(=\mathscr{L}_{m}\right)$ consists of functions $f(x)$ defined and $m$-time differentiable in the

( $\left.{ }^{23}\right)$ For this terminology, see Fejes [1]. The series corresponds to what Ritt-Valiron called Dirichlet's series in their theories of linear differential equation of infinite order. 
interval $\left(x_{0}+a, x_{0}+b\right)$ such that

(0.31) $f^{(m)}(x)$ is Lebesgue-integrable over $\left(x_{0}+a, x_{0}+b\right)$.

$2^{\circ}$. The class $V_{m}\left(x_{0}+a, x_{0}+b\right)\left(=V_{m}\right)$ is a sub-class of $\mathscr{L}_{m}$ such that

$(0.32) f^{(m)}(x)$ is of bounded variation on $\left(x_{0}+a, x_{0}+b\right)$.

It will be shown that the choice of $\left\{\mathbb{E}_{r}\right\}$ in $(0.18)$ depends upon the estimation of the following integrals:

$$
\int_{\mathbb{C}_{r}+e^{(+)}}\left|e^{-\lambda \delta_{2}}\right| d \lambda \mid
$$

for some $\delta_{3}(<0)$,

$$
\int_{(\mathbb{E}, r}\left|e^{\lambda \delta 1}\right||d \lambda|
$$

for some $\delta_{1}(>0)$, and

$$
\int_{\mathfrak{C}_{r}, \pm} \frac{e^{\lambda_{I}}}{\lambda^{m} G(\lambda)}|d \lambda|
$$

$q$ being a real number, $m$ being an integer, $\mathbb{S}_{r}^{(+)}$and $\mathfrak{S}_{r}^{(-)}$being the parts of $\mathfrak{E}$. belonging to the right and the left half-planes, respectively.

We will give this reduction in $\$ 2$, from which we obtain a group of convergence theorems concerning the expansion in Cauchy's series with respect to the operator $\Gamma$, which will be shown in $\$ 3$. These considerations and results make it adequate and necessary to introduce the notion of the "Association" of the generating function with respect to the sequence of th: contours $\mathbb{E}_{r}\left({ }^{*}\right)$ and to certain interval. This idea plays the important rôle in our whole study.

In $\$ 4$ we consider the generating functions posseswing certain asymptotic representations, and give the construction of the sequences of contours satisfying the conditions of convergences given in $\$ 3$.

In $\& 5$ we will give concrete examples of our convergence-theorems. We show that our results obtained in this chapter contain the classical expansion-theorems of Fourier's series and of Hilb's research on differencedifferential equations as their special cases. We will also remark here that the convergence theorem in the Delsarte theory of the mean-periodic function( $\left.{ }^{25}\right)$ belongs to ours as one of the important special cases.

4. In Chapter II we devote our attention to the functional equation

$$
\Gamma f(x) \equiv \Gamma_{0}\left\{f^{\prime}(x+t)\right\}=0, \quad\left(x_{0}+\alpha<x<x_{0}+\beta\right) .
$$

The direct application of the convergence-theorems obtained in the

(24) Accurate definition will be given in $\S 3$

(96) Delsarte [5] p. 444-445. 
previous chapter yields us the expansion-theorems of tho solutions, as will be shown in $\$ 6$. Further in $\$ 7$ wo will modify these results so that we may obtain the corresponding theorems in the theory of the linear differential equations of infinite order, whose gencrating function is of the order zero.

In $\$ 8$ we take in consideration those solutions which are subject to certain conditions as to their behaviours at infinity. The interesting theorem due to Schürer [1] will be proved by another way.

Since our method is quite different from those which we owe to Ritt [1], Valiron [1], Sheffer [2], Davis [3] and others, it may be worth while to give a simple illustration to their methods, which we will sketch in $\$ 9$.

5. In Chapter III our chief purpose is to give fundamental theorems of Cauchy's series defined for the functions belonging to $\left(\mathscr{L}_{0}\right)$ with respect to the linear operator $\Lambda f(x)$ defined in Chapter I, which may be recognised as extension of those of classical Fouricr's series which is certainly a special case of Cauchy's series.

We consider, throughout this chapter, certain special linear translatable operators whose accurate definition will be stated in $\$ 10$. In $\$ 11$ we discuss the fundamental operations i. e. differentiation and integration imposed upon of Cauchy's series, and also obtain uniqueness-theorem of that series.

In 12 we will make a certain generalisation of Parseval's theorcm well-known in Fourier's scries to Cauchy-series. For this purpose we will restrict ourselves to the linear translatable operator with certain condition which will be assumed only in $\$ 12$.

In $\$ 13$ we will mention a multiplication-theorem of two Cauchy'sseries with respect to the same linear translatable operators. In $\$ 14$ an integral-representation of biorthogonal function system determined by the lincar translatable operation will be given.

6. In Chapter IV we turn to the consideration of the linear translatabl. operators which are more general than those considered in the previous three chapters in the sense that we do not assume here that their generating functions are integral functions. There are two fundamental operations now in consideration.

One of them will be denoted by $\Lambda^{*} f(x)$. It possesses the following three properties:

Property I.* For every fixed number $x$ in the interval $(0, \infty), \Lambda^{*} f(x)$ is a linear functional whose domain consists of functions which are defined in $(0, \infty)$, integrable in any finite range, and are of the finite right "Stufe" 
$\alpha$, where $\alpha$ is less than a certain fixed number $\sigma \cdot\left({ }^{26}\right)$

Property II.* $\Lambda^{*} f(x)$ is permutable with any translation of positive argument: that is, if we write

$$
\mathrm{T}_{\tau} f(x) \equiv f(x+\tau)
$$

then we have, for any real positive number $\tau$,

$$
\Lambda * \mathrm{~T}_{\tau} f(x)=\mathrm{T}_{\tau} \Lambda^{*} f(x) .
$$

Property III.* The generating function $G^{*}(\lambda)$ of the operator $\Lambda^{*} f(x)$ $i s$ defined at least in a certain half-plane $R(\lambda) \leqq \sigma$ and is regular in every half-plane $R(\lambda)<\sigma-\varepsilon$, where $\varepsilon$ is any positive number.

The other operator, which we denote by $\Lambda^{* *} f(x)$, possesses the following properties:

Property I.** For any fixed number $x$ in $(-\infty, \infty), \Lambda^{* *} f(x)$ is a linear functional whose domain consists of functions which are defined in $(-\infty, \infty)$, integrable in any finite range, and are of the finite "Gesamtstufe" $(\alpha, \beta)$, where $\alpha$ and $\beta$ are subject to $\sigma<\alpha \leqq \beta<\sigma_{:}, \sigma_{1}$ and $\sigma_{2}$ being certain fixed real numbers.

Property II.** $\Lambda^{* * *} f(x)$ is permutable with translation $\mathrm{T}_{\tau} f(x)$, where $\tau$ is any real number.

Property III.** The generating function $G(\lambda)$ is defined at least in the strip $\sigma_{1} \leqq R(\lambda) \leqq \sigma_{2}$ and is regular in every strip $\sigma_{1}+\varepsilon<R(\lambda)<\sigma_{2}-\varepsilon$, where $\varepsilon$ is any positive number.

The object of Chapter IV is to solve the following two functional equations

and

$$
\Gamma^{*} f(x) \equiv \sum_{k=0}^{n} \Lambda^{* k} f^{(k)}(x)=0, \quad(0 \leq x<\infty)
$$

$$
\Gamma^{* *} f(x) \equiv \sum_{k=0}^{n} \Lambda^{* * k} f^{(k)}(x)=0, \quad(-\infty<x<\infty)
$$

where $\Lambda^{* k}$ and $\Lambda^{* * k}(k=0,1,2, \ldots n)$ are the linear translatable operators defined just now.

("6) Under the right "Stufe" we understand the number

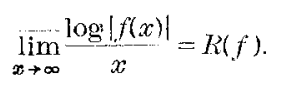

Similary we define the left "Stufe":

$$
\lim _{x \rightarrow-\infty} \frac{\log |f(x)|}{-x}=l(f)
$$

and also the "Gesamtistufe" we define as the par of the numbers

$$
(L(f), R(f)) \text {. }
$$

See, Bochner [3], §3. Ein Hilfssatz: 4 S. 432. 
For this purpose we appeal to the contour-integrals $\mathrm{T}_{4}^{*}\left(x, x_{0} ; f\right)$ and $\mathrm{T}_{\alpha}^{* *}\left(x, x_{0} ; f\right)$ which are the modified forms of the original integral $\mathrm{T}_{r}\left(x, x_{0}\right.$; f) suitably chosen so that we may easily secure the convergence of the sequences of them. The decompositions into several simpler integra's will be performed with respect to these contour-integrals in quite parallel mamer in their principle to those in $\$ 1$, although some different devices will be necessary on account of the fact that $\left\{\mathbb{E}_{r}\right\}$ should be restricted to the domain of the existence of the generating function.

Before we reach the final results of this chapter we are obliged to make further assumptions on $\Lambda^{*} f(x)$ and $\Lambda^{* *} f(x)$, which we term the properties $\left(N^{* 3}\right)$ and $\left(U^{* \beta}\right)$, and $\left(N_{a}^{* * \beta}\right)$ and $\left(U^{* * \beta}\right) \cdot\left({ }^{27}\right)$ Neverthless it will be easily verified that our results obtained in $\$ 17$ aro large enough to contain some theorems well known in the theories of difference equations and singular integral equations, as will be shown in $\$ 18$ and $\$ 19$.

7. In Chapter $\mathrm{V}$ we will generalise the Nörlund theory of the finite difference equation

$$
\Delta f(x) \equiv f(x+1)-f(x)=g(x), \quad(0 \leqq x<\infty)
$$

to the non-homogeneous linear translatable functional equation

$$
\Gamma f(x)=g(x), \quad(0 \leqq x<\infty),
$$

which contains the former as its special case.

Although his theory has been recently generalised to the ipecial equation of $(0.45)$, i.e,

$$
\mathrm{E} f(x) \equiv \sum_{i \sim 0}^{n} A_{i} f\left(x+\alpha_{i}\right)=g(x), \quad(0 \leqq x<\infty),
$$

by many mathematicians $\left(^{-8}\right)$, it seems to us that none of them has not yet given the principal solution under the form analogous to that of Nörlund's. Therefore we will endevour to define this in $\$ 26$, and we will term the solutions which correspond to those obtained by these authors the particular solutions. Except this definition, the results are quite parallel to those which we owe to the four authors, but it may be worth while to remark that our constant emphasis is laid on the fact that the whole theory is nothing but one of the somewhat practical method of dealing with the contour-integrals $N_{r}\left(x, x_{0} ; g\right)$ which will be introduced in $\$ 12$. Further the convergence-theorems obtained in $\$ 3$ make great service to the boundary value problems of the functional cquation. Here the notion of the asssociation of the generating function to certain interval introduced in $\$ 3$

(27) These properties of $\Lambda^{*}$ and $\Lambda^{* *}$ correspond to the continuity property of $A$, that is to say, Property III. See $\$ 16$ in Chapter IV.

(23) Pincherle [3], Bochner [2], Ghermanesco, [1]-[6], Raclis [1]. Here we notice that the Nörlund's principal solution possesses the characteristic properties. See Nörlund [2] p. 59. 
plays the important part; any interval of that length may be recongnised as the fundamental interval where the value of the solutions of the functional equation may be arbitiarily given. Here we wish to propose the problem under what conditions the solutions which we call the principal solutions will become identical with each others and will possess the characteristic properties similar to those of Nörlund's principal solution on the the difference-equation (0.44).

The course and results of the arguments will lead us to the calculus of operators, in which the contour-integral will be metamorphed into the operator-equation, where the algebraic features of the linear translatable operators will become eminent. In the operator-calculus wo have no need of assuming the regularity of the generating function, and we may attack the functional equation even in the ease when $G(\lambda)$ is defined merely in the imaginary axis of $\lambda$-plane. Therefore it will be a matter of importance to make clear the relation between the contour-integral and the operatorcalculus, which the author will disceuss in another occasion.

In conclusion of this Introduction, the author wishes to express his gratitude for the kind advices of Prof. Tzumi and for the constant encouragements of Prof. Shimizu and of Prof. Nagumo during his work.

\section{Chapter I. Expansion with respect to Linear Translatable Differential Operator $\Gamma f^{\prime}(x)$.}

\section{$\$ 1$. Transformation of Contour-Integral.}

1. Let $f(x)$ be a function which belongs to $\mathscr{L}_{n}\left(x_{0}+a, x_{0}+b\right)$, where $x_{n}, a(<0)$ and $b(>0)$ are assigned real numbers, and let us consider the contour-integral

$$
\mathrm{S}_{r}\left(x, x_{0} ; f\right) \equiv \frac{1}{2 \pi i} \oint_{\mathcal{E},} \frac{e^{\lambda x}}{G(\lambda)} \sum_{i=0}^{n} \Lambda_{\xi}^{n}\left\{e^{\lambda \xi} \int_{x_{0}}^{x_{0}+\xi} e^{-\lambda \eta} f^{(k)}(\eta) d \eta\right\} d \lambda .
$$

We denote by $\mathfrak{C}_{r}^{(+)}$and $\mathfrak{E}_{r}^{(-)}$the parts of $\mathfrak{S}_{r}$ which belong to the positive and the negative half-planes in the $\lambda$-plane respectively, and we assume that there are merely two points of the intersections of $\mathfrak{S}_{r}$ with the imaginary axis of $\lambda$-plane, which we denote by $i \rho$, and $-i \rho_{r}$ respectively.

Now we decompose the integral $\mathrm{S}_{r}\left(x, x_{s} ; f\right)$ into two parts :

$$
\mathrm{S}_{r}\left(x, x_{0} ; f\right)=\frac{1}{2 \pi i}\left\{\int_{\substack{\mathfrak{C}_{r}(+) \\ r\left(\mathbb{C}_{r}(-)\right.}}+\int_{r}\right\} \mathrm{S}_{r}^{(+)}\left(x, x_{0} ; f\right)+\mathrm{S}_{r}^{(-)}\left(x, x_{0} ; f\right),
$$

say, where we suppose that $\mathfrak{E}_{r^{+}}^{(+)}$and $\mathfrak{E}_{r}^{(-)}$are oriented in the same way as $\mathfrak{S}_{r}$. By the linearity of $\Lambda$, we have

$$
\mathrm{S}_{r}^{(+)}\left(x, x_{0} ; f\right)=\frac{1}{2 \pi i} \int_{\mathfrak{C}_{r}^{(+)}} \frac{e^{\lambda x}}{G(\lambda)} \sum_{k=0}^{n} \Lambda_{\xi}^{k}\left\{e^{\lambda \xi_{0} \cdot} \int_{x_{0}}^{x_{0}+b} e^{-\lambda \eta} f^{(k)}(\eta) d \eta\right\} d \lambda
$$




$$
-\frac{1}{2 \pi i} \int_{\mathcal{E}_{\xi^{(+)}}^{(+)}} \frac{e^{\lambda x}}{G(\lambda)} \sum_{k=0}^{n} \Lambda_{\xi}^{k}\left\{e^{\lambda \xi} \int_{x_{0}+\xi}^{x_{0}+\delta} e^{-\lambda \eta} f^{(k)}(\eta) d \eta\right\} d \lambda
$$

Since we have, by integration by parts,

$$
\begin{aligned}
e^{\lambda \xi} \int_{: c_{0}}^{x_{0}+b} e^{-\lambda \eta} f^{(k)}(\eta) d \eta= & e^{\lambda \xi} \sum_{\nu=1}^{k} \lambda^{\nu-1}\left[e^{-\lambda \eta} f^{(k-\nu)}(\eta)\right]_{x_{0}}^{x_{0}+b} \\
& +\lambda^{k} e^{\lambda \xi} \int_{x_{0}}^{x_{0}+b} e^{-\lambda \eta} f(\eta) d \eta, \quad(k=1,2, \ldots, n),
\end{aligned}
$$

and then

(1.051)

$$
\begin{aligned}
\Lambda_{\xi}^{k}\left\{e^{\lambda \xi} \int_{z_{0}}^{x_{0}+b} f^{(k)}(\eta) d \eta\right\} & \\
= & \lambda^{k} G_{k}^{\gamma}(\lambda) \int_{x_{0}}^{x_{0}+b} e^{-\lambda \eta} f(\eta) d \eta \\
& +\lambda^{k} G_{k}(\lambda) \sum_{\nu=0}^{k-1} \frac{e^{-\lambda\left(x_{0}+b\right)} f^{(\nu)}\left(x_{0}+b\right)}{\lambda^{\nu+1}} \\
& \quad-\lambda^{k} G_{k}(\lambda) \sum_{\nu=0}^{k-1} \frac{e^{-\lambda x_{0}} f^{(\boldsymbol{\nu})}\left(x_{0}\right)}{\lambda^{\nu+1}}, \quad(k=1,2, \ldots, n),
\end{aligned}
$$

and

(1.052)

$$
\begin{aligned}
\Lambda_{\xi}^{k}\left\{e^{\lambda \xi} \int_{x_{0}+\xi}^{x_{0}+e^{-k}} e^{-\lambda \eta} f^{(k)}(\eta) d \eta\right\} & \\
= & e^{-\lambda x_{0}} \sum_{\nu=0}^{n-k-1} \frac{\Lambda_{\xi}^{k}\left\{f^{(k+\nu)}\left(x_{0}+\xi\right)\right\}}{\lambda^{\nu+1}} \\
& -e^{-\lambda\left(x_{0}+b\right)} G_{k}(\lambda) \sum_{\nu=0}^{n-k-1} f^{(k+\nu)} \frac{\left(x_{0}+b\right)}{\lambda^{\nu+1}} \\
& +\frac{1}{\lambda^{n-k}} \Lambda_{0}^{k}\left\{e^{\lambda \xi} \int_{x 0+\xi}^{x_{0}+b} e^{-\lambda \eta \eta} f^{n)}(\eta) d \eta\right\}, \quad(k=0,1,2, \ldots, n-1) .
\end{aligned}
$$

(1.03) becomes

(1.06) $\quad \mathrm{S}_{r}^{+}\left(x, x_{0} ; f\right)$

$$
\begin{aligned}
& =\frac{1}{2 \pi i} \int_{\mathscr{C}_{r}^{(+)}} e^{\lambda x}\left(\int_{x_{0}}^{x_{0}+b} e^{-\lambda \eta} f(\eta) d \eta\right) d \lambda \\
& -\frac{1}{2 \pi i} \int_{\mathbb{S}_{r}^{(+)}} \frac{e^{\lambda\left(x-x_{0}\right)}}{G(\lambda)} \sum_{k=1}^{n} \lambda^{\prime} G_{k}(\lambda) \sum_{\nu=0}^{k-1} \frac{f^{(\nu)}\left(x_{0}\right)}{\lambda^{\nu+1}} d \lambda \\
& +\frac{1}{2 \pi i} \int_{\left(S_{,}^{\prime},+\right)} \frac{e^{\lambda\left(x-x_{0}-b\right)} G(\lambda)}{G(n)} \sum_{k=0}^{n} \lambda^{i} G_{k}^{\prime}(\lambda) \sum_{\nu=0}^{n-1} \frac{f^{(p)}\left(x_{0}+b\right)}{\lambda^{p+1}} d \lambda \\
& +\frac{1}{2 \pi i} \int_{\left.\mathbb{C}_{r^{\prime}}+\right)} \frac{e^{\lambda\left(x-x_{0}\right)}}{G(\lambda)} \sum_{k=0}^{n-1} \sum_{\nu=0}^{n-k-1} \frac{\Lambda_{\xi}^{k}\left\{f^{(k+\nu)}\left(x_{0}+\xi\right)\right\}}{\lambda^{\nu+1}} d \lambda
\end{aligned}
$$




$$
-\frac{1}{2 \pi i} \int_{\mathbb{E}_{r}^{(+)}} \frac{e^{\lambda_{i}:}}{G(\lambda)} \sum_{k=0}^{n} \frac{1}{\lambda^{n-k}} \Lambda_{0}^{k}\left\{e^{\lambda \xi} \int_{x_{0}+\xi}^{x_{0}+\eta} e^{-\lambda \eta} f^{(n)}(\eta) d \eta\right\} d \lambda,
$$

where the first term of the right-hand side becomes

$$
\begin{aligned}
\frac{1}{2 \pi i} \int_{\substack{C_{r}(+)}} e^{\lambda x} & \left(\int_{x_{0}}^{x_{0}+b} e^{-\lambda \eta} f(\eta) d \eta\right) d \lambda \\
& =\frac{i}{2 \pi i} \int_{-\rho_{r}}^{\rho_{r}} e^{i \nu x}\left(\int_{x_{0}}^{x_{0}+b} e^{-i \nu \eta} f(\eta) d \eta\right) d \nu \\
& =\frac{1}{\pi} \int_{x_{0}}^{x_{0}+b} f(\eta) \frac{\sin \rho_{r}(x-\eta)}{x-\eta} d \eta,
\end{aligned}
$$

by means of the Cauchy's theorem.

Similarly we have

$$
\begin{aligned}
& \left.\mathrm{S}_{i} \cdot-\right)(x, x ; f) \\
& =\frac{1}{\pi} \int_{x_{0}+a}^{x_{0}} f(\eta) \frac{\sin \rho_{r}(x-\eta)}{x-\eta} d \eta
\end{aligned}
$$

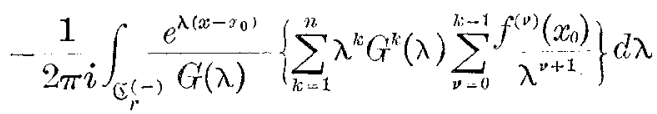

$$
\begin{aligned}
& +\frac{1}{2 \pi i} \int_{\mathfrak{C}_{r}^{(-)}} \frac{e^{\lambda\left(x-x_{0}-a\right)}}{G(\lambda)} \sum_{k=0}^{n} \lambda^{k} G_{k}(\lambda) \sum_{\nu=0}^{n-1} \frac{f^{\prime \nu)}\left(x_{n}+a\right)}{\lambda^{\nu+1}} d \lambda \\
& -\frac{1}{2 \pi i} \int_{\mathbb{C}_{\gamma^{\prime}-},} \frac{e^{\lambda\left(x-x_{0}\right)}}{G(\lambda)} \sum_{k=0}^{n-1} \sum_{\nu=0}^{n-k-1} \frac{\Lambda_{\xi}^{k}\left\{f^{(k+\nu)}\left(x_{0}+\xi\right)\right\}}{\lambda^{\nu+1}} d \lambda \\
& -\frac{1}{2 \pi i} \int_{\mathbb{E}_{r}^{t},-} \frac{e^{\lambda x}}{G(\lambda)} \sum_{k=0}^{n} \frac{1}{\lambda^{n-k}} \Lambda_{\xi}^{k}\left\{e^{\lambda \xi} \int_{v_{0}+\xi}^{x_{0}+a} e^{-\lambda \eta} f^{\prime n)}(\eta) d \eta\right\} d \lambda .
\end{aligned}
$$

Consequently, if we put

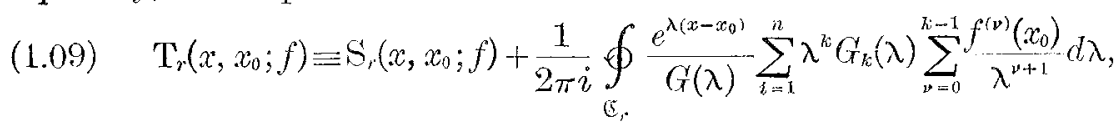

where the second term of the right hand side is the sum of the second terms of (1.06) and (1.08), then we have

(1.10) $\mathrm{T}_{r}\left(x, x_{0} ; f\right)$

$$
\begin{aligned}
= & \frac{1}{\pi} \int_{x_{0}+a}^{x_{0}+b} f(\eta) \frac{\sin \rho_{r} \cdot(x-\eta)}{x-\eta} d \lambda \\
& +\frac{1}{2 \pi i} \int_{\mathbb{C}_{r}^{(+)}} \frac{e^{\lambda\left(x-x_{0}-b\right)}}{\lambda} \sum_{\nu=0}^{n-1} \frac{f^{(\nu)}\left(x_{n}+b\right)}{\lambda^{\nu}} d \lambda
\end{aligned}
$$




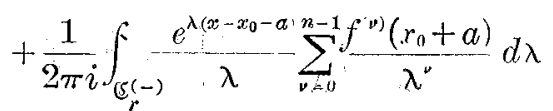

$$
\begin{aligned}
& -\frac{1}{2 \pi i} \oint_{\mathcal{E}_{5}} \frac{e^{\lambda\left(x-x_{0}\right)}}{G(\lambda)} \sum_{k=0}^{n-1} \sum_{\nu=0}^{n-k-1} \frac{\Lambda_{\xi}^{k}\left\{f^{(k+\nu)}\left(x_{0}+\xi\right)\right\}}{\lambda^{\nu-1}} d \lambda \\
& -\frac{1}{2 \pi i} \int_{\mathfrak{C}_{r}^{(+)}} \frac{e^{\lambda i v}}{G(\lambda)} \sum_{k=0}^{n} \frac{1}{\lambda^{n-\eta}} \Lambda_{\xi}^{k}\left\{e^{\lambda \varepsilon} \int_{j_{0}+\xi}^{x_{0}+\xi} e^{-\lambda \eta} f^{(n)}(\eta) d \eta\right\} d \lambda \\
& -\frac{1}{2 \pi i} \int_{\mathscr{C}_{r}^{(-)}} \frac{e^{\lambda x}}{G(\lambda)} \sum_{k=0}^{n} \frac{1}{\lambda^{n-k}} \Lambda_{0}^{k}\left\{e^{\lambda \xi} \int_{x_{0}+\xi}^{x_{0}+a} e^{-\lambda \eta} f^{(n)}(\eta) d \eta\right\} d \lambda,
\end{aligned}
$$

where the first term of the right-hand side in the Dirichlet's integral.

2. If $f(x)$ belongs to $\mathcal{L}_{n+m}\left(x_{0}+a, x_{i}+b\right)(m>0)$, then we have

$$
\begin{aligned}
& \sum_{k=0}^{n} \frac{1}{\lambda^{n-l}} \Lambda_{0}^{k}\left\{e^{\lambda \xi} \int_{x_{0}+\xi}^{x_{0}+b} e^{-\lambda \eta} f^{(n)}(\eta) d \eta\right\} \\
& =e^{-\lambda x_{0}} \sum_{r-0}^{n} \sum_{s=0}^{m-1} \frac{\Lambda_{0}^{k}\left\{f^{(n+s)}\left(x_{0}+\xi\right)\right\}}{\lambda^{n-k+s+1}} \\
& -e^{-\lambda\left(x_{0}+b\right)} \sum_{k=0}^{n} \lambda^{k} G_{k}(\lambda) \sum_{s=1}^{m-1} \frac{f^{n+s)}\left(x_{n}+b\right)}{\lambda^{n+s+1}} \\
& +\sum_{k=0}^{n} \frac{1}{\lambda^{n-k+m}} \cdot \Lambda_{\xi}^{k}\left\{e^{\lambda \xi} \int_{x_{0}+\xi}^{x_{0}+b} e^{-\lambda \eta} f^{(m+n)}(\eta) d \eta\right\},
\end{aligned}
$$

and consequently (1.10) becomes

$$
\begin{aligned}
& \text { (1.12) } \mathrm{T}_{r}\left(x, x_{0} ; f\right)
\end{aligned}
$$

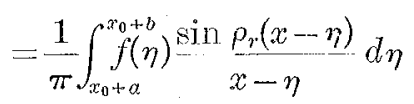

$$
\begin{aligned}
& +\frac{1}{2 \pi i} \int_{\mathfrak{C}_{r}^{(+)}} \frac{e^{\lambda\left(x-x_{0}-b\right)}}{\lambda} \sum_{\nu=0}^{n+m-1} \frac{f^{(\nu)}\left(x_{0}+b\right)}{\lambda^{\nu+1}} d \lambda \\
& +\frac{1}{2 \pi i} \int_{\mathbb{E}_{r}^{(-)}} \frac{e^{\lambda\left(x-x_{0}-a\right)} \lambda}{\lambda} \sum_{\nu=0}^{n+m-1} \frac{f^{(\nu)}\left(x_{n}+a\right)}{\lambda^{\nu+1}} d \lambda \\
& -\frac{1}{2 \pi i} \oint_{\mathbb{C}_{r}} \frac{e^{\lambda\left(x-x_{0}\right)}}{G(\lambda)} \tau_{n, m}\left(x_{0}, \lambda ; f\right) d \lambda \\
& -\frac{1}{2 \pi i} \int_{\mathfrak{C}_{r}^{(+)}} \frac{e^{\lambda x}}{G(\lambda)} \sum_{k=0}^{n} \frac{1}{\lambda^{n+m-k}} \Lambda_{0}^{k}\left\{e^{\lambda \xi} \int_{x_{0}+\xi}^{x_{0}+b} e^{-\lambda \eta} f^{(m+n)}(\eta) d \eta\right\} d \lambda \\
& -\frac{1}{2 \pi i} \int_{\mathfrak{G}(-)} \frac{e^{\lambda x}}{G(\lambda)} \sum_{k=1}^{n} \frac{1}{\lambda^{n+m-k}} \Lambda_{0}^{k}\left\{e^{\lambda \xi} \int_{x_{0}+\xi}^{x_{0}+a} e^{-\lambda \eta} f^{m+n)}(\eta) d \eta\right\} d \lambda \text {. }
\end{aligned}
$$


where, in order to simplify the integrand of the fourth term of the right hand side of (1.12), we have put for a moment

$$
\tau_{n, m}\left(x_{0}, \lambda ; f\right)=\sum_{k=0}^{n-1} \sum_{\nu=0}^{n-k:-1} \frac{a_{k+\nu}^{(k)}}{\lambda^{\nu+1}}+\sum_{k=0}^{n} \sum_{s=0}^{m-1} \frac{a_{s+n}^{(k)}}{\lambda^{n-k+s+1}},
$$

and

$$
a_{t}^{(k)}=\Lambda_{\xi}^{k}\left\{f^{(t)}\left(x_{0}+\xi\right)\right\}, \quad\left(\begin{array}{l}
k=0,1,2, \ldots, n \\
t=0,1,2, \ldots, n+m-1
\end{array}\right) .
$$

Then, by the rearrangement of the terms of (1.13), we have

$$
\begin{aligned}
\tau_{n, m}\left(x_{0}, \lambda ; f\right) & =\sum_{k=0}^{n-1} \sum_{\nu=1}^{n-k-1} \frac{a_{k+\nu}^{k)}}{\lambda^{\nu+1}}+\sum_{k=0}^{n-1} \sum_{s=0}^{m-1} \frac{a_{s+n}^{(k)}}{\lambda^{n-k+s+1}}+\sum_{s=0}^{m-1} \frac{a_{s+n}^{(n)}}{\lambda^{s+1}} \\
& =\sum_{k=0}^{n-1} \sum_{s=1}^{m-1} \frac{a_{s+k}^{(k)}}{\lambda^{s+1}}+\sum_{k=0}^{n-1} \sum_{s=m}^{n+m-k-1} \frac{a_{s+k}^{(k)}}{\lambda^{s+1}}+\sum_{s=0}^{m-1} \frac{a_{s++1}^{(n)}}{\lambda^{s+1}} \\
& =\sum_{k=0}^{n} \sum_{s=0}^{m-1} \frac{a_{s+k}^{(k)}}{\lambda^{s+1}}+\frac{1}{\lambda^{m}} \sum_{k=0}^{n-1} \sum_{s=0}^{n-k-1} \frac{a_{m+s+k}^{(k)}}{\lambda^{s+1}}
\end{aligned}
$$

Combining (1.14) and (1.15), we get

$$
\begin{aligned}
\tau_{n, m}\left(x_{\theta}, \lambda ; f\right)= & \sum_{k=0}^{n} \sum_{s=0}^{m-1} \frac{\Lambda_{\xi}^{k}\left\{f^{\prime s+k)}\left(x_{0}+\xi\right)\right\}}{\lambda^{s+1}} \\
& +\frac{1}{\lambda^{n}} \sum_{k=0}^{n-1} \sum_{s=0}^{n-k-1} \Lambda_{\xi}^{k}\left\{f^{(m+s+k)}\left(x_{0}+\xi\right)\right\}
\end{aligned}
$$

Substituting this in (1.12), it follows that

$$
\begin{aligned}
& \mathrm{T}_{r}\left(x, x_{0} ; f\right) \\
& =\frac{1}{\pi} \int_{r_{0}+a}^{x_{0}+b} f(\eta) \frac{\sin \rho_{r}(x-\eta)}{x-\eta} d \eta \\
& +\frac{1}{2 \pi i} \int_{\mathbb{E}_{r}^{(+)}} \frac{e^{\lambda\left(x-x_{0}-b\right)}}{\lambda} \sum_{\nu=0}^{n+m-1} \frac{f^{(\nu)}\left(x_{0}+b\right)}{\lambda^{\nu+1}} d \lambda \\
& +\frac{1}{2 \pi i} \int_{\tilde{E}_{r^{(-)}}^{(-)}} \frac{e^{\lambda\left(x-x_{0}-(a)\right.} G(\lambda)}{G(\lambda)} \sum_{\nu=0}^{n+m-1} \frac{f^{(\nu)}(x+a)}{\lambda^{\nu+1}} d \lambda
\end{aligned}
$$

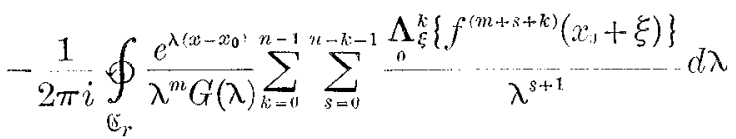

$$
\begin{aligned}
& -\frac{1}{2 \pi i} \int_{\mathfrak{E}_{r}^{(+)}} \frac{e^{\lambda x}}{\lambda^{m} G(\lambda)} \sum_{k=0}^{n} \frac{1}{\lambda^{n-k}} \Lambda_{n}^{k}\left\{e^{\lambda \xi} \int_{x_{0}+\xi}^{x_{0}+b} e^{-\lambda \eta} f^{(m+n)}(\eta) d \eta\right\} d \lambda \\
& \left.-\frac{1}{2 \pi i} \int_{\xi_{r}(-)} \frac{e^{\lambda x}}{\lambda^{m n} G(\lambda)} \sum_{k=0}^{n} \frac{1}{\lambda^{n-k}} \Lambda_{0}^{k}\left\{e^{\lambda \xi} \int_{x_{0}+\xi}^{x_{0}+a q} e^{-\lambda \eta} f^{(m+n)}(\eta) d \eta\right)\right\} d \lambda
\end{aligned}
$$




$$
-\frac{1}{2 \pi i} \oint_{\mathscr{C}_{r}} \frac{e^{\lambda\left(x-x_{0}\right)}}{G(\lambda)} \sum_{s=0}^{m-1} \frac{1}{\lambda^{s+1} \Gamma_{0}} \Gamma_{\xi}\left\{f^{(s)}\left(x_{0}+\xi\right)\right\} d \lambda .
$$

$3^{\circ}$. The results obtained for the sets $\mathscr{L}_{n+m}(m \geqq 0)$ may be so modified that we are able to deduce the formula suitable to more accurate estimations for the sets $\vartheta_{n+m}(m \geqq 0)$. For this, we remark that if $f(x)$ belongs to $V_{n+m}\left(x_{0}+a, x_{0}+b\right)(m \geqq 0)$, then we obtain, in stead of (1.11)

$$
\begin{aligned}
\sum_{k=0}^{n} \frac{-}{\lambda^{n-k}} \Lambda_{0}^{k}\left\{e^{\lambda \xi} \int_{x_{0}+\xi}^{\left.x_{0}+\hbar\right)} f^{(n)}(\eta) d \eta\right\} & \\
= & e^{-\lambda x_{0}} \sum_{i=0}^{n} \sum_{s=0}^{m} \frac{\Lambda_{\xi}^{k}\left\{f^{(n+s)}\left(x_{0}+\xi+0\right)\right\}}{\lambda^{n-k+s+1}} \\
& \left.-e^{-\lambda\left(x_{0}+b\right)} \sum_{k=0}^{n} \lambda^{k} G_{l c}(\lambda) \sum_{s=0}^{m} \frac{f^{(n+s)}\left(x_{0}+b-0\right)}{\lambda^{n+s+1}}-0\right) \\
& +\sum_{k=0}^{n} \frac{1}{\lambda^{n-k+m}} \Lambda_{0}^{k}\left\{e^{\lambda \xi} \int_{x_{0}+\xi}^{x_{0}+b} e^{-\lambda \eta} d f^{(m+n)}(\eta)\right\}
\end{aligned}
$$

and consequently, in stead of (1.17), we have

$$
\begin{aligned}
& \mathrm{T}_{r}\left(x, x_{0} ; f\right) \\
& =\frac{1}{\pi} \int_{x_{0}+a}^{x_{0}+b} f(\eta) \frac{\sin \rho_{r} \cdot(x-\eta)}{x-\eta} d \eta \\
& +\frac{1}{2 \pi i} \int_{\mathfrak{E}_{r}^{(+)}} \frac{e^{\lambda\left(x-x_{0}-b\right)}}{\lambda} \sum_{\nu=0}^{n+m} \frac{f^{(\nu)}\left(x_{0}+b-0\right)}{\lambda^{\nu+1}} d \lambda \\
& +\frac{1}{2 \pi i} \int_{\mathbb{E}_{r}^{(-)}} \frac{e^{\lambda\left(x-x_{0}-a\right)}}{\lambda} \sum_{\nu=0}^{n+m} \frac{f^{(\nu)}\left(x_{\theta}+a+0\right)}{\lambda^{\nu+1}} d \lambda \\
& -\frac{1}{2 \pi i} \int_{\mathcal{E}_{r}^{(+)}} \frac{e^{\lambda\left(x-x_{0}\right)}}{\lambda^{m+1} G(\lambda)} \sum_{k=0}^{n-1} \sum_{s=0}^{n-k-1} \frac{\Lambda_{\xi}^{k}\left\{f^{(m+s+k+1)}\left(x_{0}+\xi+0\right)\right\}}{\lambda^{s+1}} d \lambda \\
& -\frac{1}{2 \pi i} \int_{\mathbb{C}_{r}^{(-)}} \frac{e^{\lambda\left(x-x_{0}\right)} \lambda^{m+1} G(\lambda)}{\sum_{k=0}^{n-1 n-k-1}} \sum_{s=0}^{k} \frac{\Lambda_{\xi}^{k}\left\{f^{(m+s+k+1)}\left(x_{0}+\xi-0\right)\right\}}{\lambda^{s+1}} d \lambda \\
& -\frac{1}{2 \pi i} \int_{\mathbb{E}_{r}^{(+)}} \frac{e^{\lambda x}}{\lambda^{m+1} G(\lambda)} \sum_{k=0}^{n} \frac{1}{\lambda^{n-k}} \Lambda_{0}^{k}\left\{e^{\lambda \xi} \int_{x_{0}+\xi}^{x_{0}+b} e^{-\lambda \eta} d f^{(m+n)}(\eta)\right\} d \lambda \\
& -\frac{1}{2 \pi i} \int_{\mathbb{E}_{r}^{(-)}} \frac{e^{\lambda x}}{\lambda^{m+1} G(\lambda)} \sum_{k=0}^{n} \frac{1}{\lambda^{n-k}} \Lambda_{0}^{k \xi}\left\{e^{\lambda \xi} \int_{x_{0}+\xi}^{x_{0}+a} e^{-\lambda \eta} d f^{(m+n)}(\eta)\right\} d \lambda \\
& -\frac{1}{2 \pi i} \oint_{\mathfrak{E}_{1}} \frac{e^{\lambda\left(x-x_{0}\right)}}{G(\lambda)} \sum_{s=0}^{m} \frac{1}{\lambda^{s+1}} \Gamma\left\{f^{(s)}\left(x_{0}+\xi\right)\right\} d \lambda \text {. }
\end{aligned}
$$


Remark. Specially when $n=0$, that is, when the operator $\Gamma$ reduces to an operator $\Lambda$, then the formulae (1.10). (1.17) and $(1,19)$ become very simple. We will state these remarkable cases.

$1^{\circ}$. If $f(x)$ belongs to $\mathscr{L}_{0}\left(x_{0}+a, x_{0}+b\right)$, then we have

$$
\begin{aligned}
\mathrm{T}_{r}\left(x, x_{0} ; f\right)= & \mathrm{S}_{r}\left(x, x_{0} ; f\right) \\
= & \frac{1}{\pi} \int_{x_{0}+a}^{x_{0}+b} f(\eta) \frac{\sin \rho_{r}(x-\eta)^{2}}{x-\eta} d \eta \\
& +\frac{1}{2 \pi i} \int_{\mathcal{E}_{r}^{(+)}} G(\lambda) \Lambda_{\xi}^{\lambda x}\left\{e^{\lambda \xi} \int_{x_{0}+\xi}^{x_{0}+b} e^{-\lambda \eta} f(\eta) d \eta\right\} d \lambda \\
& -11 \int_{\mathcal{E}_{r}^{(-)}} \frac{e^{\lambda x}}{G(\lambda)} \Lambda_{\xi}\left\{e^{\lambda \xi} \int_{x_{0}+\xi}^{x_{0}+a} e^{-\lambda \eta} f(\eta) d \eta\right\} d \lambda .
\end{aligned}
$$

2 . If $f(x)$ belougs to $\mathcal{L}_{m}\left(x_{0}+a, x_{0}+b\right)$, then we have

$$
\begin{aligned}
& \mathrm{T}_{r}\left(x, x_{0} ; f\right)=\mathrm{S}_{r}\left(x, x_{0} ; f\right) \\
& =\frac{1}{\pi} \int_{x_{0}+a}^{x_{0}+b} f(\eta) \frac{\sin \rho_{r}(x-\eta)}{x-\eta} d \eta \\
& +\frac{1}{2 \pi i} \int_{\mathfrak{S}_{r}^{(+)}} \frac{e^{\lambda\left(x-x_{0}-b\right)}}{\lambda} \sum_{\nu=0}^{m-1} \frac{f^{(\nu)}\left(x_{n}+b\right)}{\lambda^{\nu}} d \lambda \\
& +\frac{1}{2 \pi i} \int_{\mathbb{E}^{(-)}} \frac{e^{\lambda\left(x-x_{0}-a\right)}}{\lambda} \sum_{\nu=0}^{m-1} f^{(\nu)} \frac{\left(x_{0}+a\right)}{\lambda^{\nu}} d \lambda \\
& -\frac{1}{2 \pi i} \int_{\mathbb{E}_{r}^{(+)}} \frac{e^{\lambda x}}{\lambda^{m} G(\lambda)} \Lambda_{0}\left\{e^{\lambda \xi} \int_{x_{0}+\xi}^{x_{0}+b} e^{-\lambda \eta} f^{(m)}(\eta) d \eta\right\} d \lambda \\
& -\frac{1}{2 \pi i} \int_{\left.\mathcal{E}_{r}-1\right)} \frac{e^{\lambda x}}{\lambda^{m}} G(\lambda) \Lambda_{\xi}\left\{e^{-\lambda \xi} \int_{x_{0}+\xi}^{x_{0}+a} e^{-\lambda \eta} f^{(m)}(\eta) d \eta\right\} d \lambda \\
& -\frac{1}{2 \pi i} \oint_{\mathbb{E}_{r}} \frac{e^{\lambda\left(x-x_{0}\right)}}{G(\lambda)} \sum_{s=0}^{n-1} \frac{1}{\lambda^{s+1}} \Lambda_{0}\left\{f^{(s)}\left(x_{0}+\xi\right)\right\} d \lambda .
\end{aligned}
$$

$3^{\prime}$ If $f(x)$ belongs $v_{0}\left(x_{0}+a, x_{0}+b\right)$, then, we have

(1.22) $\quad \mathrm{T}_{r}\left(x, x_{0} ; f\right)=\mathrm{S}_{r}\left(x, x_{0} ; f\right)$

$$
\begin{aligned}
= & \frac{1}{\pi} \int_{x_{0}+a}^{x_{0}+b} f(\eta) \frac{\sin \rho_{r} \cdot(x-\eta)}{x-\eta} d \eta \\
& +\frac{1}{2 \pi i} \int_{\left(\mathbb{E}_{r}(+)\right.} \frac{e^{\lambda\left(x-x_{0}-b\right)}}{\lambda} f\left(x_{0}+b-0\right) d \lambda \\
& +\frac{1}{2 \pi i} \int_{\mathbb{E}_{r}(-)} \frac{e^{\lambda\left(x-x_{0}-a\right)}}{\lambda} f\left(x_{0}+a+0\right) d \lambda
\end{aligned}
$$




$$
\begin{aligned}
& +\frac{1}{2 \pi i} \int_{\mathbb{E}_{r}^{(+)}} \frac{e^{\lambda x}}{\lambda G(\lambda)^{\alpha}} \mathbf{\Lambda}_{\xi}\left\{e^{\lambda \xi} \int_{x_{0}+\xi}^{x_{0}+b} e^{-\lambda \eta} d f(\eta)\right\} d \lambda \\
& -\frac{1}{2 \pi i} \int_{\mathcal{E}_{r}^{(-)}} \frac{e^{\lambda x}}{\lambda G(\lambda) \Lambda_{\xi}}\left\{e^{\lambda \xi} \int_{x_{0}+\xi}^{x_{0}+a} e^{-\lambda \eta} d f(\eta)\right\} d \lambda
\end{aligned}
$$

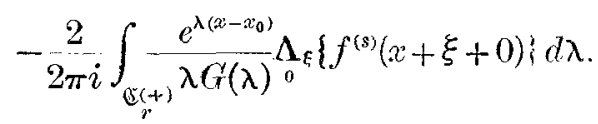

$$
\begin{aligned}
& -\frac{1}{2 \pi i} \int_{\sigma_{r}(-)} \frac{e^{\lambda\left(x-x_{0}\right)}}{\lambda G(\lambda)} \Lambda_{\xi}\left\{f^{(s)}\left(x_{0}+\xi-0\right)\right\} d \lambda .
\end{aligned}
$$

$4^{\circ}$ If $f(x)$ belongs to $V_{m}\left(x_{0}+a, x_{\theta}+b\right)$, then, we have

$$
\begin{aligned}
& \mathrm{T}_{r}\left(x, x_{0} ; f\right)=\mathrm{S}_{r}\left(x, x_{0} ; f\right) \\
& =\frac{1}{\pi} \int_{x_{0}+a}^{x_{0}+b} f(\eta) \frac{\sin \rho_{r}(x-\eta)}{x-\eta} d \eta \\
& +\frac{1}{2 \pi i} \int_{\mathbb{C}_{r}^{(+)}} \frac{e^{\lambda\left(x-x_{0}-b\right)}}{G(\lambda)} \sum_{v=0}^{m} \frac{f^{(v)}\left(x_{0}+b-0\right)}{\lambda^{\nu}} d \lambda \\
& +\frac{1}{2 \pi i} \int_{\mathbb{Q}^{(-)}} \frac{e^{\lambda\left(x-x_{0}-a\right)}}{\lambda} \sum_{\nu=0}^{m} \frac{f^{(\nu)}\left(x_{0}+a+0\right)}{\lambda^{\nu}} d \lambda \\
& -\frac{1}{2 \pi i} \int_{\left.\sigma_{(}^{(+)}\right)} \frac{e^{\lambda x}}{\lambda^{m+1} G(\lambda)} \Lambda_{\xi}\left\{e^{\lambda \xi} \int_{x_{0}+\xi}^{x_{0}+b} e^{-\lambda \eta} d f^{(m)}(\eta)\right\} d \lambda \\
& -\frac{1}{2 \pi i} \int_{\mathbb{E}_{(-)}(-)} \frac{e^{\lambda x}}{\lambda^{m+1} G(\lambda)} \Lambda_{\xi}\left\{e^{\lambda \xi} \int_{x 0+\xi}^{x_{0}+a} e^{-\lambda \eta} d f^{(m)}(\eta)\right\} d \lambda \\
& -\frac{1}{2 \pi i} \int_{\mathbb{E}_{r}^{(+)}} \frac{e^{\lambda\left(x-x_{0}\right)}}{G(\lambda)} \sum_{s=0}^{m} \frac{1}{\lambda^{s+1}} \Lambda_{n}\left\{f^{(s)}\left(x_{0}+\xi+0\right)\right\} d \lambda \\
& -\frac{1}{2 \pi i} \int_{\mathbb{C}_{r}^{(-)}} \frac{e^{\lambda\left(x-x_{0}\right)}}{G(\lambda)} \sum_{s=0}^{n} \frac{1}{\lambda^{s+1}} \Lambda_{n}\left\{f^{i s)}\left(x_{0}+\xi-0\right)\right\} d \lambda
\end{aligned}
$$

\section{§2. Preliminary Considerations.}

Now we will state a method of estimating the second and the following terms of the right-hand sides of (1.10), (1.17) and (1.19) defined for a sequence of contour: $\left\{\mathfrak{S}_{r}\right\}$.

1. Estimation of the and and 3id integrals in (1.10), (1.1\%) and (1.19).

The integrals ${ }^{(99)}$

(9) Here $k$ is a fixed integer independent of $r$. 


$$
\int_{\mathfrak{E}_{\nu^{(+)}}^{(+)}} \frac{e^{\lambda\left(x-x_{0}-b\right)}}{\lambda} \sum_{\nu=0}^{k} \frac{f^{(\nu)}\left(x_{1)}+b\right)}{\lambda^{\nu}} d \lambda
$$

and

$$
\int_{\mathscr{C}_{r}^{(-)}} \frac{e^{\lambda\left(x-\nu_{0} \cdots(a)\right.}}{\lambda} \sum_{\nu=0}^{k_{i}} \frac{f^{(\nu)}\left(x_{0}+a\right)}{\lambda^{\nu}} d \lambda
$$

tend to zeros as $r \rightarrow \infty$, uniformly in the interval $\left(x_{0}+a+\delta_{1}, x_{0}+b-\delta_{2}\right)$, if we assume the following two conditions concerning the bchaviours of the contours $\left\{\mathbb{E}_{i}\right\}$ :

Condition $1^{\circ}$. The distances $d_{r}$ between the contour $\mathbb{E}_{r}$ and the origin tends to infinity as $r \rightarrow \infty$.

\section{Condition $2^{\circ}$.}

$$
\int_{\mathbb{C}_{r}^{(+)}}\left|e^{-\lambda \delta z}\right||d \lambda|=O(1)
$$

for a certain $\delta_{2}>0$, and

$$
\int_{\mathbb{C}_{r}^{(-)}}\left|e^{\lambda \delta_{1}}\right||d \lambda|=O(1)
$$

for a certain $\delta_{1}>0$, a,s $r \rightarrow \infty$.

2. Estimation of the fth and the following integrals in (1.10), (11\%) and (1.19) except the last members of (1.17) and (1.19).

It is sufficient to estimate the following integrals:

$$
\int_{\mathcal{C}_{i}(\neq)} \frac{e^{\lambda\left(x-x_{0}\right)}}{\lambda^{m} G(\lambda)}|d \lambda|
$$

and

$$
\int_{\mathbb{E}_{r}^{(t)}} \frac{e^{\lambda x}}{\lambda^{s} G(\lambda)} \Lambda_{\xi} e^{i \xi} \int_{x_{0}+\xi}^{x_{0}+\gamma} e^{-\lambda \eta} f^{(t)}(\eta) d \eta|d \lambda|,
$$

where $y=b$ for $\mathfrak{S}_{r}^{(+)}$, and $\gamma=a$ for $\mathfrak{E}_{r}^{(-)}$.

We will show that the estimations of (2.06) may be reduced to those of (2.05). For this purpose we will remind three lemmas ${ }^{(30)}$ :

Lemma 1. If $f(t)$ is integrable on the finite interval $(p, q)$ and we write $\lambda=\rho e^{i \theta}$, then we have, when $|\lambda|=\rho \rightarrow \infty$,

${ }^{\left({ }^{30}\right)}$ Proofs of the first two lemmas are given in Titchmarsh [1] and Tamarkin [1]. See Titchmarsh [1] 2.2. Lemma 2.2. p. 288. and Tamarkin [1] p. 30. Lemma. The Proof of the third lemma is given in R. C. Young [1], § \& The asymptotic behaviour of $F(z)\left(=\int_{a-0}^{b+a} e^{\lambda t} d \varphi(t)\right)$. Path of determination. p. 308. The arguments of the proofs are necessary in the following. 


$$
\begin{aligned}
\int_{p}^{q} e^{\lambda t} f(t) d t & =o\left(e^{\rho t \cos \theta}\right), & & \text { for }-\frac{\pi}{2} \leqq \theta \leqq \frac{\pi}{2} . \\
& =o\left(e^{\rho p \cos \theta}\right), & & \text { for } \quad \frac{\pi}{2} \leqq \theta \leqq \frac{3 \pi}{2} .
\end{aligned}
$$

Lemma 2. If $f(t)$ is of bounded cariation on the finite interval $(p, q)$,

$$
\int_{p}^{q} e^{\lambda t} f(t) d t=\frac{A_{1}(\lambda)}{\lambda} e^{\lambda q}+\frac{A_{2}(\lambda)}{\lambda} e^{\lambda p}
$$

where $A_{i}(\lambda)(i=1,2)$ are bounded for large values of $|\lambda|$.

Lemma 3. If $f(t)$ is of bounded variation on the finite interval ( $p$, $q)$, and is continuous at the endpoints $p$ and $q$, then we have

$$
\begin{aligned}
\int_{i p}^{q} e^{\lambda t} d f(t) & =o\left(e^{p \gamma \cos \theta}\right), & & \text { for }-\frac{\pi}{2} \leqq \theta \leqq{ }_{2}^{\pi} \\
& =o\left(e^{\rho q \cos \theta}\right), & & \text { for } \quad \frac{\pi}{2} \leqq \theta \leqq \frac{3 \pi}{2} .
\end{aligned}
$$

We will now distinguish two cases:

Case I. Let $f(x)$ be a function of $\mathcal{L}_{t}\left(x_{0}+a, x_{0}+b+\eta\right) . \quad(\eta>0)$ Then, by Lemma 1 and by the first part of Property III of $\Lambda$ for $R(\lambda) \geqq 0$,

$$
\Lambda_{0}\left\{e^{\lambda \xi} \int_{x_{0}+\xi}^{x_{0}+b} e^{-\lambda \eta} f^{(t)}(\eta) d \eta\right\}=\int_{0}^{\delta} e^{-\lambda \eta} \Lambda f^{(t)}\left(x_{0}+\eta\right) d \eta+\Lambda_{\xi}\left[e^{\lambda \xi} \int_{x_{0}+\xi+\delta}^{x_{0}+\eta} e^{-\lambda \eta} f^{(t)}(\eta) d \eta\right]
$$

and therefore, by the Titchmarsh's argument and Property $\operatorname{III}\left({ }^{31}\right)$,

$$
\Lambda_{0}\left\{e^{\lambda \xi} \int_{x_{0}+\xi}^{x_{0}+t} e^{-\lambda \eta} f^{(t)}(\eta) d \eta\right\}=o\left(\left|e^{-\lambda x_{0}}\right|\right), \quad \text { for } R(\lambda) \geqq 0 .
$$

Here the auxiliary prolongation of $\mathscr{L}_{t}\left(x_{0}+a, x_{0}+b\right)$ to $\mathscr{L}_{t}\left(x_{0}+a, x_{0}+b+\right.$ $\eta)$ may be easily done. Similarly for $R(\lambda) \leqq 0$. Hence, as $r \rightarrow \infty$

$$
\int_{\mathbb{E}_{,}( \pm)} \frac{e^{\lambda x}}{\lambda^{s} G(\lambda)} \Lambda_{\nu^{\prime}}\left\{e^{\lambda \xi} \int_{x_{0}+\xi}^{x_{0}+\gamma} e^{-{ }^{-\lambda \eta}} f^{(t)}(\eta) d \eta\right\}|d \lambda|=o\left(\int_{\mathbb{C}_{r}^{(t)}} \frac{e^{\lambda\left(x-x_{0}\right)}}{\lambda^{s} G(\lambda)}|d \lambda|\right) .
$$

Case II. Let $f(x)$ be a function which belongs to $v_{t}\left(x_{0}+a, x_{0}+b\right)$. Then, by Lemma 2 and Property III of $\Lambda$,

$$
\begin{array}{ll}
\Lambda_{\xi}\left\{e^{\lambda \xi} \int_{x_{0}+\xi}^{x_{0}+b} e^{-\lambda \eta} f^{(t)}(\eta) d \eta\right\}=\frac{A(\lambda)}{\lambda} e^{-\lambda x_{0}}, & \text { for } R(\lambda) \geqq 0, \\
\Lambda_{\xi}\left\{e^{\lambda \xi} \int_{x_{0}+\xi}^{x_{0}+a} e^{-\lambda \eta} f^{(t)}(\eta) d \eta\right\}=\frac{B(\lambda)}{\lambda} e^{-\lambda x_{0}}, & \text { for } R(\lambda) \leqq 0,
\end{array}
$$

(31) See Titchmarsh [1] p. $\longleftarrow 88$. (Proof of Lemma 2.2.) Here we notice that, for every $a \leqq \xi \leqq b$, we have, for $0 \leqq \delta<\eta$,

$$
\left\{\begin{array}{l}
1^{\circ} \cdot\left|\int_{0}^{\delta} e^{-\lambda \eta} \Lambda f^{(t)}\left(x_{0}+\eta\right) d \eta\right| \leqq \int_{0}^{\delta}\left|\Lambda f^{(t)}\left(x_{0}+\eta\right)\right||d \eta|, \text { which tends to zero, as } \delta \rightarrow 0 . \\
2^{\circ} \cdot\left|e^{\lambda \xi} \int_{x_{0}+\xi+\delta}^{x_{0}+\nu} e^{-\lambda \eta} f(t)(\eta) d \eta\right| \leqq\left|e^{-\lambda\left(x_{0}+\delta\right) \mid}\right| \int_{a}^{b+\delta}\left|f^{(t)}\left(x_{0}+\eta\right)\right| ! d \eta \mid, \text { which tends to zero uniformly in } \xi
\end{array}\right.
$$

Here $\lambda$ is subject to $R(\lambda) \geqq 0$. Therefore we can apply the latter yart of Property III to $3^{\circ}$. 
where $A(\lambda)$ and $B(\lambda)$ are bounded for large values of $|\lambda|$ in the right and left half-planes, respectively.

Therefore we reach the result

$$
\begin{aligned}
\int_{\mathbb{E}_{,}^{( \pm)}} \frac{e^{\lambda x}}{\lambda^{s} G(\lambda)} \|\left|\Lambda_{\xi}\left\{e^{\lambda \xi} \int_{x_{0}+\xi}^{x_{0}+\gamma} e^{-\lambda \eta} f^{(t)}(\eta) d \eta\right\}\right| d \lambda \mid & \\
= & O\left(\int_{\mathbb{E}_{r}^{( \pm)}} \frac{e^{\lambda\left(x-x_{0}\right)}}{\lambda^{s+1} G(\lambda)}|| d \lambda \mid\right), \quad(\text { as } r \rightarrow \infty) .
\end{aligned}
$$

Case III. Let $f(x)$ be a function which belongs to $V_{t}(x+a, x+b)$. Then there is another estimation which appeals to Lemma 3 and Property III and which yields us that, for $|R(\lambda)| \leqq \frac{\pi}{2}$,

$$
\begin{aligned}
& \Lambda_{0}\left\{e^{\lambda \xi} \int_{x_{0}+\xi}^{x_{0}+b} e^{+\lambda \eta} f^{(t)}(\eta) d \eta\right\} \\
& =\frac{e^{-\lambda x_{0}}}{\lambda} \Lambda_{0}\left\{f^{(t)}\left(x_{0}+\xi+0\right)\right\}-\frac{e^{-\lambda\left(x_{0}+b\right)}}{\lambda} G(\lambda) f^{(t)}\left(x_{0}+b-0\right) \\
& \quad+o\left(\mid \frac{e^{-\lambda x_{0}}}{\lambda}\right)
\end{aligned}
$$

and that, for $-\frac{\pi}{2} \leqq R(\lambda) \leqq \frac{3 \pi}{2}$,

$$
\begin{aligned}
& \Lambda_{\xi}\left\{e^{\lambda \xi} \int_{0+\xi}^{x_{0}+a} e^{-\lambda \eta} f^{()}(\eta) d \eta\right\} \\
& =\frac{e^{-\lambda x_{0}}}{\lambda} \Lambda_{0}\left\{f^{(t)}\left(x_{0}+\xi-0\right)\right\}-\frac{e^{-\lambda\left(x_{0}+a\right)}}{\lambda} f^{(t)}\left(x_{0}+a+0\right) G(\lambda) \\
& \quad+o\left(\frac{e^{-\lambda x_{0}}}{\lambda}\right)
\end{aligned}
$$

as $|\lambda|=\rho \rightarrow \infty$.

\section{Convergence-theorems of Cauchy's Series.}

We will begin with some definitions.

Definition III. A sequence of contours $\left\{\mathfrak{E}_{r}\right\}$ defined in Introduction is said to be associated to the interval $\left(\delta_{1},-\delta_{2}\right)$ if $\mathfrak{S}_{r}$ satisfies Conditions $1^{\circ}$ and $\mathscr{P}^{\circ}$ introduced in $\$ 2.1$, and then we write

$$
\left\{\mathfrak{S}_{r}\right\} \in \mathbb{E}\left(\delta_{1},-\delta_{2}\right) \text {. }
$$

Definition IV. An integral function $G(\lambda)$ is said to be $O$-or o-associated to the sequence of contours $\left\{\mathfrak{E}_{2}\right\}$ with the index $s$ in the inter'al $\left(a+\delta_{1}{ }^{\prime}, b-\delta_{2}{ }^{\prime}\right)$, if $G(\lambda)$ has no zero-points on any contours of this sequence, and further if

$$
\int_{\mathfrak{C}_{r}^{( \pm)}} \frac{e^{\lambda q}}{\mid \lambda^{s} G(\lambda)}|d \lambda|=O(1), \quad(\text { as } r \rightarrow \infty),
$$


(3.03)

$$
\int_{\left(S_{r}( \pm)\right.}\left|\frac{e^{\lambda q}}{\lambda^{s} G(\lambda)}\right| d \lambda \mid=o(1), \quad(\text { as } r \rightarrow \infty),
$$

uniformly concerning $q$ in the interval $\left(a+\delta_{1}{ }^{\prime}, b-\delta_{2}{ }^{\prime}\right)$ respectively, and then we write

$$
G(\lambda)=\mathfrak{C}_{r}-O\left(s ; a+\delta_{1}{ }^{\prime}, b-\delta_{2}{ }^{\prime}\right),
$$

or

$$
G(\lambda)=\mathcal{C}_{r}-o\left(s ; a+\delta_{t}^{\prime}, b-\delta_{2}^{\prime}\right),
$$

respectively.

Definition V. A function $f(x)$, defined in the interval $\left(x_{0}+a, x_{n}+b\right)$, is said to satisfy the boundary condition of the s-th order with respect to the operator $\Gamma$ at the point $x_{0}$, if we have

$$
\Gamma_{\xi}\left\{f^{(k)}\left(x_{0}+\xi\right)\right\}=0, \quad(k=0,1,2, \ldots, s-1)
$$

where $s$ is a positive integer, and we then write

$$
B_{s}\left(f ; x_{0}\right)=0 .
$$

Using these terminologies the convergence-theorem, which we are going to prove, may be stated as follows:

Theorem III. I. Let $f(x)$ be a function which bolongs to $\left(\mathcal{L}_{n+m}\right)$ and which satisfies the boundary condition ${ }^{(33)}$

$$
B_{m}\left(f ; x_{m}\right)=0 .
$$

Let it be assumed that there is a sequence of contours such that

$$
\left\{\mathbb{E}_{r}\right\} \varepsilon \in\left(\delta_{1},-\delta_{2}\right) \text {, }
$$

and further that, with respect to this sequence,

$$
G(\lambda)=\mathbb{E}_{r}-O\left(m ; a+\delta_{1}{ }^{\prime}, b-\delta_{2}{ }^{\prime}\right) .
$$

Then the contour-integrals $\left\{\mathrm{T}_{r}\left(x, \hat{x}_{0} ; f\right)\right\}$ defined for this sequence $\left\{\mathfrak{C}_{r}\right\}$ tend to the Dirichlet's integral uniformly concerning $x$ in the interval $\left(x_{0}+a+\delta_{1}^{\prime \prime}, x_{0}+b-\delta_{2}^{\prime \prime}\right)$, where

and

$$
\delta_{1}^{\prime \prime}=\operatorname{Max}\left(\delta_{1}, \delta_{1}^{\prime}\right)
$$

$$
\delta_{2}^{\prime \prime}=\operatorname{Max}\left(\delta_{2}, \delta_{2}^{\prime}\right)
$$

as $r$ tends to infinity; that is to say, if we write

$$
\mathrm{T}_{r}\left(x, x_{i} ; f\right)=\frac{1}{\pi} \int_{x_{0}+a}^{x_{0}+b} f(\eta) \frac{\sin \rho_{r}(x-\eta)}{x-\eta} d \eta+\varepsilon_{r}\left(x, x_{0} ; f\right),
$$

then we have

$$
\lim _{r \rightarrow \infty} \varepsilon_{r}\left(x, x_{*} ; f\right)=0,
$$

uniformly concerning $x$ in the interval $\left(x_{0}+a+\delta_{1}^{\prime \prime}, x_{0}+b-\delta_{2}^{\prime \prime}\right)$ where $\delta_{1}^{\prime \prime}$ and $\delta_{2}^{\prime \prime}$ are subject to the conditions (3.11).

(3i) Here $m$ is a, non-negative integer, For $m=0$, we assume no boundary condition. 
Except when $n+m=0$, we are able to conclude that this Dirichlet's integral tends to $f(x)$ uniformly in $\left(x_{0}+a+\delta_{1}^{\prime \prime}+\eta, x_{0}+b-\delta_{2}^{\prime \prime}-\eta\right)$ as $r \rightarrow \infty$, $\eta$ being any given positive number.

Theorem III. II. Let $f(x)$ be a function which belongs to $\left(V_{n+m}\right)$ and satisfies the boundary condition (3.08). Then in the hypothesis of Theorem III.I the assumption (3.10) may be replaced by the weaker assumption that

$$
G(\lambda)=\mathfrak{C}_{r}-o\left(m+1 ; a+\delta_{1}^{\prime \prime}, b-\delta_{2}^{\prime \prime}\right),
$$

and the conclusion of Theorem III.I. remains unchanged.

To prove these theorems, let us assume first $m=0$. In this case we remind the formula (1.10), and then apply the considerations in $\$ 2$. Thus by the assumption (3.09) the second and the third integrals of the righthand side of (1.10) tend to zeros as $r \rightarrow \infty$, uniformly concerning $x$ in the interval $\left(x_{1}+a+\delta_{1}, x_{0}+b-\delta_{2}\right)$. Further the fourth and the following integrals of the right-hand side of (1.10) tend to zeros ais $r \rightarrow \infty$, uniformly concerning $x$ in the interval $\left(x_{0}+a+\delta_{1}{ }^{\prime}, x_{1}+b-\delta_{2}{ }^{\prime}\right)$, which is assured by the assumptions (3.10) for $\left(\mathscr{L}_{n+m}\right)$ and by $(3.14)$ for $\left(V_{n+m}\right)$ respectively. Therefore the contour-integrals $\left\{\mathrm{T}_{r}\left(x, x_{0} ; f\right)\right\}$ defined for the sequence of contours $\left\{\mathbb{C}_{r}\right\}$ tend to the Dirichlet's integral, the first term of the righthand side of (3.10). The remains of the conclusions in both theorems may be obtained as a well-known property of Dirichlet's integral.

Next let $m \geqq 1$. Then we remind the formula (1.18). We may dispose with the integrals of the right-hand side of (1.18) quite similarly as in the former case $m=0$ except the last one

$$
-\frac{1}{2 \pi i} \oint_{\mathfrak{C}_{r}} \frac{e^{\lambda\left(x-x_{0}\right)}}{G(\lambda)} \sum_{s=0}^{m-1} \frac{1}{\lambda^{s+1}} \Gamma_{0}\left\{f^{(s)}\left(x_{0}+\xi\right)\right\} d \lambda,
$$

which, however, is equal to zero by the boundary condition (3.08).

Thus we have completely proved our theorems.

In view of (1.19), (2.16) and (2.17), we are able to prove, by similar method, the following

Theorem III. III. Let $f(x)$ be a function which belongs to $\left(\vartheta_{n+m}\right)$ and which satisfies the boundary conditions

$$
B_{m+1}\left(f ; x_{11}+0\right)=0
$$

and

$$
B_{m+1}\left(f ; x_{0}-0\right)=0 .
$$

Then in the hypothesis of Theorem III. II. the assumption (3.1.4) may be replaced by

$$
G(\lambda)=\mathfrak{E}_{r}-O\left(m+1 ; a+\delta_{1}^{\prime \prime}, b-\delta_{2}\right)
$$

and the conclusion of Theorem III. II. remains true. 


\section{$\S 4$. Asymptotic Behaviour of an Integral Function and Conditions of Convergence in $\S 3$.}

We will prove the following theorem, which gives the cxamples of inte. gral functions satisfying the convergence-conditions stated in $\$ 3$, and which. reads as follows:

Theorem IV. I. Let an integral function $G(\lambda)$ be expressed, for a certain integer $m$, by the asymptotic representation

$$
\lambda^{m} G(\lambda)=\sum_{i=0}^{s} \lambda^{v_{i}} A_{i}(\lambda) e^{\lambda h_{i}}, \quad(m>0)
$$

where

and

$$
\begin{cases}1^{\circ} . & \nu_{0} \geqq 1, \quad v_{n} \geqq 1 . \\ 2^{\circ} . & a_{0} a_{s} \neq 0 . \\ 3^{\circ} . & \text { The coefficients } A_{i}(\lambda) \text { are of the forms }\end{cases}
$$

$$
A_{i}(\lambda)=a_{i}+\epsilon_{i}(\lambda)
$$

$a_{i}$ being constants and $\epsilon_{i}(\lambda)$ tending to zeros as $|\lambda| \rightarrow \infty,(i=0,1, \ldots, s)$.

Then there is a sequence of contours $\left\{\mathfrak{C}_{,}\right\}$associated to the interval $(\varepsilon,-\varepsilon)$, to which $G(\lambda)$ is o-associated with the index $m$ in the interval $\left(h_{0}+\varepsilon, h_{s}-\varepsilon\right)$, $\varepsilon$ being any given positive number.

To prove this, we proceed in three steps.

As the first step we will quote the results due to Langer [1] which gives the assertions concerning the zero-points of exponential sums of the form (4.01). As the next step, we will construct a sequence of contours, using the results of Langer's. Lastly, we will finish our proof in showing that the sequence of contours there constructed has the desired properties in Theorem IV. I.

1. Langer's results on zeros of exponential sums. $\left({ }^{34}\right)$ There are two cases to be considered, of which the first caso is a special and pr liminary one of the second:

Case I. Preliminary Case. In this case we suppose that $\nu_{i}$ and $h_{i}$ are proportional, that is, $\nu_{i}=h_{i} \beta(i=0,1,2, \ldots, s)$, then the zeros of $\lambda^{m} G(\lambda)$ are asymptotically located within a logarithmic curvilinear strip bounded by the curves

$$
\xi+\beta \log |\lambda|= \pm K
$$

where we write $\lambda=\xi+i \eta$, and $K$ is suitably chosen constant.

By $n\left(y_{1}, y_{2}\right)$ we denote the number of zeros of $\lambda^{m} G(\lambda)$ lying between any two given parallel lines $I(\lambda)=y_{1}$ and $I(\lambda)=y_{2},\left(y_{2}>y_{1}\right)$ then we have

(31) Langer [I], Part I. 6, The Values $v_{j}$ and $c_{j}$ Proportional. (p. 221-292). 


$$
-s+\frac{h_{s}-h_{0}}{2 \pi}\left(y_{2}-y_{1}\right) \leqq n\left(y_{1}, y_{2}\right) \leqq s+\frac{h_{s}-h_{0}}{2 \pi}\left(y_{2}-y_{1}\right)
$$

For any given sufficiently small positive number $\delta$ there exists a constant $H_{\delta}$ depending only on $\delta$ and $G(\lambda)$ and such that

$$
\left|\lambda^{m} G(\lambda)\right|>H_{\delta}, \quad \text { for } \quad\left|\lambda-\lambda_{k}^{*}\right|<\delta,
$$

where $\lambda_{k}^{*}$ is any zero-point of $\lambda^{m} G(\lambda)$.

In this case we say that $\lambda$ is uniformly bounded form the zeros of $\lambda^{m} G(\lambda)$.

\section{Case II, General Case. $\left({ }^{35}\right)$}

In this case we do not suppose the proportionality of $\nu_{i}$ and $h_{i}$.

After Langer's construction, we are able to associate in $\lambda$-plane the points $P_{j}$ with Cartesian coordinates $\left(h_{j}, \nu_{j}\right)(j=0,1,2, \ldots, s)$.

These points determine, uniquely, the broken (or straight) line $L$, with the following properties:

$$
\begin{cases}1^{\circ} & L \text { joins } P_{0} \text { with } P_{s} . \\ 2 .^{\circ} & L \text { has vertices only at the points } P_{j .} \\ 3 .^{\circ} & L \text { is convex upward (or straight). } \\ 4 .^{\circ} & \text { No points of }\left\{P_{j}\right\} \text { lie above } L . \quad \text { (See Fig. 2). }\end{cases}
$$

By the suitable change of suffices, we can suppose that $P_{j}(j=0,1, \ldots$, $l$ ) are vertices. (When $L$ is straight, then $l=1$, and vice versa).

Let $L_{r}$ designate the $r$-th segment of this broken line $L_{1}$, the order being from the left to the right, and let the slope of $L_{r}$ be denoted by $m_{r}$. Then we have

$$
\left\{\begin{array}{l}
1 .{ }^{\circ} \quad m_{0}>m_{1}>m_{2}>\cdots>m_{l-1}, \quad(l>1) \cdot\left({ }^{36}\right) \\
2 .^{\circ} \text { there is one and only one integer } k \text { such that }
\end{array}\right.
$$

$$
m_{k-1} \geqq 0>m_{k}, \quad(l>1) \cdot\left({ }^{37}\right)
$$

Then, for all $\lambda$ in the region bounded by the curves

$$
\left\{\begin{array}{l}
\xi=-\left(m_{r-1}+\epsilon\right) \log |\lambda|, \\
\xi=-\left(m_{r}-\epsilon\right) \log |\lambda|
\end{array}\right.
$$

$\epsilon$ being positive and sufficiently small but otherwise arbitrary, $\lambda^{m} G(\lambda)$ has the asymptotic representation

$$
\lambda^{m} G(\lambda)=\sum_{n-1}^{n_{r}} \lambda^{r_{r}{ }^{h}} A_{r, h}(\lambda) e^{c_{r, h}}
$$

( $\left.{ }^{35}\right)$ Langer [I]. Part I. 7. General Real Values $v_{j}$. (p. $\left.322-224\right)$.

(36) When $l=1$, then $m_{0}=m_{l-1} \supseteqq 0$.

(37) We put $K=\operatorname{Max}_{\substack{n \leq i \leq l-1}}\left\{K_{i}\right\}$. 
where the coefficients $A_{r, h}(\lambda)$ are of the forms

$$
A_{r, h}(\lambda)=a_{r_{,} h}+\epsilon_{r, h}(\lambda), \quad\left(a_{r, n_{r}} a_{r, 1} \neq 0\right) .
$$

Let $P_{j}(j=0,1,2, \ldots, l)$ be the points with $c_{j 1}$ as abscissa and $\nu_{j, 1}$ as rdinates.

Then we have

$$
P_{j}=\left(c_{j, 1}, v_{j, 1}\right)=\left(c_{j-1}, v_{j-1, n_{j-1}}\right), \quad(j=1,2, \ldots, l),
$$

for

$$
c_{j, 1}=c_{j-1}, n_{j-1},
$$

and

$$
\nu_{j, 1}=\nu_{j-1}, n_{j-1}, \quad(j=1,2, \ldots, l) .
$$

Now as (4.09) takes the form of the preceeding case, we get the following conclusions:

1. Zeros of $\lambda^{m} G(\lambda)$ in the region (4.09) are asymptotically confined in the logarithmic strip bounded by the curves

$$
\xi+m_{r} \log |\lambda|= \pm K_{r}
$$

for suitably chosen positive constants $K_{r}(r=0,1,2, \ldots, l-1)$.

2. $\mathrm{By} n\left(y_{1}, y_{2}\right)$ we denote the number of zeros lying between any two parallel lines $I(\lambda)=y_{1}$, and $I(\lambda)=y_{2},\left(y_{2}<y_{1}\right)$,

then we have

$$
-n_{r}+\frac{c_{r}, n_{r}-c_{r, 1}}{2 \pi}\left(y_{2}-y_{1}\right) \leqq n\left(y_{1}, y_{2}\right) \leqq n_{r}+\frac{c_{r}, n_{r}-c_{n_{r}, 1}}{2 \pi}\left(y_{2}-y_{1}\right)
$$

3. If $\lambda$ is uniformly bounded from the zoro-points of $\lambda^{m} G(\lambda)$ in the region (4.09), then there exist positive numbers $K_{\delta}$ and $H_{\delta}$ depending only on. $\delta$ and $G(\lambda)$ such that

and

$$
\left|\lambda^{m} G(\lambda)\right| \geqq K_{\delta}\left|\lambda^{v, r}, e^{c_{r}, 1 \lambda}\right|
$$

$$
\left|\lambda^{m} G(\lambda)\right| \geqq H_{\delta}\left|\lambda^{n-1,1} e^{c} n-1,1\right|,
$$

for $\lambda$ belonging to the region (4.09) and

such that

$$
\left|\lambda-\lambda_{k}^{*}\right|<\delta
$$

where $\delta$ is any zelo point of $\lambda^{n} G(\lambda)$.

2. Construction of a sequence of contour $\left\{\mathfrak{E}_{n}\right\}$.

By these results due to Langer we will now construct a sequence of contours. For this purpose let us separate the region $\left(S_{I I}^{+}\right)$defined by $I(\lambda) \geqq 0$ and $|\lambda| \geqq M$ into the set of domains $\left(D_{0}^{+}\right)+\left(D_{1}^{+}\right)+\ldots+\left(D_{l}^{+}\right)$as follows $\left.{ }^{39}\right)$ :

1. ${ }^{\circ}\left(D_{0}^{+}\right)$is the part of $\left(S_{M}^{*}\right)$ which is bounded by

$$
\xi+m_{0} \log |\lambda|=-k
$$

and the negative axis of reals.

(38) See Fig. 1. in the following. 
2. ${ }^{\circ}\left(D_{i}^{+}\right)$is the part of $\left(S_{M}^{+}\right)$which is bounded by

$$
\xi+m_{l-1} \log |\lambda|=K,
$$

and the positive axis of reals.

$3 .^{\circ}\left(D_{1}^{+\infty}\right)$ is the part of $\left(S_{M}^{+}\right)$which is bounded by

$$
\left\{\begin{array}{l}
\xi+m_{0} \log |\lambda|=-K, \\
\xi+m_{1} \log |\lambda|=0 .
\end{array}\right.
$$

$4 .^{\circ} \quad\left(D_{l-1}^{+}\right)$is the part of $\left(S_{M}^{+}\right)$which is bounded by

$$
\left\{\begin{array}{l}
\xi+m_{l-2} \log |\lambda|=0, \\
\xi+m_{l-1} \log \mid \lambda=K
\end{array}\right.
$$

5. In general, $\left(D_{r}^{+}\right)$is the part of $\left(S_{M}^{+}\right)$which is bounded by two co secutive curves of

$$
\left\{\begin{array}{l}
\xi+m_{r} \log |\lambda|=0 \\
\xi+m_{r} \log |\lambda|=0
\end{array} \quad(r=1,2, \ldots, l-3) .\right.
$$

By the relation (4.15) we know that for sufficiently large $M$ all the zero-points of $\lambda^{m} G(\lambda)$ which lie in the half-plane $I(\lambda)>0$ should be located in the region $\left(D_{1}^{+}\right)+\left(D_{2}^{+}\right)+\cdots+\left(D_{n-1}^{+}\right)$, and that

$$
\text { (4.19) }-\sum_{r=0}^{l} n_{r}+\frac{y_{2}-y_{1}}{2 \pi} \sum_{r=0}^{l}\left\{c_{r, n_{r}}-c_{r, 1}\right\} \leqq n\left(y_{1}, y_{2}\right) \leqq \sum_{r=0}^{l} n_{r}+\frac{y_{2}-y_{1}}{2 \pi} \sum_{r=0}^{l}\left\{c_{n, n_{r}}-c_{r, 1}\right\} \text {, }
$$

which is equivalent to

$$
-c+\frac{h_{0}-h_{0}}{2 \pi}\left(y_{2}-y_{1}\right) \leqq n\left(y_{1}, y_{2}\right) \leqq c+\frac{h_{s}-h_{0}}{2 \pi}\left(y_{2}-y_{1}\right)
$$

for we have $c_{0,1}=h_{0}, c_{r, n_{r}}=c_{r+1,1}$, and $c_{l, n_{l}}=h_{s}$, by (4.13).

This cnables us to draw a line which is uniformly bounded far more than $\delta$ from the zero-points of $\lambda^{m} G(\lambda)$ in any strip defined by $I(\lambda)=y$ and $I(\lambda)=y+c$, where $\delta$ depends only on $c$ and is independent upon $y$.

By $L_{r}^{(+)}, L_{r}^{(+,+)}$and $L_{r}^{(+,-)}$, we denote the $r$-th line, and the part of it which belongs to the loft and the right half-planes respectively.

Further let the points of intersection of $L_{r}^{(+)}$with the curves (4.18) be denoted by $a_{r, 0}^{+}, a_{r, 1}^{+} \ldots$ and $a_{r, b-1}^{+}$respectively. We have

$$
R\left(a_{r, 0}^{+}\right)<R\left(a_{r, 1}^{+}\right)<\cdots<R\left(a_{r, l-1}^{+}\right) .
$$

Now in the region $\left(D_{0}^{+}\right)$we draw a circular are defined by

$$
|\lambda|=\left|a_{r, 0}^{+}\right|, \quad \arg \left\{a_{r, 0}^{+}\right\} \leqq \arg \{\lambda\} \leqq \pi,
$$

and in the region $\left(D_{n}^{+}\right)$a circular are defined by

$$
|\lambda|=\left|a_{r, 2-1}^{+}\right|, \quad 0 \leqq \arg \{\lambda\} \leqq \arg \left\{\alpha_{r, l-1}^{+}\right\} .
$$


Quite similarly we may construct the anagolous curve in the lower half-plane $I(\lambda) \leqq 0$, for which the line and the points which correspond to the line $L_{r}^{+}$and the points $a_{r, k}^{+}$may be denoted by $L_{r}^{-}$and $a_{r_{,}}^{-}$. respectively.

Getting two curves in the upper and the lower half-planes we will complete the construction of the contour $\mathfrak{E}_{r}$ by connecting the points $-\left|a_{r, 0}^{+}\right|$and $-\left|a_{r, 0}^{-}\right|$on the negative axis of reals and also by connecting the points $\left|a_{r, l-1}^{+}\right|$and $\left|a_{\vec{r}, l-1}\right|$ on the positive axis of reals by the respective segments on this axis.

Thus constructing a contour $\mathfrak{E}_{r}$, it is casily verified that we are able to get a sequence of such contours $\left\{\mathbb{E}_{r}\right\}$, for which $\mathfrak{E}_{r}$ is contained in the domain enclosed by $\mathfrak{E}_{r+1}$, and that distance between $\mathfrak{E}_{r}$ and $\mathfrak{E}_{r+1}$ are bounded as $r \rightarrow \infty$. (See Fig. 1.).

Here let it be noticed that

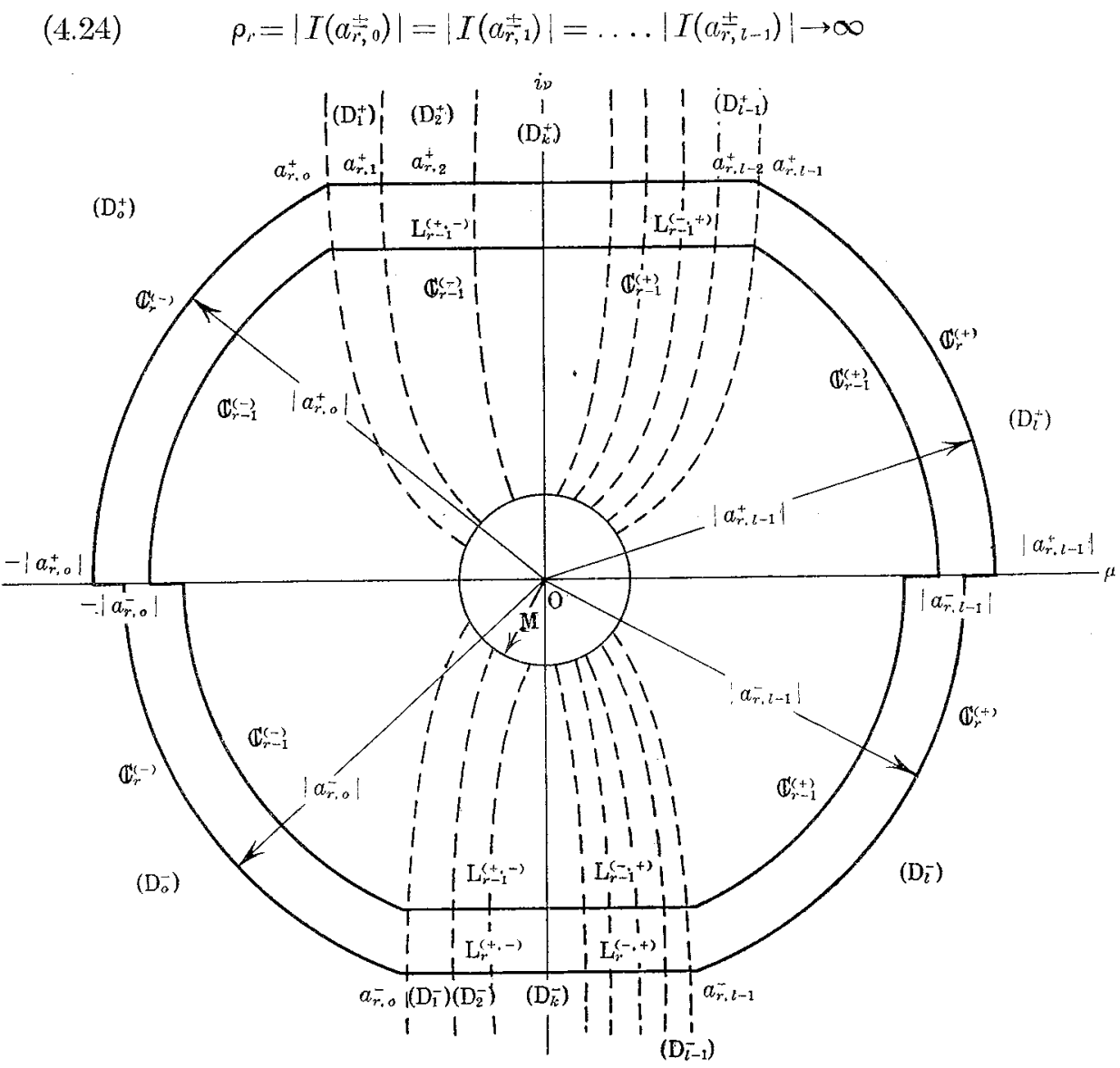

Fig. 1. Construction of Contours $\left\{\mathfrak{e}_{r}\right\}$. 
as $r \rightarrow \infty$.

By the way we remark that if the operator $\Gamma$ is real-valued; that is, $\overrightarrow{G_{k}(\lambda)}=G_{k}(\bar{\lambda})(k=0,1,2, \ldots, n)$ then our contours may be taken symmetric with respect to the real axis.

3. The properties of $\left\{\mathfrak{S}_{r}\right\}$ just now constructed.

We will show that the sequence of contours just constructed iatisfies the following conditions $1^{\circ}, 2^{\circ}$ and $3^{\circ}$, which completes the proof of Theorem IV. I. The conditions $1^{\circ}, 2^{\circ}$ and $3^{\circ}$ read as follows:

$$
\begin{cases}1^{\circ} & d_{r} \uparrow \infty, \\ 2^{\circ} . & \int_{\mathbb{C}_{r}^{( \pm)}}\left|e^{\mp \lambda \varepsilon}\right| d \lambda \mid=O(1), \quad(\text { as } r \rightarrow \infty), \\ 3^{\circ} \cdot & \int_{\mathbb{E}^{\prime \pm} \pm}\left|\frac{e^{\lambda} \mid}{\lambda^{m} G(\lambda)}\right| d \lambda=0(1), \quad(\text { as } r \rightarrow \infty) .\end{cases}
$$

uniformly in the interval $a+\varepsilon \leqq q \leqq b-\varepsilon$. Here $\varepsilon$ in $2^{\circ}$ and $3^{\circ}$ may be chosen as small as we please.

Proof. of (4.25). $1^{\circ}$ is evident, and $2^{\circ}$ followis from the estimations to the effect that

where

$$
\begin{aligned}
& \int_{\mathbb{S}_{,+}^{(+)}}\left|e^{-\lambda \varepsilon}\right||d \lambda| \leqq \int_{a_{r, l-1}^{+}}^{i_{\rho_{r}}}\left|e^{-\lambda \varepsilon}\right||d \lambda|+\underbrace{\int\left|e^{-\lambda \varepsilon}\right||d \lambda|}_{a_{r, l-1}^{+}\left|a_{r, l-1}^{+}\right|} \\
& +\int_{a_{r, l-1}^{-i}}^{-i_{\rho} \rho_{r}}\left|e^{-\lambda \varepsilon}\right||d \lambda|+\underbrace{\int\left|e^{-\lambda \varepsilon}\right|}_{a_{r^{-}, l-1}\left|a_{r^{-}, l-1}^{-}\right|}|d \lambda|+\int_{\left|a_{l^{+}, l-1}^{+}\right|}^{\left|a_{r, l-1}^{-}\right|}\left|e^{-\lambda \varepsilon}\right||d \lambda|
\end{aligned}
$$

(4.262)

$$
\begin{aligned}
& \int_{a_{r, l-1}^{+}}^{i \rho_{r}}\left|e^{-\lambda \varepsilon}\right||d \lambda|=\frac{1}{\varepsilon}\left[1-e^{-\varepsilon R\left(a_{r, l-1}^{+}\right)}\right]=O(1), \\
& \underbrace{\int\left|e^{-\lambda \varepsilon}\right||d \lambda|=O(1),}_{a_{r, l-1}^{+}\left|a_{r, l-1}\right|}
\end{aligned}
$$

and lastly

$$
\int_{\left|a_{r, l-1}^{+}\right|}^{\left|a_{r, l-1}^{-}\right|}\left|e^{-\lambda \varepsilon}\right||d \lambda| \leqq|| a_{r, l-1}^{+}|-| a_{r, l-1}^{-}|| \exp \left\{-\varepsilon \alpha_{r, l-1}\right\}
$$

where we put

$$
\alpha_{r, b-1}=\operatorname{Min}\left(\left|a_{r, b-1}^{+}\right|,\left|a_{r, b-1}^{-}\right|\right)
$$

and we have 
(4.2642)

$$
\begin{gathered}
|| a_{r, l-1}^{+}|-| \alpha_{r, l-1}^{-}||=O(1), \\
\alpha_{r, l-1} \rightarrow \infty
\end{gathered}
$$$$
(\text { as } r \rightarrow \infty \text { ) }
$$

Thus we obtain

$$
\int_{\mathbb{C}_{r}^{(+)}}\left|e^{-\lambda \varepsilon}\right||d \lambda|=O(1), \quad(\text { as } r \rightarrow \infty)
$$

$\boldsymbol{\varepsilon}$ being any given small positive number. The remaining part of $2^{\circ}$ may be quite similarly verified.

Now we have

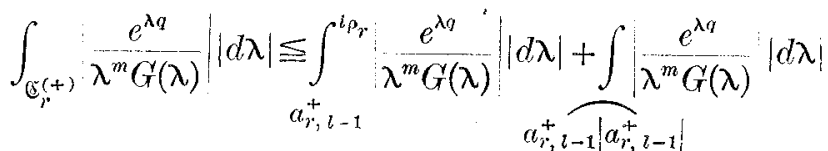

$$
\begin{aligned}
& +\int_{a_{r, l-1}^{-}}^{-i \rho_{r}} \frac{e^{\lambda}}{\lambda^{m} G(\lambda)}|| d \lambda|+\underbrace{\int \lambda^{m} G(\lambda)}_{a_{r, l-1}^{-}\left|a_{r^{\prime}, l-1}\right|}| d \lambda \mid \\
& +\int_{\left|a_{r, l-1}^{-}\right| a_{r, l-1}^{-} \mid}\left|\frac{e^{\lambda q}}{\lambda^{m} G(\lambda) \mid}\right| d \lambda \mid
\end{aligned}
$$

Therefore, we have only to estimate the integrals of the types:

$$
\int_{a_{r, l-3}^{++}}^{i_{\rho_{r}}} \frac{e^{\lambda q}}{\lambda^{m} G(\lambda) \mid}|d \lambda|,
$$

$$
\int_{a_{r, l-1}^{+}\left|a_{r, l-1}^{+}\right|} \frac{e^{\cdot q}}{\lambda^{m} G(\lambda)}|d \lambda|,
$$

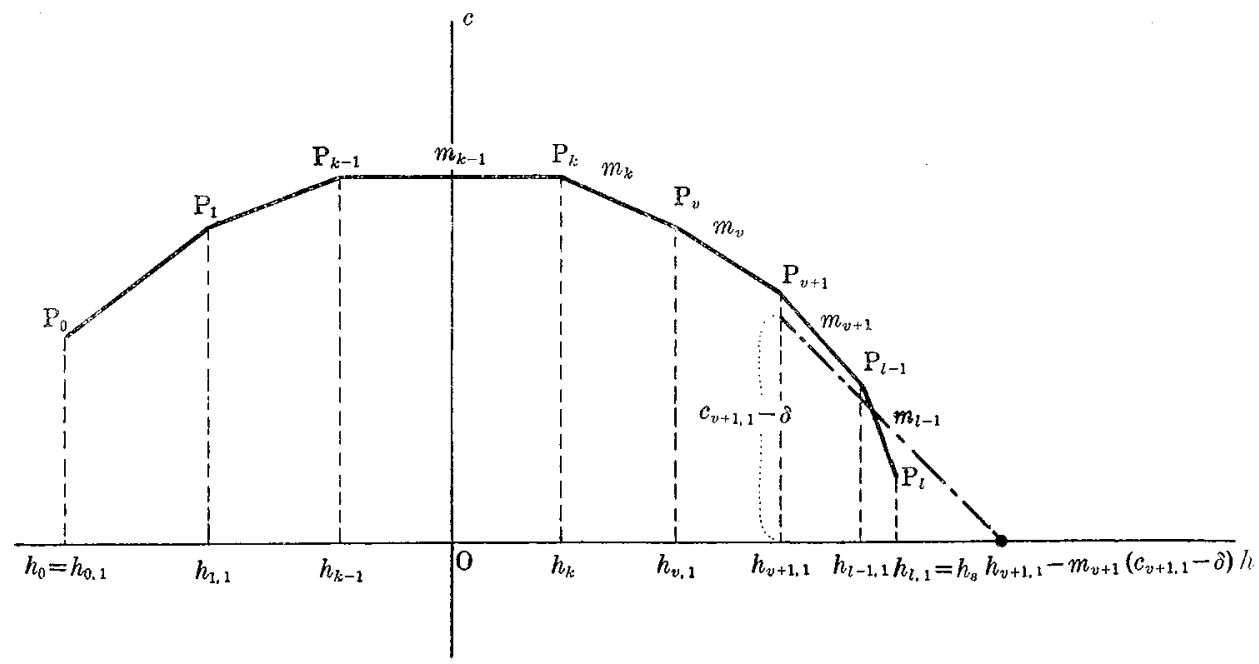

Fig. •. Construction of Broken Line $L$. 
and

$$
\int_{\left|a_{r, l-1}^{+}\right|}^{\left|a_{r, l-2}^{-}\right|}\left|\frac{e^{\lambda q}}{\lambda^{m} G^{\prime}(\lambda)}\right| d \lambda \mid .
$$

Now we will prove that these integrals tend to zero as $r \rightarrow \infty$, uniformly in the interval $a+\varepsilon \leqq q \leqq b-\varepsilon$, where $\varepsilon$ is any given small positive number.

Firstly let us consider the integral (4.28).

Since the region $\left(D_{r}^{(+)}\right)$is contained in the region bounded by two curves (4.09), and therefore (4.10) holds there, we obtain,

$$
\begin{aligned}
& \int_{a_{r, l-1}^{+}}^{L \rho_{r}}\left|\frac{e^{\lambda / q}}{\lambda^{m} G(\lambda)}\right| d \lambda\left|=\int_{R\left(a_{r, l-1}^{+}\right)}^{0} \frac{e^{\mu^{\prime} /}}{\left(\mu+i \rho_{r}\right)^{m} G\left(\mu+i \rho_{r}\right)}\right| d \mu \mid
\end{aligned}
$$

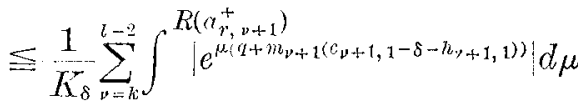

$$
\begin{aligned}
& R\left(a_{r, p}^{+}\right) \\
& +\frac{1}{K_{\delta}} \int_{0}^{R\left(a_{n, k}^{+}\right)} e^{\mu\left(q+m_{k}\left(c_{k, 1}-\delta\right)-h_{k, 1}\right)} d \mu
\end{aligned}
$$

By the convexity of the line $L$ defined by (4.07) and the assumption (4.02) $1^{\circ}$, for any given positive $\varepsilon$, we may choose a positive number $\delta$ such that (Sec Fig. 2.)

$$
-m_{\nu+1}\left(c_{\nu+1,1}-\delta\right)+h_{v+1,1} \geqq h_{l}-\varepsilon, \quad(k \leqq \nu \leqq l-2)
$$

Therefore we obtain

$$
\int_{R\left(a_{r, \nu}^{+}\right)}^{R\left(a_{r, v+1}^{+}\right)}\left|e^{\lambda\left(q+m_{\nu+1}\left(c_{\nu+1}, 1-\delta\right)-n_{v+1}, 1\right)}\right||d \lambda| \leqq \int_{R\left(a_{r, \nu}^{+}\right)}^{R\left(a_{r, \nu+1}^{+}\right)} e^{\mu\left(q+\varepsilon-n_{l}\right)} d \mu,
$$

which is bounded as $r \rightarrow \infty$. Now (4.31) and (4.33) give us

$$
\int_{i \rho_{\gamma,}}^{a_{r, l-1}^{+}}\left|\frac{e^{\lambda q}}{\lambda^{m} G(\lambda)}\right| d \lambda \mid=O\left(\frac{1}{K_{\delta} \rho_{r}^{\delta}}\right),
$$

where $\delta>0$ and $K_{\delta}$ is a positive number independent of $r$. Therefore this tends to zero as $r \rightarrow \infty$.

Next let us consider (4.29). We have 


$$
\overbrace{a_{r, l-1}^{+}\left|a_{r, l-1}^{+}\right|}^{\int \frac{e^{\lambda q}}{\lambda^{m} G(\lambda)}|d \lambda| \leqq-\frac{1}{M_{\delta}} \int\left|e^{\lambda\left(q-h_{\ell}\right)}\right| d \lambda \mid} \underbrace{}_{a_{r, l-1}^{+}\left|a_{r, l-1}^{+}\right|} .
$$

where, putting $a_{r, l-1}^{+}=a_{r} e^{i \theta_{r}}$ and $\lambda=a_{r} e^{i \theta}$, the integral of right-hand side becomes

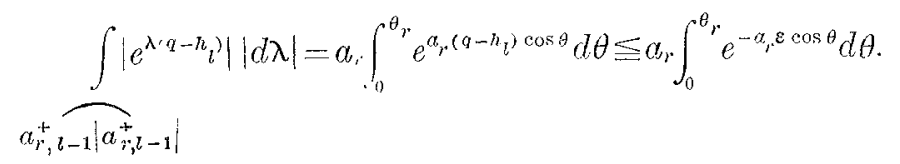

We have

$$
\int_{0}^{\theta} e^{-\sigma^{\varepsilon}} r^{\varepsilon}+\theta d \theta=\int_{\frac{\pi}{2}-\theta_{r}}^{\frac{\pi}{2}} e^{-a_{r} \varepsilon \sin \varphi} d \varphi,
$$

where, as is well known,

$$
\int_{\frac{\pi}{2}-\theta_{r}}^{\frac{\pi}{2}} e^{-a_{r} \varepsilon \sin \varphi} d \varphi \leqq \int_{\frac{\pi}{2}-\theta_{r} .}^{\frac{\pi}{2}} e^{-m \varepsilon \varepsilon \omega_{r}} d \varphi=e^{-m \varepsilon \alpha_{r}, \varphi\left(\frac{\pi}{2}-\theta_{r}\right)} \int_{0}^{\theta_{r}} e^{-m \varepsilon a_{1}, \varphi_{1}} d \varphi_{1},
$$

and, by our construction of the contour,

$$
a_{r}\left(\frac{\pi}{2}-\theta_{r}\right) \rightarrow \infty
$$

Therefore, considering that $m(>0)$ is independent of $\varepsilon$ and $r$, we obtain, by $(4.35)-(4.39)$,

$$
\begin{aligned}
\overbrace{a_{r, l-1}\left|a_{r, l-1}^{+}\right|}^{\int \frac{e^{\lambda q}}{\lambda^{m} G(\lambda)}|| d \lambda \mid} & \leqq a_{r} e^{-m \varepsilon a_{r} \cdot\left(\frac{\pi}{2}-\varphi_{r}\right)} \int_{0}^{\infty} e^{-m \varepsilon a_{r} \varphi_{1}} d \varphi_{1} \\
& =\frac{a_{r}}{m \varepsilon \alpha_{r}} e^{-m \varepsilon a_{r}\left(\frac{\pi}{2}-\varphi_{r}\right)}=\frac{1}{m \varepsilon} e^{-m_{r} \varepsilon a_{r}\left(\frac{\pi}{2}-\varphi_{r}\right)}=o(1) .
\end{aligned}
$$

Lastly let us consider (4.30). We have

$$
\int_{\left|a_{r, l-1}^{++}\right|}^{\left|a_{r, l-1}^{-}\right|}: \frac{e^{\lambda q}}{\lambda^{m} G(\lambda)}|d \lambda| \leqq \frac{1}{M_{\delta}} \int_{\alpha_{r, l-1}}^{\alpha_{r, l-1}+S} e^{-\varepsilon \mu} d u
$$

which, in view of (4.26), tends to zcro as $r \rightarrow \infty$, where

$$
\begin{aligned}
& \alpha_{r, l-1}=\operatorname{Min}\left(\left|a_{r, l-1}^{+}\right|,\left|a_{r, l-1}^{-}\right|\right) \\
& || a_{r, l-1}^{+}|-| a_{r, l-1}^{-1} \mid \leqq S, \quad(r=0,1,2,3, \ldots) .
\end{aligned}
$$

Thus we have proved that the integrals of the types (4.28)-(4.30) tend to zero uniformly in $a+\varepsilon \leqq q \leqq b-\varepsilon$, which, in view of $(4,27)$, yiclds us the proof of $(4.25) 3^{\circ}$. 
The remaining of the theorem may be proved quite similarly.

By the similar arguments we obtain

Theorem IV. II. If, in the hypothesis of Theorem IV.I. (4.02) $x^{\circ}$ is replaced by the condition that

$$
\nu_{i t}=\nu_{s}=\operatorname{Max}_{0 \leqq i \leqq s}\left\{\nu_{i}\right\},
$$

then there is a sequence of contours $\left\{\mathbb{E}_{r}\right\}$ associated to the interval $(+\varepsilon$, $-\varepsilon)$, to which $\lambda^{m} G(\lambda)$ is O-associated with the index $m$ in the interval $(a+\varepsilon, b-\varepsilon)$, where $\varepsilon$ is any given small positive number.

In this case the figure $L$ becomes a segment parallel to the real axis from the point $\left(h_{0}, v_{0}\right)$ to the point $\left(h_{s}, \nu_{s}\right)$, and therefore the proof becomes much simpler. We will further remark that there are positive constants $K$ and $L$ such that

$$
\left|\lambda^{m} G(\lambda)\right| \geqq K\left|e^{\lambda n_{n}}\right|, \quad \text { in } R(\lambda) \geqq R
$$

and

$$
\left|\lambda^{n} G(\lambda)\right| \geqq L\left|e^{\lambda h_{0}}\right|, \quad \text { in } R(\lambda) \leqq-R .
$$

Further in the strip $-R \leqq R(\lambda) \leqq R$, there is a positive number $I_{\delta}$ depending only on $\delta$ such that

$$
\left|\lambda^{m} G(\lambda)\right| \geqq M_{\delta}
$$

provided that $\lambda$ is uniformly bounded (far more than $\delta$ ) from the zero-points of $\lambda^{m} G(\lambda)$. Therefore in this strip we are able to draw a sequonce of lines parallel to the real axis and uniformly bounded more than $\delta$ from the zero-points of $\lambda^{m} G(\lambda)$. In the region $-R \leqq R(\lambda) \leqq R$, we construct the corresponding circular ares as we have done in the domain $\left(D_{0}^{+}\right)+\left(D_{1}^{+}\right)+\cdots+\left(D_{l-1}^{+}\right)$ in the former case and thus in similar manner we may complete the sequence of contours $\left\{\mathfrak{E}_{r}\right\}$.

The different circumstance to be remarkable is only that in this case

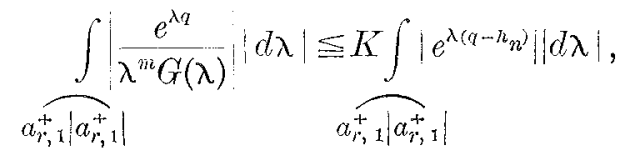

and

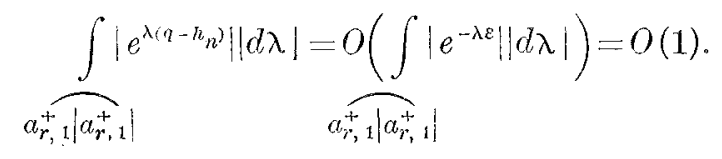

so that we are able to conclude merely $O$-association of the gonerating function to the sequence of contours $\left\{\mathfrak{C}_{r}\right\}$. 


\section{§5. Generating Functions and Their Asymptotic Representations. (Examples to the Convergence-Theorems).}

In this section we will give examples to the convergence-theorems obtained in $\$ 3$.

Example. 1. Let us consider the operator $\Lambda_{1} f(x)$, whose generating function is

$$
G_{1}(\lambda)=\Lambda_{0}\left\{e^{\lambda \xi}\right\}=\int_{a}^{b} e^{\lambda \xi} K(\xi) d \xi
$$

Langer's theorem gives us:

1. If $K(\xi)$ is absolutely continuous, and $K(a)$ and $K(b)$ are not zero, then we have

$$
G_{1}(\lambda)=\frac{1}{\lambda}(K(b)+o(1)) e^{\lambda i}-\frac{1}{\lambda}(K(a)+o(1)) e^{\lambda a} .
$$

2. If $K(\xi)$ is n-time differentiable, $K^{(n)}(\xi)$ is absolutely continuous, and $K^{(s)}(a)=K^{(s)}(b)=0,(s=0,1,2, \ldots, n-1), K^{(n)}(a) \neq 0$, and $K^{(n)}(b) \neq 0$, then we have

$$
G_{1}(\lambda)=\frac{1}{\lambda^{n}}\left(K^{(n)}(b)+o(1)\right) e^{\lambda^{\prime}}-\frac{1}{\lambda^{n}}\left(K^{(n)}(a)+o(1)\right) e^{\lambda a} .
$$

Thus $\lambda^{n} G_{1}(\lambda)$ has the asymptotic representation assumed in the previous section, and we are able to get the convergence-theorems with respect to this operator $\Lambda_{1}$ under these special conditions.

3. J. Delsarte [1]-[5] called a function $f(x)$ defined in $(-\infty, \infty)$ to be mean-periodic (moyenne-périodique) if it satisfies the relation

$$
\Lambda f(x) \equiv \int_{0}^{a} f(x+t) K(t) d t=0, \quad(-\infty<x<\infty) .
$$

He obtained the expansion-theorem with the linear translatable operator (in our terminology) to the following effect $\left({ }^{38}\right)$ : let us assume that $K(t)$ satisfy the following three conditions:

$$
\begin{cases}\text { 1. } & K(t) \text { is of bounded variation. } \\ \text { 2. } & K(0+0), K(a-0) \neq 0 \\ \text { 3. } & \text { The continuity-part } \Re(x) \text { of } K(x) \text { is absolutely continuous. }\end{cases}
$$

The "présque-dérive" ' $\mathfrak{K}(x)$ of $K(x)$ is integrable in the sense of Riemann's. The set of the points where 'si(x) becomes infinity is at most of measure zero.

Let $f(x)$ be of bounded variations in $(0, a)$ and let

(:8) Delsarte [5] p. 444-445. 


$$
\int_{19}^{a} f(t) K(t) d t=0
$$

Then the Cauchy's series (in our terminology) of $f(x)$ with respect to the operator $\Lambda_{1} f(x)$ is convergent in the interval $0<x<a$, and has the limiting value $\{f(x-0)+f(x+0)\} / 2$.

Furthermore this series converges uniformly in any interval contained in the interior of the interval $(0, a)$, if $f(x)$ is continuous in this partial interval.

Here we will remark that this theorem may be recognised as a special case of Theorem III. III.

Indeed, putting $n=m=0$, and noticing that the conditions (3.16) and (3.17) are satisfied by the assumption, we have only to verify that the assumptions with respect to the generating function in Theorem III. III. are satisfied, in order to deduce the theorem of Delsarte's from Theorem III. III. t that is to say, we have only to assertain that there is a sequence of contours $\left\{\mathfrak{S}_{r}\right\}$ such that

$$
\left\{\mathfrak{S}_{r}\right\} \& \mathfrak{C}\left(\delta_{1},-\delta_{2}\right)
$$

and, with respect to this sequence

$$
G(\lambda)=\mathfrak{C}_{r}-O\left(1, \delta_{1}^{\prime}, a-\delta_{2}^{\prime}\right)
$$

where $\delta_{1}, \delta_{2}, \delta_{1}^{\prime}$ and $\delta_{2}^{\prime}$ are sufficiently small positive numbers such that the interval ( $\operatorname{Max}\left(\delta_{1}, \delta_{1}^{\prime}\right), a-\operatorname{Max}\left(\delta_{2}, \delta_{2}^{\prime}\right)$ ) contains the given interval contained in the interior of the interval $(0, a)$.

Example 2. Let us consider the operator $\Lambda_{f} f(x)$, whose generating function is

$$
G_{2}(\lambda)=\int_{a-0}^{b+0} e^{\lambda t} d \varphi(t), \quad(a<b) .
$$

Recently R. C. Young [1]( $\left.{ }^{39}\right)$ studied the asymptotic behaviour of this function. By her transformation-formula it is convenient to express (5.04) in the form

$$
G_{::}(\lambda)=\left(\lambda_{0}-\lambda\right) \int_{a}^{b} e^{\lambda t} H(t) d t
$$

where $\lambda_{0}$ is a zoro-point of $G_{1}(\lambda)$ and

$$
H(t) \equiv H\left(t, \lambda_{\theta}\right)=e^{-\lambda_{0} t} \int_{a-0}^{t+9} e^{\lambda_{0} \tau} d \varphi(\tau) .
$$

Thus the consideration of Example 1 may be applicable, which we

(39) R. C. Young [1], § The asymptotic behaviour of $F(z)\left(=\int_{a-0}^{b+0} e^{\lambda t} d \varphi(t)\right)$. 
will not repeat here.

On the other hand it will be more interesting to give certain special cases as follows:

Case $I$. Let $\varphi(t)$ be a step-function with finite number of discontinuitypoints. Then

$$
G_{2}(\lambda)=\sum_{i=0}^{n} A_{i} e^{\lambda t_{i}}
$$

Specially when $n=1, h_{n}=-\pi$, and $h_{1}=\pi$, it reduces to the case of Fourier's series.

Case IT. Let $\varphi(t)$ be expressed in the form

$$
\varphi(t)=s(t)+g(t)
$$

where $s(t)$ is a step-function (everywhere continuous on the left) with finite number of discontinuity-points and $g(t)$ is absolutely continuous in the interval ( $a b)$; and $g^{\prime}(\alpha) \neq 0, g^{\prime}(b) \neq 0$.

This is the case which we have discussed in our previous paper (Kitagawa [1]) by another method. As we have proved there, the generating function has the following asymptotic representation:

$$
G(\lambda)=\sum_{=0}^{n} A_{k} e^{\lambda t_{k}}+\frac{1}{\lambda}\left(g^{\prime}(b)+o(1)\right) e^{\lambda b}-\frac{1}{\lambda}\left(g^{\prime}(a)+o(1)\right) e^{\lambda a} .
$$

Example 3. Let us consider the linear translatable differential operator $\Gamma f(x)$ defined by

$$
\Gamma f(x)=\sum_{k=0}^{n} \int_{a-0}^{b+n} f^{(k)}(x+t) d \varphi_{k}(t)
$$

whose generating function becomes

$$
G(\lambda)=\sum_{k=0}^{n} \lambda^{k} \int_{a-0}^{b+0} e^{\lambda t} d \varphi_{k}(t)
$$

When these functions $\varphi_{k}(t)$ satisfy some of the conditions in Example 2 , then $G(\lambda)$ may be expressed in an asymptotic exponential sum, to which the consideration in $\$ 4$ may be applicable.

Let it be noticed that, when all $\varphi_{k}(t)$ reduce to step-functions with finite number of discontinuity-points, then we have

and

$$
\Gamma f(x)=\sum_{\mu=0}^{n} \sum_{\nu=0}^{m} a_{\mu, v} f^{(\mu)}\left(x+t_{v}\right)
$$

$$
G(\lambda)=\sum_{\mu=0}^{n} \sum_{n=0}^{m} a_{\mu, \nu} \lambda^{\mu} e^{\lambda t},
$$

which is the casc treated by Hilb [1].

Thus Hilb's convergence-theorems are completely included in the discussion developed hitherto. 


\section{Chapter II. Solutions of the Linear Translatable Functional Equation under Various Conditions.}

\section{§6. Convergence-theorems and Solutions of Linear Translatable Functional Equation.}

Let us consider the functional equation

$$
\Gamma_{\xi}\{f(x+\xi)\}=0, \quad\left(x_{0}+\alpha<x<x_{0}+\beta\right),\left({ }^{41}\right)
$$

which contains, as its special case, the difference-differential equation with constant coefficients:

$$
\sum_{\mu=0}^{n} \sum_{\nu=11}^{m} \omega_{\mu, v} f^{(\mu)}\left(x+h_{\nu}\right)=0\left(^{12}\right), \quad\left(x_{0}+\alpha<x<x_{0}+\beta\right) .
$$

As readily seen, any section of Cauchy's series with respect to the linear translatable operator $\Gamma$ satisfies the equation (6.01). We wish to expand solutions of (6.01) in this series of Cauchy's, using the results which we have established in $\$ 3$.

To apply the convergence-theorem: in $\$ 3$ to our problem, we remember that, as to the properties of $f(x)$ to be expanded, these theorems concern themselves with the following two questions:

1. To what function-set $f(x)$ belongs?

2. Whether $f(x)$ satisfies the boundary condition

$$
B_{s}\left(f ; x_{0}\right)=0
$$

or not?

Now let it be noticed that to satisfy the so-called boundary condition (6.03) at the point $x_{0}$ is nothing but to satisfy the functional equations

$$
\Gamma_{\xi}\left\{f^{(k)}(x+\xi)\right\}=0 \quad(k=0,1,2, \ldots, s-1)
$$

at the point $x_{0}$.

Further we remark that, if $f(x)$ is $n+m$-time differentiable in $\left(x_{0}+\alpha+a\right.$, $\left.x_{0}+\beta+b\right)$, and satisfies the functional equation (6.01), then $f(x)$ satisfies (6.04) in $\left(x_{0}+\alpha, x_{0}+\beta\right), s$ being equal to $n+m$, which follows from the fact that, for the linear translatable operator in our consideration, we are permitted to change the order of differentiation and the operator $\Gamma$, that is,

$$
\Gamma f^{s \prime}(x)=\frac{d^{s}}{d x^{s}}\{\Gamma f(x)\}
$$

provided that $f^{(s)}(x)$ exists and belongs to the domain of $\Gamma$ in the interval

(41) Without loss of generality, we may -and we shall assume $\alpha<0<\beta$.

(42) Without loss of generality, we may assume $a=h_{0}<h_{1}<h_{2}<\cdots<h_{m}=b$. 
$\left(x_{0}+a+\alpha, x_{0}+b+\beta\right)$.

Thus we may deduce a group of expansion theorems of the solutions of the functional equation (6.01) into its Cauchy's series from the convergence-theorem in $\$ 3$, which, however, wo will not repeat here. We wish rather to give two theorems which are fundamental in the sequel and which read as follows:

Theorem VI. I. Let $f(x)$ be a solution of the functional equation (6.01) in the interval $\left(x_{0}+a+\alpha, x_{0}+b+\beta\right)$. Let it be possible to interchange the order of definite integration of $f(x)$ and the operators $\Lambda^{k}$ by Property III.

Then the coefficients of the expansion of $f(x)$ in its Cauchy's series with respect to the initial point $\zeta$ are independent of $\zeta$, provided that $\zeta$ lies in the open intervtio $\left(x_{0}+\alpha, x_{0}+\beta\right) .\left({ }^{43}\right)$

To prove this, we write

(6.06) $K(\lambda, \zeta ; f) \equiv \sum_{k=1}^{n} \Lambda_{0}^{k}\left\{e^{\lambda \xi} \int_{\zeta}^{\zeta+\xi} e^{-\lambda \eta} f^{(k)}(\eta) d \eta\right\}+\sum_{k=1}^{n} \lambda^{k} G_{h}(\lambda) \sum_{\nu=0}^{k-1} \frac{f^{(\nu)}(\zeta)}{\lambda^{p+1}}$,

and we consider the difference

$$
K\left(\lambda, \zeta_{2} ; f\right)-K\left(\lambda, \zeta_{1} ; f\right)
$$

where $\zeta_{1}$ and $\zeta_{2}$ are any two numbers such that $x_{0}+\alpha<\zeta_{1}<\zeta_{2}<x_{j}+\beta$.

Changing the order of the operator $\Lambda^{k}$ and the integration of $f(x)$, we have

$$
\begin{aligned}
\Lambda_{\xi}^{k}\left\{e^{\lambda \xi} \int_{\zeta_{2}}^{\zeta_{2}+\xi} e^{-\lambda \eta} f^{(k)}(\eta) d \eta\right\} \\
=\Lambda_{\xi}^{k}\left\{e^{\lambda \xi}\left(\int_{\zeta_{2}}^{\zeta_{1}}+\int_{\zeta_{1}}^{\zeta_{1}+\xi}+\int_{\zeta_{1}+\xi}^{\zeta_{2}+\xi}\right) e^{-\lambda \eta} f^{(k)}(\eta) d \eta\right\} \\
=G_{\zeta_{1}}(\lambda) \int_{\zeta_{2}}^{\zeta_{1}} e^{-\lambda \eta} f^{(k)}(\eta) d \eta+\Lambda_{\xi}^{k}\left\{e^{\lambda \xi} \int_{\zeta_{1}}^{\zeta_{1}+\xi} e^{-\lambda \eta} f^{(k,}(\eta) d \eta\right\} \\
\\
\quad+\int_{\zeta_{1}}^{\zeta_{2}} e^{-\lambda \zeta} \Lambda_{0}^{k \xi}\left\{f^{(h)}(\xi+\zeta)\right\} d \zeta .
\end{aligned}
$$

Combining (6.07) and 6.08), we get, in view of (1.04),

$$
\begin{aligned}
K\left(\lambda, \zeta_{2} ; f\right)-K\left(\lambda, \zeta_{1} ; f\right) \\
=\sum_{k=0}^{n} G_{k}(\lambda) \int_{\zeta_{2}}^{\zeta_{1}} e^{-\lambda \eta} f(\eta) d \eta+\sum_{k=1}^{\prime \prime} \int_{\zeta_{1}}^{\zeta_{2}} e^{-\lambda \zeta} \Lambda_{\xi}^{k}\left\{f^{k)}(\xi+\zeta)\right\} d \zeta \\
\quad+\sum_{k=1}^{n} \lambda^{k} G(\lambda) \sum_{\nu=0}^{k-1} \frac{f^{\nu)}\left(\zeta_{2}\right)}{\lambda^{\nu+1}}-\sum_{k=1}^{n} \lambda^{k} G_{k}(\lambda) \sum_{\nu=0}^{k-1} \frac{f^{(\nu)}\left(\zeta_{1}\right)}{\lambda^{\nu+1}} \\
=G(\lambda) \int_{\zeta_{2}}^{\zeta_{1}} e^{-\lambda \eta} f(\eta) d \eta+\sum_{k=1}^{n} \int_{\zeta_{1}}^{\zeta_{2}} e^{-\lambda \zeta} \Lambda_{\xi}^{k}\left\{f^{(k)}(\xi+\zeta)\right\} d \zeta,
\end{aligned}
$$

$\left({ }^{+3}\right)$ I owe this proof to Prof. Nagumo's suggestion.

By imposing the suitable conditions upon either or both of $\Lambda$ and $f(x)$, we are able to prove the possibility of this interchange of the order of $A$ and the definite integration of $f(x)$. 
where, since $x_{0}+\alpha<\zeta_{1}<\zeta_{2}<\alpha_{0}+\beta$, we have

$$
\left.\sum_{k=0}^{n} \int_{\zeta_{1}}^{\zeta_{2}} e^{-\lambda \eta} \Lambda_{0}^{k}\left\{f^{(k)}(\xi+\zeta)\right\} d \zeta=\int_{\zeta_{1}}^{\zeta_{2}} e^{-\lambda \zeta} \Gamma f ! \zeta\right) d \zeta=0
$$

Therefore

$$
\begin{aligned}
\mathrm{T}_{r}\left(x, \zeta_{2} ; f\right)-\mathrm{T}_{r}\left(x, \zeta_{1} ; f\right) \\
\quad=\frac{1}{2 \pi i} \oint_{\mathcal{C}_{r}} \frac{e^{\lambda x}}{G(\lambda)}\left\{K\left(\lambda, \zeta_{2} ; f\right)-K\left(\lambda, \zeta_{1} ; f\right)\right\} d \lambda \\
\quad=\frac{1}{2 \pi i} \oint_{\mho_{r}} e^{\lambda x}\left(\int_{\zeta_{2}}^{\zeta_{1}} e^{-\lambda \zeta} f(\zeta) d \zeta\right) d \lambda=0,
\end{aligned}
$$

which shows that the coefficients of the Cauchy's series of $f(x)$ with respect to the initial point $\zeta_{2}$, are equal to those corresponding coefficients of the Cauchy's series of $f(x)$ with respect to the initial point $\zeta_{1}$.

Since $\zeta_{1}$ and $\zeta_{2}$ are any two numbers in the open interval $\left(x_{0}+\alpha, x_{0}+\beta\right)$, we have thus proved our theorem completely.

Theorem VI. II. Let $f(x)$ be a solution of the functional equation

$$
\Gamma_{\xi}\{f(x+\xi)\}=0, \quad\left(x_{0}+\alpha<x<x_{0}+\beta\right),
$$

and let it be continuous and of bounded variation in the interval $\left(x_{0}+\alpha+\alpha\right.$, $\left.x_{0}+b+\beta\right)$. Let it be possible to interchange the order of definite integration of $f(x)$ and the operator's $\Lambda_{k}(k=0,1, \ldots, n)$ by Property III.

Further let us assume that there is a sequence of contours such that

$$
\left\{\mathbb{S}_{1}\right\} \in \mathbb{S}\left(\delta_{i},-\delta_{2}\right)
$$

and that, with res ect to this sequence $\left\{\mathbb{E}_{r}\right\}$

$$
G(\lambda)=\aleph_{r}-O\left(1 ; a+\delta_{1}{ }^{\prime}, b-\delta_{2}{ }^{\prime}\right) .
$$

Then $\left\{\mathrm{T}_{i}\left(x, x_{0} ; f\right)\right\}$ defined for this sequence $\left\{\mathcal{S}_{,}\right\}$tends to $f(x)$ uniformly concerning $x$ in the interval $\left(x_{1}+\alpha+\delta_{1}{ }^{\prime \prime}+\varepsilon, x_{11}+\beta-\delta_{2}{ }^{\prime}-\varepsilon\right), \varepsilon$ being any given positive number, and $\delta_{1}^{\prime \prime}=\operatorname{Max}\left(\delta_{1}, \delta_{1}{ }^{\prime}\right) . \delta_{2}{ }^{\prime \prime}=\operatorname{Max}\left(\delta_{2}, \delta_{2}^{\prime}\right)$.

The convergence-theorem in $\S 3$, Theorem III.I., yields us that $\mathrm{T}_{r}(x, \zeta ; f)$ defined for the sequence $\left\{\widetilde{\mho}_{r}\right\}$ with the initial point $\zeta$ tends to $f(x)$ uniformly in the closed interval $\left[\zeta+a+\operatorname{Max}\left(\delta, \delta_{1}{ }^{\prime}+\varepsilon, \zeta+b-\operatorname{Max}\left(\delta_{-}, \delta_{2}{ }^{\prime}\right)-\varepsilon\right]\right.$, where $\varepsilon$ may bo any small positive number, but we assume that $\varepsilon$ is suitably chosed and fixed. Thus we are able to correspond to any point $\zeta$ in the interval $\left[x_{0}+\alpha+\varepsilon, x_{0}+\beta-\varepsilon\right]$ the interval $\left[\zeta+a+\operatorname{Max}\left(\delta_{1}, \delta_{1}{ }^{\prime}\right)+\varepsilon, \zeta+b-\operatorname{Max}\right.$ $\left.\left(\delta_{:}, \delta_{:}^{\prime}\right)-\varepsilon\right]$, which contains $\zeta$ as the interior point and in which $\mathrm{T}_{r}(x, \zeta ; f)$ tends uniformly to $f(x)$ as $r \rightarrow \infty$, for $f(x)$ is continuous and of bounded variation in $\left(x_{0}+a+\alpha+\varepsilon, x_{0}+b+\beta-\varepsilon\right)$.

By Heine-Borel's covering theorem the interval $\left[x_{0}+\alpha+\delta_{1}^{\prime \prime}+\varepsilon, x_{0}+\beta-\right.$ 
$\left.\delta_{2}^{\prime \prime}-\varepsilon\right]$ may be covered by finite number of such intervals.

Now, since we have, by Theorem VI.I.,

$$
\mathrm{T}_{r}(x, \zeta ; f)=\mathrm{T}_{r}\left(x, x_{\theta} ; f\right),
$$

as a consequence of the assumption that $f(x)$ is a solution of (6.12), we see that $\mathrm{T}_{r}\left(x, x_{1} ; f\right)$ tends to $f(x)$ as $r \rightarrow \infty$ uniformly in the interral $\left(x_{0}+\alpha+\delta_{1}{ }^{\prime \prime}+\varepsilon\right.$, $\left.x_{0}+\beta+\delta_{2}^{\prime \prime}-\varepsilon\right)$, which was to be proved.

\section{$\S 7$. Infinitely Many Time Differentiable Solutions and Analytic Solutions.}

Let $f(x)$ be a solution of the functional equation

$$
\Gamma_{\xi}\{f(x+\xi)\}=0
$$

in the interval $x_{n}+\alpha<x<x_{1}+\beta$. Further let us assume that $f(x)$ is indefinitely many time differentiable in $\left(x_{0}+a+\alpha, x_{0}+b+\beta\right)\left({ }^{4+4}\right)$.

Under these assumptions it follows that

$$
\Gamma_{0}\left\{f^{(s)}(x+\xi)\right\}=0 \quad(s=0,1,2, \ldots)
$$

in the interval $\left(x_{0}+\alpha, x_{0}+\beta\right)$, and $f^{\prime}(x)$ belongs to the function.set $\left(\mathscr{L}_{m}\right)$ $\left(x_{0}+a+\alpha, x_{3}+b+\beta\right)$, however large $m$ may be. Therefore a slight modification of the convergence theorem in $\$ 3$ yields us, in view of (1.19), the following theorems:

Theorem VII. I. In addition to the above assumptions of this paragraph, let us assume that there are a sequence of contours $\left\{\mathbb{S}_{r}\right\}$ associated to the interval $(a+\varepsilon,-b+\varepsilon)$ and a sequence of positive integers $\{m(r)\}$ such that

$$
\sum_{p=1}^{m(r)+n^{\prime-1}} \frac{\left|f^{(\nu)}(x)\right|}{d_{r}{ }^{p}}=O(1), \quad(\text { as } r \rightarrow \infty),
$$

and

$$
\int_{\mathbb{C}_{r}^{( \pm)}} \frac{e^{\lambda q}}{\lambda^{m(r)} G(\lambda)} \cdot d \lambda \mid=O(1), \quad(\text { as } r \rightarrow \infty),
$$

uniformly in the interval $-\varepsilon<q<\varepsilon, \varepsilon$ being a positive number.

Then the contour-integral $\mathrm{T}_{r}\left(x, x_{\|} ; f\right)$ tends to $f(x)$ uniformly in the interral $\left(x_{0}+\alpha+2 \varepsilon, x_{0}+\beta-2 \varepsilon\right)$ for any given positive number $\varepsilon$ as $r \rightarrow \infty$.

Theorem VII. II. Let $f(x)$ be analytic in the interval $\left(x_{0}+\alpha+a\right.$, $\left.x_{0}+\beta+b\right)$, and let it be a solution of the functional equation (6.01) in $\left(x_{0}+\alpha, x_{1}+\beta\right)$.

Further let us assume that there is a sequence of contours $\left\{\mathfrak{C}_{\text {, }}\right\}$ associated to the interval $(a+\varepsilon,-b+\varepsilon)$ such that

(4t) For an infinitely many time differentiable function $f(x)$, we can easily prove the possibility of the interchange of the order of in'egration and linear translatable operator. 


$$
\int_{\mathbb{E}^{\prime}, t} \frac{e^{\lambda q}}{\lambda^{\eta^{\prime} r^{d}} \cdot G(\lambda)}|d \lambda|=O(1), \quad(\text { as } r \rightarrow \infty),
$$

where $q$ is any number in $(-\varepsilon, \varepsilon)$ and $\left\{\eta_{r}\right\}$ is a sequence of positive numbers such that

$$
\left.\eta_{r} \geqq \eta>0, \quad \text { (for } r \geqq R\right) .
$$

Then the contour-integral $\mathrm{T}_{r}\left(x, x_{0} ; f\right)$ tends to $f(x)$ uniformly in the interral $\left(x_{11}+\alpha+2 \varepsilon, x_{0}+\beta-2 \varepsilon\right)$ as $r \rightarrow \infty$.

To prove this, we have only to verify that the conditions (7.03) and (7.04) are satisfied.

For this purpose we will remind that, for a regular function $f(x)$, there are constants $M$ and $p$ independent of $\nu$ such that

$$
\left|f^{(\nu)}(x)\right|<\frac{M \nu !}{p^{v}}
$$

in $\left(x_{1}+a+\alpha, x_{1}+b+\beta\right)$.

Thus we have

$$
\sum_{\nu=0}^{m(r)+n^{-1}} \frac{\left|f^{\nu}(x)\right|}{d_{r}^{\nu}}<M \sum_{\nu=11}^{m(r)+n-1} \frac{\nu !}{\left(p d_{r}\right)^{\nu}}
$$

where the sum of the right-hand side may be estimated as follows:

$$
\sum_{\nu=0}^{m(r)+n-1} \frac{\nu !}{\left(p d_{r}\right)^{r}} \leqq \sum_{\nu=0}^{m(r)+n-1}\left(\frac{\nu e^{-1}\left(1+\frac{S}{\nu+1}\right)}{p d_{r}}\right)^{v},
$$

where $S$ is a constant independent on $\nu$.

Combining (7.08) and (7.09) we get

$$
\sum_{v=0}^{m(r)+n-1} \frac{\left|f^{(\nu)}(\dot{x})\right|}{d_{r}{ }^{v}}<M \sum_{\nu=11}^{m(r+n-1}\left(\frac{\nu P}{d_{r}}\right)^{v}
$$

where we put

$$
P=\frac{(S+1) e^{-1}}{p}
$$

Now let us choose the scquence of integers $\{m(r)\}$ such that

$$
0<\frac{\gamma}{2} \leqq \frac{P(m(r)+n-1)}{d_{r}} \leqq \gamma<1,
$$

where $\gamma$ is a constant independent of $r$.

Then we get

$$
\sum_{\nu=0}^{n(r)+n-1}\left(\frac{P \nu}{d_{r}}\right)^{\nu} \leqq \sum_{\nu=0}^{\infty} \gamma^{v}=\frac{1}{1-\gamma}<\infty
$$

and therefore 


$$
\sum_{\nu=0}^{m, r)+n-1} \frac{\left|f^{\nu}(x)\right|}{d_{r}{ }^{\nu}}=O(1)
$$

as $r \rightarrow \infty$, where $x_{0}+a+\alpha<x<x_{i 1}+b+\beta$.

Also by (7.12) we have

$$
m(r) \geqq \frac{\gamma}{2 P} d_{r}-(n-1)>\frac{\gamma}{4 P} d_{r}
$$

where $r>R$.

By the assumption (7.05) there is a sequence of contours associated to the interval $(a-\varepsilon,-b+\varepsilon)$ and satisfying (7.05).

Thus putting $\eta=\frac{\gamma}{4 P}$, we obtain

$$
\begin{aligned}
\int_{\tilde{C}_{r}^{(+)}} \frac{e^{\lambda q}}{\lambda^{\left.m()^{\prime}\right)} G(\lambda)}|d \lambda| & =O\left(\int_{\tilde{C}_{r}^{\prime} \pm} \frac{e^{\lambda q}}{\lambda^{\prime \prime} r G(\lambda)}|d \lambda|\right) \\
& =O(1),
\end{aligned}
$$

as $r \rightarrow \infty$.

By (7.14) and (7.16) we see that the conditions of Theorem VII.I. are satisfied, which proves the theorem.

To apply this theorem, it may be worth while to mention

Theorem VII. III. If the generating function $G(\lambda)$ is an integral function of the order $\rho$ less than 1, then, in the hypothesis to Theorem VI.IT., the assumptions concerning the behaviour of $G(\lambda)$ are satisfied, and therefore the conclusion holds.

To prove this, we remind a well-known theorem of integral functions to the following effect: let $g(\lambda)$ be an integral function of the order $\rho$. If we draw circles about its zero-points $a_{i}$ with radii $\left|a_{k}\right|^{-h}(h>\rho)$ respectively, then, outside of these circles, we have, for any given positive $\varepsilon$,

$$
|g(\lambda)|>e^{-\left.\lambda\right|^{\rho+\varepsilon}}
$$

provided that $|\lambda| \geqq R, R$ being sufficiently large number.

In virtue of this theorem, we are able to draw a sequence of circles $\left\{\mathfrak{E}_{r}\right\}$ with radii $d_{r}$ such that, for sufficiently large $r$,

$$
\mid \frac{1}{G, \lambda)}<e^{\mid v^{p+\varepsilon}}, \quad\left(j \lambda \mid=d_{r}\right)
$$

and

$$
0<\rho+\varepsilon<1
$$

Therefore

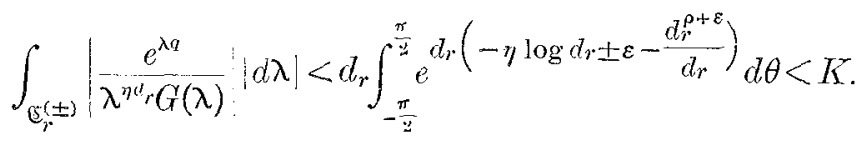


Further it may be easily verified that this sequence of contours satisfies condition $1^{\circ}$ and $2^{\circ}$, and therefore we get our theorem.

Here let it be noticed that from this theorem we may deduce another proof of the expansion-theorems in the Ritt's theory of the linear differential equation of the infinite order. $\left({ }^{45}\right)$ (See $\left.\$ 9 . p .280\right)$

\section{$\S 8$. The Order of Magnitude of Solutions and Schürer's Theorem.}

We will begin with

Theorem VIII. I. Let $f(x)$ be a solution of the functional equation

$$
\Gamma_{0}\{f(x+\xi)\}=0
$$

for every $x$ in $(0, \infty)$ and let $f^{(n)}(x)$ be integrable in any finite interval on $(0, \infty)$. Let it be possible to interchange the order of a definite integration of $f(x)$ and the operator $\Gamma$ by Property III. Let, for each $x, E_{x}$ possess the norm (0.17), and let there be constants $M_{k}$ independent of $x$ and function $f(t)$ such that

$$
\left|\Lambda^{k} f^{(k}(x)\right| \leq M_{k}|| f^{(k)}(t):\left.\right|_{x} \quad(k=0,1,2, \ldots, n)
$$

If furthermore

$$
\lim _{x \rightarrow \infty} \frac{\log \left|f^{(\nu)}(x)\right|}{x}=\alpha_{\nu} \leqq n, \quad(\nu=0,1,2, \ldots, n),
$$

then the coefficients of Cauchy's series of $f(x)$ with respect to the linear translatable operator $\Gamma$ which correspond to the characteristic roots $\lambda_{n}$ of the generating function, such that

$$
P\left(\lambda_{n}\right)>s
$$

are equal to zero.

Proof. We will prove this theorem by showing that, in the residues given by (6.06), those which correspond to the characteristic roots such as (8.04) is satisfied, are equal to zero. Let $\lambda_{m}$ be a simple root of the characteristic equation. Then the corresponding coefficient $a_{m}$ will be given

$$
\begin{aligned}
a_{m}= & \frac{1}{G^{\prime}\left(\lambda_{m}\right)} \sum_{k=0}^{n} \Lambda_{\xi}^{k}\left\{e^{\lambda} m^{\xi} \int_{x_{0}}^{x_{0}+\xi} e^{-\lambda_{m} \eta} f^{(k)}(\eta) d \eta\right\} \\
& +\frac{1}{G^{\prime}\left(\lambda_{m}\right)} \sum_{k=1}^{n} \lambda_{m}^{k}\left(X^{\prime}\left(\lambda_{m}\right) \sum_{\nu=0}^{k-1} \frac{e^{-\lambda_{m} x_{0}} f^{\nu)}\left(x_{0}\right)}{\lambda_{m}^{\nu+1}}\right.
\end{aligned}
$$

which is independent of $x_{i}$, as we have shown in Theorem VI.I.

Consequently we may select $x_{0}$ as large as we please.

( $\left.{ }^{5}\right)$ Ritt [1]. Specially see \$5. Multip.ication and factorization of operators. (p. 36). $\S 6$. Comparison with the equation of finite order. $(37-39)$. $\$ 7$. Determination and identification of the formal development p. 39-41. See also Appendix $I$. 
In virtue of the assumption (8.02), for any given positive number $\varepsilon$, there is a positive number $K(\varepsilon)$ such that we have, for $\eta>K(\varepsilon)+\alpha$,

$$
\left|e^{-\lambda_{m} \eta} f^{(k)}(\eta)\right|<\varepsilon . \quad(k=0,1,2, \ldots, n)
$$

Therefore we know that $\left|a_{m}\right|$ is not greater than

$$
\frac{\varepsilon}{\left|G^{\prime}\left(\lambda_{m}\right)\right|} \sum_{k=0}^{n} M_{k}\left\|e^{\lambda_{m} \xi}|\xi|\right\|_{0}+\frac{\varepsilon}{\left|G^{\prime}\left(\lambda_{m}\right)\right|} \sum_{k=1}^{n}\left|\lambda_{m b}^{k} G_{k}\left(\lambda_{m}\right)\right| \sum_{\nu=0}^{k-1} \frac{1}{\left|\lambda_{m}\right|^{p+1}},
$$

where $\varepsilon$ may be as small as we please. This leads us to

$$
a_{m}=0 \text {, }
$$

which was to be proved. When $\lambda_{m}$ is a multiple root of the characteristic equation, we may proceed quite similarly. Thus our theorem is completely proved. Further we may prove similarly

Theorem VIII. II. Let $f(x)$ be a solution of the functional equation

$$
\Gamma_{\xi}\{f(x+\xi)\}=0, \quad(-\infty<x<\infty),
$$

and let' it be infnitely many time differentiable in any finite interval of $x$-axis.

If, in addition to the Hypothesis of the former,

$$
\varlimsup_{x \rightarrow+\infty} \frac{\log \left|f^{(\nu)}(x)\right|}{x}=\beta_{\nu}, \quad(\nu=0,1,2, \ldots, n),
$$

and

$$
\varlimsup_{x \rightarrow-\infty} \frac{\log \left|f^{(v)}(x)\right|}{x}=\alpha_{r}, \quad(\nu=0,1,2, \ldots, n)
$$

then the coefficients of Cauchy's series of $f(x)$ with respect to the linear transtatable operator $\Gamma$, which correspond to the roots $\lambda_{n}$ such that

$$
\left|R\left(\lambda_{n}\right)\right|>\operatorname{Max}_{0 \leqq \subseteq n}\left(\left|\alpha_{\nu}\right|,\left|\beta_{\nu}\right|\right)
$$

are equal to zero.

Let it be noticed that our theorems may be recognised as a generalisation of the theorems due to Schürer [1] in certain points.

An easy application of Theorem VII. I. will yield us

Theorem VIII. III. Let $f(x)$ be a solution of the functional equation (\%.01) in the interval $\left(x_{0}+\alpha, x_{0}+\beta\right)$, and let it be indefinitely many time differentiable in $\left(x_{0}+a+\alpha, x_{n}+b+\beta\right)\left({ }^{46}\right)$.

If we assume that

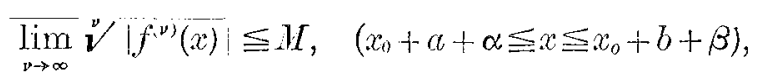

(46) Schürer [1] Satz IX, s. 226, and Satz VII, s. 220-221. See also Theorem XVII. II. Here let it be noticed that, in Schürer theory, it is sufficient that the generating function is defined in certain cir'e. But the modifications of our arguments to contain this general case is not difficult, as we will show in another occasion. 
then the contour-integral $\mathrm{T}_{r}\left(x, x_{0} ; f\right)$ tends to $f(x)$ uniformly in the interval $\left(x_{0}+\alpha+\varepsilon, x_{0}++\beta-\varepsilon\right)$ for any positive number $\varepsilon$ as $r \rightarrow \infty$.

To prove this, let us notice that, for any positive number $\eta$, we have, by the assumption (8.12)

$$
\left|f^{(\nu)}(x)\right| \leqq(M+\eta)^{\nu}, \quad\left(x_{0}+a+\alpha \leqq x_{0} \leqq x_{0}+b+\beta\right) .
$$

If we take $d_{r}$ sufficiently large such that

$$
\frac{M+\eta}{d_{r}}<\frac{1}{2}
$$

then we have, for any choice of the sequence $\{m(r)\}$

$$
\sum_{\nu=0}^{m(r)+n-1} \frac{\left|f^{(\nu)}(x)\right|}{d_{r}^{\nu}}<\sum_{\nu=0}^{\infty}\left(\frac{1}{2}\right)^{\nu}=2 .
$$

Thus one of the assumptions (7.03) in Theorem VII. I. is satisfied. Further, since we may take $m(r)$ as large as we please, we are always able to select $\{m(r)\}$ such that (7.04) may be satisfied.

Therefore we see that the hypothesis in Theorem VII. I. are satisfied, which yields us the proof of our theorem.

\section{§9. The Ritt-Valiron's Theory of Linear of Differential Equation of Infinite Order.}

The expansion-theorems of analytic solutions of the linear differential equation of infinite order in the series of fundamental functions, which are established by Ritt [1] and Valiron [1], were derived from a quite different consideration of the operator $\Gamma$, from those we have developed hitherto. The essential idea of their methods is to approximate the operator $\Lambda$ by the sequence of polynomials of differential operator $P_{r}(D)$ or by the product of the polynomials of differential operators $P_{r}(D)$ and the translation operator $e^{h_{r} D}$; if we denote, by $G_{r}(\lambda)$, the generating function of $r$-th operator of the sequence, then

$$
\begin{aligned}
& P_{r}(D)=c D^{k} \prod_{i=0}^{r}\left(1-\frac{D}{\lambda_{i}}\right), \\
& G_{r}(\lambda)=c \lambda^{k} \prod_{i=0}^{r}\left(1-\frac{\lambda}{\lambda_{i}}\right),
\end{aligned}
$$

in the Ritt's case( ${ }^{47}$ ) and

$$
P_{r}(D)=c D^{k} e^{\beta} r^{D} \prod_{i=0}^{m(r)}\left(1-\frac{D}{\lambda_{i}}\right),
$$

(47) Ritt $[1] \S 5-\S 8$ (p. 36-49). Specially see $\$ 5$. Multiplication and facotrization of operators. 


$$
G_{,}(\lambda)=c \lambda^{k} e^{\beta_{r} \lambda} \prod_{i=0}^{m(r)}\left(1-\frac{\lambda}{\lambda_{i}}\right),
$$

in the Valiron's case ( $\left.{ }^{\text {t8}}\right)$. In both cases we have

$$
\lim _{r \rightarrow \infty} G_{r}(\lambda)=G(\lambda),
$$

where $G(\lambda)$ is the generating function of $\boldsymbol{\Lambda}$.

We may explain their methods as one of the ways to treat our fundamental contour-integrals introduced in the previous chapter, although they did not mention these facts explicitly. Here we should, of course, notice that some modifications to our terminologies and definitions must be performed in order to connect our theory of Cauchy series with these theories. The modifications are necessary in two points. At first the independent rariable should be complex number, and secondly the domain of the operator should consist of infinitely many time differentiable functions, and consequently the continuity-property of the operator should bo defined by another way different from those we have defined in Introduction. Nevertheless these two difficulties are easy to overcome. To define the Cauchy's series for complex variables is a matter of repitation, and as to the continuity of the operator, we have only to notice that our method of estimating tho contourintegral should be replaced by the Ritt-Valiron's method of approximating the operator on the case when $f(z)$ is analytic and $G(\lambda)$ is an integral function of order 0 or order 1 and of certain types.

Let the transmutation be given by

$$
\Lambda f(z)=\sum_{n=0}^{\infty} a_{n} f^{(n)}(z)
$$

where the generating function is

$$
G(\lambda)=\sum_{n=0}^{\infty} a_{n} \lambda^{n}
$$

and let it be an integral function of the order zero or one.

Since this transmutation $\Lambda$ is permutable with differentiation, in our method we should consider the contour-integral defined as in $\$ 1$; that is,

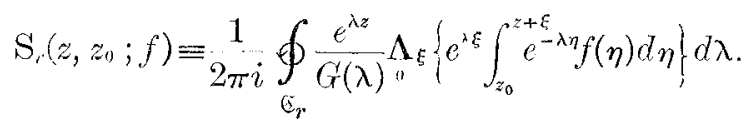

But, in order that we should explain the Ritt-Valiron's method, it may be rather convenient to consider the contour-integral defincd by

$$
\tilde{\mathrm{S}}_{r}\left(z, z_{0} ; f\right) \equiv \frac{1}{2 \pi i} \oint_{\mathfrak{E}_{i},} \frac{1}{G(\lambda)} \Lambda\left(e^{\lambda z} \int_{z_{0}}^{z} e^{-\lambda \eta} f(\eta) d \eta\right) d \lambda .
$$

(4s) Valiron [1], specially $\$ 5$ and $\$ 6 . p .38-43$. Fere we should, of course, mention Pólya [1] on this subject. 
Here we have the following

Theorem. IX. I. If $f(z)$ is an analytic solution of the functional equation

$$
\Lambda f(z) \equiv \sum_{n=0}^{\infty} a_{n} f^{n)}(z)=0, \quad \text { in }\left|z-z_{0}\right| \leqq \rho
$$

then, in the domain $\left|z-z_{1}\right| \leqq \rho$, we have, for any contoui $\mathfrak{C}_{r}$,

$$
\mathrm{S}_{r}\left(z, z_{0} ; f\right)=\tilde{\mathrm{S}}_{r}\left(z, z_{0}: f\right) \text {. }
$$

Proof. By the definitions (9.06) and (9.07), we have

$$
\begin{aligned}
& \mathrm{S}_{r}\left(z, z_{0} ; f\right)-\tilde{\mathrm{S}}_{r}\left(z, z_{0} ; f\right) \\
&=\frac{1}{2 \pi i} \oint_{\xi_{r}} \frac{e^{\lambda z}}{G(\lambda)} \Lambda_{\xi}\left\{e^{\lambda \xi} \int_{z_{0}}^{z_{0}+\xi} e^{-\lambda \eta} f(\eta) d \eta\right\} d \lambda \\
&-\frac{1}{2 \pi i} \oint_{\xi_{r}} \frac{e^{\lambda z}}{G(\lambda)} \Lambda_{\xi}\left\{e^{\lambda \xi} \int_{z_{0}}^{z+\xi} e^{-\lambda \eta} f(\eta) d \eta\right\} d \lambda \\
&= \frac{1}{2 \pi i} \oint_{\xi_{r}} \frac{e^{\lambda z}}{G(\lambda)} \Lambda_{0}\left\{\int_{z}^{z_{0}} e^{-\lambda \xi} f(\xi+\zeta) d \xi\right\} d \lambda \\
&= \frac{1}{2 \pi i} \oint_{\tilde{E}_{r}} \frac{e^{\lambda z}}{G(\lambda)}\left(\int_{z}^{z_{0}} e^{-\lambda \zeta} \Lambda_{\xi}\{f(\xi+\zeta)\} d \zeta\right) d \lambda=0,
\end{aligned}
$$

where, by the assumption (9.08), the last integral is equal to zero, which was to be proved.

This theorem shows us that the series which Ritt, Pólya and Valiron considered and which Valiron called the Dirichlet series of $f(x)$ are idential with our Cauchy's series of $f(x)$ with respect to the operator $\Lambda$.

Now let us define the operator $Q_{r}$, the quotient of $\Lambda$ with respect to $P_{r}$, as follows: the transmutation is given by

$$
\left.Q_{r}(D)=\frac{\Lambda(D)}{P_{r}(D)},{ }^{\text {49 }}\right)
$$

whose generating function is given by

$$
H_{r}(\lambda)=\frac{G(\lambda)}{P_{r}(\lambda)}
$$

which, therefore, is also an integral function of $\lambda$. Then the fundamental facts in the Ritt-Valiron's theory are the following:

1. If $f(z)$ be regular in the domain $\left|z-z_{0}\right|<\sigma+h$, where we assume

(49) This must be unsterstood as the symbolic notation of the operation whose generating function is given by (9.12). Here let it be noticed that, for the analytic function $f(z)$, the operation $\Lambda f(z)$ (if it exist) is uniquely determined if we give the generating function of $\Lambda$. See also Appendix. 


$$
\sigma=\limsup _{|\lambda| \rightarrow \infty} \frac{\log |G(\lambda)|}{|\lambda|}
$$

then, in the circle $\left|z-z_{0}\right|<h(h>0)$, we have

$$
\Lambda g(z)=P_{r}\left\{Q_{r} g(x)\right\}=Q_{r}\left\{P_{r} g(z)\right\} \text {. }
$$

2. In the circle $\left|z-z_{0}\right|<h^{\prime}<h$, we have

$$
\left|Q_{r} g(z)-g\left(z+\beta_{r}\right)\right|<\varepsilon, \quad \text { for } r>N(g, \varepsilon) .
$$

Here let it be noticed that these convenient calculations of the operational equation are justified by the fact that $f(x)$ is analytic in a certain domain. But when $f(x)$ is not analytic, their method is not applicable. This is the reason why we proceed a different course from those of RittValiron's.

\section{Chapter III. Fundamental Theorems of Cauchy's Series.}

\section{$\$ 10$. Linear Translatable Operators to be Considered.}

In the previous chapter we have seen that the convergence-problem of expansion with respect to linear translatable operator depends on the behaviour of the generating function of the operator. According to Theorem III. I, it may be readily recognised that, if we wish to establish a theory of Cauchy's series analogous to that of Fourier's series for the function-set $\left(\mathscr{L}_{0}\right)\left(x_{0}+a, x_{0}+b\right)$, we must restrict ourselves with certain linear translatable operator.

In the sequel of this chapter we shall assume that there is a sequence of contours $\left\{\mathfrak{E}_{r}\right\}$ such that

$$
\left\{\mathfrak{E}_{r}\right\} \varepsilon O(\delta,-\delta)
$$

and further, with respect to this sequence $\left\{\mathfrak{C}_{r}\right\}$,

$$
G(\lambda)=\mathfrak{S}_{r}-O(0 ; a+\delta, b-\delta),
$$

where $\delta$ any given positive number as small as we may please.

The examples of the generating functions which satisfy these conditions (10.01) and (10.02) may bo easily obtained from the examples of $\$ 5$.

An example of the operator whose generating function does not satisfy both (10.01) and (10.02) and therefore such is not the object of this chapter, is given by

$$
\Lambda f(x)=\frac{1}{2} \int_{-1}^{1} f(x+t) d t-f(x) .
$$

In the sequel of this chaper we also assume that the order of the operator $\Lambda$ and the definite integration of any function which is Lebesgueintegrable in any finite interval may be interchanged by Property III. 


\section{$\S 11$. Integration, Uniqueness and Differentiation of Cauchy's Series.}

Let $f(x)$ be a solution of the linear translatable functional equation

$$
\Delta f(x)=0, \quad(-\infty<x<\infty) .
$$

Let the Cauchy's series of $f(x)$ be

$$
f(x) \sim Q_{0}(x)+\sum_{n=1}^{\infty} Q_{n}(x) e^{\lambda} x^{x},
$$

where we assume that the origin is the zero-point of order $m$ of the generating function $\left({ }^{59}\right)$.

The coefficients of the polynomials $Q_{n}(x)$ are, as we have defined in Introduction, determined by the contour-integrals $\mathrm{T}_{r}\left(x, x_{n} ; f\right)$, which is equal to $\mathrm{S}_{r}\left(x, x_{0} ; f\right)$ in this case, and they depend upon the initial point $x_{0}$ in the first appearance.

But in virtue of the fact that $f(x)$ is a solution of the functional equation (11.01), they are in reality independent of $x_{0}$, as is shown in Theorem VI. I.

Now let us put

$$
f_{1}(x)=\int_{a}^{x} f(t) d t-A x^{n}
$$

We will choose a constant $A$ such that we have

$$
\Lambda_{\xi}\left\{f_{1}(x+\xi)\right\}=0 \text {. }
$$

Such $A$ is uniquely determined. For,

$$
\begin{aligned}
\Lambda_{\varepsilon}\{f(x+\xi)\} & =\Lambda_{\varepsilon}\left\{\int_{a}^{x+\xi} f(t) d t-A(x+\xi)^{m}\right\} \\
& =\Lambda_{\varepsilon}\left\{\int_{a+\xi}^{x+\xi} f(t) d t+\int_{a}^{a+\xi} f(t) d t\right\}-A_{0}\left\{\xi^{m}\right\} \\
& =\int_{a}^{x} \Lambda_{0}\{f(\xi+s)\} d s+\Lambda_{\varepsilon}\left\{\int_{a}^{a+\xi} f(t) d t\right\}-A \Lambda_{0}\left\{\xi^{m}\right\} \\
& =\Lambda_{0}\left\{\int_{a}^{a+\xi} f(t) d t\right\}-A \Lambda_{\xi}\left\{\xi^{m}\right\}=0
\end{aligned}
$$

Thus we have

$$
A=\frac{\Lambda_{\xi}\left\{\int_{a}^{a+\xi} f(t) d t\right\}}{\Lambda_{0}\left\{\xi^{m}\right\}},
$$

for

$\left.{ }^{50}\right) m$ is a non-negative integer, and sometimes may be zero. 


$$
\Lambda_{\xi}\left\{\xi^{m}\right\} \neq 0 \text {. }
$$

We will now apply Theorem VI. II. to the function $f_{1}(x)$, which is continuous and of bounded variation in any finite interval, and is a solution of (11.01) in $-\infty<x<\infty$.

Thus we see that the formally defined Cauchy's series of $f_{1}(x)$, that is,

$$
f_{1}(x) \sim \int_{a}^{x} Q_{0}(t) d t+\sum_{n=1}^{\infty} \int_{a}^{x} Q_{n}(t) e^{\lambda_{n} t} d t-A x^{m}
$$

tends to $f_{1}(x)$ uniformly in any finite interval of the $x$-axis.

Thus we have obtained

Theorem XI. I. Let $f^{\prime}(x)$ be L-integrable in any finite interval of the $x$-axis. Let $f(x)$ be a solution of (11.01) with the Cauchy's series (11.02). Then we have

$$
\int_{a}^{x} f(t) d t=\int_{a}^{x} Q_{0}(t) d t+\sum_{n=1}^{\infty} \int_{\alpha}^{x} Q_{n}(t) e^{\lambda} n^{t} d t .
$$

Frurther the convergence in (11.09) is uniform in any finite interval of the $x$-axis.

Now we consider an exponential series defined by

$$
P_{0}(x)+\sum_{n=1}^{\infty} P_{n}(x) e^{\lambda} n^{x},
$$

where $P_{n}(x)(n=0,1,2, \ldots, n, \ldots)$ are arbitrary polynomials whose degree are less than the multiplicities of $\lambda_{n}$ as the roots of

$$
G(\lambda)=0 \text {, }
$$

$G(\lambda)$ being of course the generating function of the operator $\Lambda$.

As the immediate consequence of this theorem we get

Theorem XI. II. The necessary and sufficient condition that an exponcntial series with respect to a linear translatable operator given by (11.10) should be the Cauchy's series of a L-integrable function is that the series

$$
\int_{a}^{x} P_{0}(t) d t+\sum_{t=1}^{\infty} \int_{a}^{x} P_{n}(t) e^{\lambda} n^{t} d t
$$

converges to an indefinite integral, uniformly in any fnite interval.

Further we obtain

Theorem XI. III. Let $f(x)$ and $h(x)$ be solutions of the functional equation (11.01). If we assume that these two functions are L-integrable in any finite interval and have the same Cauchy's series, then we have, for almost all $x$,

$$
f(x)=h(x) .
$$


The convergence-theorem in $\$ 3$ gives us the following

Theorem XI. IV. Let us assume that $f(x)$ is continuous in $\left(x_{0}+a, x_{0}+b\right)$ and $\left(\mathscr{L}_{n}\right)\left(x_{0}+a, x_{0}+b\right)$. Further let

$$
B_{m}\left(f, x_{0}\right)=0 .
$$
that is

Then the Cauchy-series of $f^{(m)}(x)$ is obtained by termwise differentiation,

$$
f^{(m)}(x) \sim \frac{d^{m}}{d x^{m}}\left\{P_{0}(x) e^{\lambda_{0} x}\right\}+\sum_{n=1}^{\infty} \frac{d^{m}}{d x^{m}}\left\{P_{n}(x) e^{\lambda_{n} n^{x}}\right\}
$$

\section{$\S$ 12. A Certain Generalisation of Parseval's Theorem.}

The assumptions $(\mathbf{1 0 . 0 1 )}$ and (10.02) give us, in view of the consequence in $\$ 2$,

$$
\mathrm{S}_{r}\left(x, x_{0} ; f\right)=\frac{1}{\pi} \int_{x_{0}+a}^{x_{0}+b} \frac{\sin \rho_{r}(x-\eta)}{x-\eta} f(\eta) d \eta+\varepsilon_{r}\left(x, x_{\theta} ; f\right),
$$

where $\varepsilon_{r}\left(x, x_{0} ; f\right)$ tends to zero as $r \rightarrow \infty$, for the sequence $\{\mathfrak{S}$,$\} satisfies$ (10.01), uniformly concerning $x$ in the intervai $\left(x_{0}+a+\frac{\delta}{2}, x_{0}+b-\frac{\delta}{2}\right), \delta$ being any given positive number.

Let us now introduce the Feje'r-sum of Cauchy's series, which is defined by

$$
\sigma_{n}\left(x, x_{0} ; f\right)=\frac{1}{n} \sum_{r=0}^{n-1} \mathrm{~S}_{r}\left(x, x_{0} ; f\right)
$$

By (12.01) we have

$$
\begin{aligned}
\sigma_{n}\left(x, x_{0} ; f\right)= & \frac{1}{n \pi} \int_{x_{0}+a}^{x_{0}+b} \frac{\sum_{r=0}^{n-1} \sin \rho_{r} \cdot(x-\eta)}{x-\eta} f(\eta) d \eta \\
& +\frac{1}{n} \sum_{r=0}^{n-1} \varepsilon_{r}\left(x, x_{0} ; f\right),
\end{aligned}
$$

Hereafter in this paragraph we put $a=-\pi, b=\pi$ for the sake of simplicity, and we assume that the following condition is satisfied:

Condition $3^{\circ}$. For the value $t$ in the interval $-\pi \leqq t \leqq \pi$, we have

$$
\frac{1}{n}\left\{\sum_{r=1}^{n-1} \sin \rho_{r} t-\sum_{r=0}^{n-1} \sin (r+1) t\right\}=0(1), \quad(\text { as } n \rightarrow \infty)
$$

This assumption gives us

$$
\begin{aligned}
\sigma_{n}\left(x, x_{0} ; f\right)= & \frac{1}{2 n \pi} \int_{x_{0}-\pi}^{x_{0}+\pi} f(\eta) \frac{\sum_{r=0}^{n-1} \sin (r+1)(x-\eta)}{\frac{x-\eta}{2}} d \eta \\
& +\varepsilon_{n}^{\prime}\left(x, x_{n} ; f\right),
\end{aligned}
$$


where $\varepsilon_{n}^{\prime}\left(x, x_{0} ; f\right)$ tends to zero as $x \rightarrow \infty$, uniformly concerning $x$ in the interval $\left(x_{0}-\pi+\delta, x_{0}+\pi-\delta\right), \delta$ being any given positive number.

(12.05) may also be written as follows:

$$
\sigma_{n}\left(x, x_{0} ; f\right)=\frac{1}{2 n \pi} \int_{x_{0}-\pi}^{x_{0}+\pi} f(\eta)\left(\frac{\sin \frac{n(x-\eta)}{2}}{\frac{x-\eta}{2}}\right)^{2} d \eta+\varepsilon_{n}\left(x, x_{0} ; f\right),
$$

where $\varepsilon_{n}\left(x, x_{0} ; f\right)$ has the same property as $\varepsilon_{n}^{\prime}\left(x, x_{0} ; f\right)$.

Now (12.06) leads us to a generalisation of the theorem due to $F$. Riesz $^{(51)}$ :

Theorem XII. I. If $f(x)$ belongs to the class $L^{p}\left(x_{0}-\pi, x_{0}+\pi\right)(p>1)$, then we have

$$
\lim _{n \rightarrow \infty} \int_{x_{0}-\pi}^{x_{0}+\pi}\left|f(t)-\mathrm{S}_{n}\left(t, x_{0} ; f\right)\right|^{p} d t=0 .
$$

Indeed, we have only to repeat the argument in the proof of M. Riesz's theorem concerning the Fourier's series.

To generalise the Parseval's theorem of Fourier's series to our Cauchy's series, we will prepare ourselves with

Definition VI. Under the pseudo-inner product $(f, g)$ we understand the real or complex number defined by

$$
(f, g)=\mathbf{\Lambda}_{0}\left\{\int_{0}^{t} f(\eta) \overline{g(t-\eta)} d \eta\right\} .
$$

Due to the definition, pseudo-inner products have the following properties:

$$
\begin{cases}1^{\circ} . & (c f+d h, g)=c(f, g)+d(h, g), \\ 2^{\circ} . & (f, g)==(g, f),\end{cases}
$$

where $c$ and $d$ are any real or complex numbers.

We will express the pseudo-inner product $(f, g)$ by the coefficients of expansion of $f(x)$ and $g(x)$.

For this purpose we assume furthermore

Condition $4^{\circ}$. The generating function $G(\lambda)$ has no multiple roots, and for any complex number $\lambda$, we have

$$
\overline{G(\lambda)}=G(\lambda)
$$

If Condition $4^{\circ}$ is satisfied, then we may change the summation from 0 to $\infty$ in (11.10) into the summation from $-\infty$ to $\infty$, and we may-and we shall write

(51) Riesz, F. [1] and [2]. Specially See Riesz, F. [2] Quelques applications à sérié de Fourier. $\$ 13-\$ 16 .($ p. $230-232)$. 


$$
f(x) \sim \sum_{k=-\infty}^{\infty} a_{k} e^{\lambda} k^{x}
$$

and

$$
g(x) \sim \sum_{k=-\infty}^{\infty} b_{k} e^{\lambda} k^{n}
$$

assuming

(12.13)

$$
\bar{\lambda}_{k}=\lambda_{-k}, \quad(k=1,2,3, \ldots)
$$

For the sake of brevity we introduce

Definition VIl. The generating function which satisfies the condition (10.01), (10.12), Condition $3^{\circ}$ and Condition $4^{\circ}$ is called to be of complete type.

After these preparations we get

Theorem XII. II. $\left({ }^{52}\right)$ Let $f(x)$ and $g(x)$ be solutions of the functional equation (11.01) and let the generating function $G_{1}(\lambda)$ of $\Lambda_{2}$ given in $\$ 5$

$$
G_{i}(\lambda)=\int_{-\pi-0}^{\pi+i t} e^{\lambda t} d \varphi(t)
$$

be of complete type. Further let us assume that $f(x)$ and $g(x)$ belong to $L^{p}(2 a, 2 b)$ and $L^{q}(2 \alpha, 2 b)$ respectively, where

$$
\frac{1}{p}+\frac{1}{q}=1
$$

and that

$$
\int_{\eta-0}^{\pi+0} g(t-\eta) d \varphi(t), \quad(-\pi \leqq \eta \leqq \pi)
$$

belongs to $L^{q}(2 a, 2 b)$.

Under these conditions, let it be assumed that the pseudo-inner product, $(f, g)$ may be expressed

$$
(f, g)=\int_{-\pi+0}^{\pi} f(\eta)\left(\int_{\eta-0}^{\pi+\frac{\theta}{g(t-\eta)}} d \varphi(t)\right) d \eta .
$$

Then we obtain

$$
(f, g)=\lim _{n \rightarrow \infty} \sum_{k=-n}^{n} a_{k} \bar{b}_{-k} G^{\prime}\left(\lambda_{k}\right)
$$

Proof. Let us write, for a moment,

$$
f_{n}(x)=\sum_{k=-n}^{n} a_{k} e^{\lambda_{k} x}
$$

Then $f_{n}(x)$ satisfies the same conditions which $f(x)$ satisfies, that is, $f_{n}(x)$ is a solution of the functional equation (11.01) and belongs to $L^{p}(2 a, 2 b)$.

(52) Some of the assumptions in this theorem will be perhaps superfluous. Elabolations are left for further researches. 
Therefore, by the assumption (12.17), we may write

$$
(f, g)-\left(f_{n}, g\right)=\int_{-\pi}^{\pi}\left\{f(\eta)-f_{n}(\eta)\right\}\left\{\int_{\eta-0}^{\pi+0} \overline{g(t-\eta)} d \varphi(t)\right\} d \eta,
$$

which yields us, in view of our assumption that $f(x)$ and $g(x)$ belong to $L^{p}(2 a, 2 b)$ and $L^{q}(2 a, 2 b)$ respectively,

$$
\text { (12.21) }\left|(f, g)-\left(f_{n}, g\right)\right| \leqq \int_{-\pi}^{\pi}\left|f(\eta)-f_{n}(\eta)\right|^{p} d \eta \int_{-\pi}^{\pi}\left|\int_{\eta-0}^{\pi+0} \overline{g(t-\eta)} d \varphi(t)\right|^{i q} d \eta \text {. }
$$

Applying Theorem XII.I, we obtain

$$
\lim _{n \rightarrow \infty}\left(f_{n}, g\right)=(f, g) \text {. }
$$

But since, as easily may be verified,

$$
\left(f_{n}, g\right)=\sum_{k=-n}^{n} a_{k} \bar{b}_{-k} G^{\prime}\left(\lambda_{k}\right)
$$

we obtain

$(12.24)$

$$
(f, g)=\lim _{n \rightarrow \infty} \sum_{k=-n}^{n} a_{k} \bar{b}_{-k} G^{\prime}\left(\lambda_{k}\right)
$$

which was to be proved,

Example 1. In the Fourier series, we may put

$$
\begin{aligned}
\varphi(t) & =0, & & (-\infty<t<-\pi) . \\
& =-1, & & (-\pi \leqq t \leqq \pi) . \\
& =0, & & (\pi<t<\infty) .
\end{aligned}
$$

Hence if we assume that $g(t)$ is a solution of the functional equation allied to this operator $\Lambda_{2} f(x) \equiv f(x+\pi)-f(x-\pi)$, that is to say,

$$
g(t)=g(t+2 \pi), \quad(-\infty<t<\infty)
$$

then we obtain

$$
\begin{aligned}
& \int_{-\pi-0}^{\pi+0}\left(\int_{0}^{t} g(\eta) \overline{f(t-\eta)} d \eta\right) d \varphi(t) \\
& \quad=-\int_{0}^{\pi} f(\eta) \overline{g(-\pi-\eta)} d \eta+\int_{0}^{\pi} f(\eta \overline{g(\pi-\eta)} d \eta \\
& \quad=\int_{-\pi}^{\pi} f(\eta) \overline{g(\pi-\eta)} d \eta .
\end{aligned}
$$

On the other hand we may write

$$
\begin{aligned}
& \int_{-\pi}^{\pi} f(\eta)\left(\int_{\eta-0}^{\pi+\frac{+0}{g(t-\eta)}} d \varphi(t)\right) d \eta \\
& \left.\quad=-\int_{-\pi}^{\pi} f(\eta) \overline{g(-\pi-\eta)} d \eta+\int_{-\pi}^{\pi} f(\eta) \overline{g(\pi-\eta)} d \eta+\int_{-\pi}^{\pi} f(\eta) \overline{g(\pi-\eta}\right) d \eta
\end{aligned}
$$




$$
=\int_{-\pi}^{\pi} f(\eta) \overline{g(\pi-\eta)} d \eta
$$

Therefore we see that the assumption (12.17) in Theorem XII. II. is satisfied. Further let it be noticed that

$$
\begin{aligned}
c_{k} & =\frac{1}{G^{\prime}\left(\lambda_{k}\right)} \int_{-\pi-0}^{\pi+0} e^{\lambda_{l^{t}}}\left(\int_{0}^{t} e^{-\lambda_{k} \eta} f(\eta) d \eta\right) d \varphi(t) \\
& =\frac{1}{G^{\prime}(i k)} \int_{-\pi}^{\pi} f(\eta) e^{-i k(\pi-\eta)} d \eta=\frac{1}{2 \pi} \int_{-\pi}^{\pi} f(\eta) e^{i k \eta} d \eta .
\end{aligned}
$$

Consequently if we put

$$
g(t) \equiv f(\pi-t)
$$

then we have

(12.31)

$$
b_{-k}=\frac{e^{-i k \pi}}{2 \pi} \int_{-\pi}^{\pi} f(\zeta) e^{i k \zeta} d \zeta
$$

and

$$
\sum_{k=-n}^{n} a_{k} \bar{b}_{-k} G^{\prime}\left(\lambda_{k}\right)=\frac{1}{2 \pi} \sum_{k=-n}^{n}\left|\int_{\pi}^{\pi} f(\zeta) e^{i k \zeta} d \zeta\right|^{2}
$$

Combinating (12.24) (12.27), (12.28) and (12.30), we reach

$$
\begin{aligned}
\Lambda_{1, t}\left\{\int_{0}^{t} f(\eta) \overline{f(\pi-t+\eta)} d \eta\right\} & =\int_{-\pi}^{\pi}|f(\eta)|^{2} d \eta \\
& =\lim _{n \rightarrow \infty}-\frac{1}{2 \pi} \sum_{k=-n}^{n} \mid \int_{-\pi}^{\pi} f(\zeta) e^{i k \zeta} d \zeta^{2}
\end{aligned}
$$

which is nothing but the Parseval's relation.

We finish this paragraph, giving the following property of pseudo-inner product which we number in

Theorem XII. III. If $f(x)$ and $g(x)$ are solutions of the functional equation (11.01), then the pseudo-inner product $(f, g)$ is invariant under the translations of $f(x)$ and $g(x)$; that is, if we write

$$
\begin{aligned}
& \mathrm{T}_{\sigma} f(x) \equiv f(x+\sigma) \equiv f_{\sigma}(x), \\
& \mathrm{T}_{\rho} g(x) \equiv g(x+\rho) \equiv g_{\rho}(x),
\end{aligned}
$$

then we have, for any real numbers $\sigma$ and $\rho$,

$$
\left(f_{\sigma}, g_{\rho}\right)=(f, g) \text {. }
$$

To prove this, let us calculate the difference

$$
\left(f_{\sigma}, g\right)-(f, g) \text {. }
$$

By the similar argument to that of (6.08), this may be written

$$
\begin{aligned}
& \left(f_{\sigma}, g\right)-(f, g) \\
& =\Lambda_{0}\left\{\int_{t}^{\sigma+t} f(\eta) \overline{g(t-\eta)} d \eta\right\}-\Lambda_{0}\left\{\int_{0}^{\sigma} f(\eta) \overline{g(t-\eta)} d \eta\right\}
\end{aligned}
$$




$$
=\int_{0}^{\sigma} \Lambda_{0}\{f(t+\eta)\} g(-\eta) d \eta-\int_{0}^{\sigma} f(\eta) \Lambda_{0}\{\overline{g(t-\eta)}\} d \eta
$$

Since $f(x)$ and $g(x)$ are solutions of (11.01), the last two integrands are equal to zero. Thus we have, for any $\sigma$,

$$
\left(f_{\sigma}, g\right)=(f, g) \text {. }
$$

Let $\sigma$ and $\rho$ be any given real numbers. We have

$$
\begin{aligned}
\left(f_{\sigma}, g_{\rho}\right) & =\left(f, g_{\rho}\right), \quad \text { by }(12.39), \\
& =\left(\overline{g_{\rho}, f}\right), \quad \text { by } \quad(12.09) 2^{\circ}, \\
& =\overline{(g, f),} \quad \text { by } \cdot(12.39), \\
& =(f, g), \quad \text { by } \quad(12.09) 2^{\circ},
\end{aligned}
$$

which we were to prove.

\section{$\S 13$. Multiplication-theorems.}

In this section we will mention some multiplication-theorems under the stronger assumption. The condition is that the Cauchy's series of the functions in consideration converge uniformly in a certain interval of the length greater than $2(b-a)$. Then the formal calculations are permitted, so that we may easily obtain the following theorem:

Theorems. IX. I. Let us assume that the series

$$
f(x)=\sum_{k=0}^{\infty}\left(\sum_{\nu=\omega}^{p_{k}-1} \alpha_{k, v} \cdot x^{\nu}\right) e^{\lambda k^{x}},
$$

and

$$
g(x)=\sum_{k=0}^{\infty}\left(\sum_{\nu=0}^{p_{k}-1} b_{k, \nu} x^{\nu}\right) e^{\lambda_{k} x}
$$

are uniformly convergent in the interval $x_{0}+a \leqq x \leqq x_{0}+b$.

Then

$$
\begin{aligned}
& \Lambda_{0}\left\{\int_{x_{0}}^{x_{0}+t} f(\eta) g(t-\eta) d \eta\right\} \\
& =\sum_{k=0}^{\infty}\left\{\sum_{\nu=0}^{p_{k}-1} b_{k, \nu} \nu ! \sum_{s=p_{k}=\nu-1}^{n_{k} k^{-1}} \alpha_{k_{i}}, \frac{s !}{(s+\nu+1) !} G^{(s+\nu+1)}\left(\lambda_{k}\right)\right\} .
\end{aligned}
$$

Remark 1. When specially the generating function $G(\lambda)$ has no multiple roots, then (13.03) becomes

$$
\Lambda_{0}\left\{\int_{x_{0}}^{x_{0}+t} f(\eta) g(t-\eta) d \eta\right\}=\sum_{k=0}^{\infty} a_{k} b_{k} G^{\prime}\left(\lambda_{k}\right)
$$

Remark 2. When specially the generating function has no multiple 
roots and $f(x)$ and $g(x)$ are solutions of the linear translatable equation, then

(13.05) $\Lambda_{0}\left\{\int_{x_{0}}^{x_{0}+t} f(x+\eta) g(y+t-\eta) d \eta\right\}=\sum_{k=0}^{\infty} a_{k} b_{k} G^{\prime}\left(\lambda_{k}\right) e^{\lambda_{k}(x+y)}$, where $\left\{a_{k}\right\}$ and $\left\{b_{k}\right\}$ are independent upon $x_{0}$.

\section{$\S 14$. Integral-representation of the Biorthogonal Functions.}

In this section we assume that the generating function is of complete type.

Let the expansion of $f(x)$ be

$$
f(x) \sim \sum_{k=-\infty}^{\infty} \alpha_{k} e^{\lambda} k^{x}
$$

Then by the contour-integral, we have

$$
a_{n}=\frac{1}{G^{\prime}\left(\lambda_{n}\right)} \int_{a-0}^{b+0}\left\{\int_{0}^{\varepsilon} e^{\lambda_{n}(\xi-\eta)} f(\eta) d \eta\right\} d \varphi(\xi) .
$$

Let $f(x)$ be a solution of the linear translatable functional equation. Then we have, as in (12.17),

$$
a_{n}=\frac{1}{G^{\prime}\left(\lambda_{t}\right)} \int_{a}^{b} f(\eta) e^{-\lambda_{n} \eta}\left(\int_{\eta-0}^{b+0} e^{\lambda} n^{\xi} d \varphi(\xi)\right) d \eta
$$

Putting

$$
h_{n}(\eta)=\frac{1}{G^{\prime}\left(\lambda_{n}\right)} \int_{\eta \rightarrow 0}^{b+0} e^{\lambda} n^{\xi} d \varphi(\xi)
$$

it is readily to be seen

$$
\begin{aligned}
\int_{\alpha}^{b} e^{-\lambda_{m} \eta} h_{n}(\eta) d \eta & =1, \quad m=n \\
& =0, \quad m \neq n
\end{aligned}
$$

so that $\left\{e^{-\lambda_{m} \eta}\right\}$ and $\left\{h_{m}(\eta)\right\}$ form the biorthogonal function-system.

Now we will express $h_{n}(\eta)$ by Laplace-integral.

Let us put

$$
\begin{aligned}
A(\lambda, \eta) & =e^{-\lambda \eta} \int_{\eta-0}^{b+0} e^{\lambda t} d \varphi(t), & & a \leqq \eta \leqq b . \\
& =0, & & \eta<a . \\
& =0, & & \eta>b .
\end{aligned}
$$

and

$$
f(z, \lambda)=\int_{-\infty}^{\infty} e^{z \eta} d_{\eta} A(\lambda, \eta) .
$$

Specially when $\lambda$ is a zero-point of the generating function $G(\lambda)$, then, after simple calculations, we obtain 


$$
f(z, \lambda)=\frac{z}{\lambda-z} G(z) .
$$

The theory of Laplace-integral gives us

$$
\frac{1}{2 \pi i} \lim _{r \rightarrow \infty} \int_{-i T}^{i T} \frac{f(z, \lambda)}{z} e^{u z} d z=\frac{1}{2}\{A(u+0, \lambda)+A(u-0, \lambda)\},
$$

accordingly we have

$$
\frac{1}{2}\{A(u+0, \lambda)+A(u-0, \lambda)\}=\frac{1}{2 \pi i} \lim _{T \rightarrow \infty} \int_{-i T}^{i T} \frac{G(z)}{\lambda-z} e^{u z} d z,
$$

which yields us

$$
\frac{h_{k}(\eta+0)+h_{k}(\eta-0)}{2}=\frac{1}{2 \pi i G^{\prime}\left(\lambda_{k}\right)} \lim _{T \rightarrow \infty} \int_{-i T}^{i r} \frac{G(z)}{\lambda_{k i}-z} e^{u z} d z .
$$

\section{Chapter IV. General Linear Translatable Functional Equations $\left(\boldsymbol{S}^{* \sigma}\right)$ and $\left(\boldsymbol{S}_{\sigma_{1}}^{* * \sigma_{2}}\right)$.}

\$15. Transformation of Contour-Integrals with respect to the Linear Translatable Differential Operators $\Gamma^{*} f(x)$ and $\Gamma^{* *} f(x)$.

1. First let us consider the contour-integral

$$
\mathrm{S}_{. A}^{*}\left(x, \varkappa_{0} ; f\right)=\frac{1}{2 \pi i} \oint_{\mathfrak{E}_{\Lambda}} \frac{e^{\lambda x}}{P(\lambda) G^{*}(\lambda)} \sum_{k=0}^{n} \hat{\Lambda}_{\xi}^{k}\left\{e^{\lambda \xi} \int_{x_{0}}^{x 0+\xi \xi} e^{-\lambda \eta} f^{(k)}(\eta) d \eta\right\} d \lambda,
$$

where the generating functions $G_{k}^{*}(\lambda)$ of the linear translatable operators $\Lambda^{* k} f$, and therefore $G^{*}(\lambda)$ are all regular in a half-plane $R(\lambda)<\sigma$, the operators $\Lambda^{* k} f$ being those which we have defined in Introduction ${ }^{(53)}$.

The concurrence-function $f(x)$ is subject to the following:

Assumption I. Let $f(x)$ be at least n-time differentiable, and let

$$
\int_{0}^{\infty}\left|f^{(k)}(x)\right|^{n}(1+x) e^{-\beta x} d x<\infty, \quad\left(\begin{array}{l}
p=1,2 \\
k=0,1,2, \ldots, n
\end{array}\right)
$$

where $\beta$ is subject to $\beta<\sigma$.

Assumption II. For the same number $\beta$ as in (15.02), let

$$
f^{(k)}(x)=o\left(e^{\beta x}\right), \quad(k=0,1,2, \ldots, n)
$$

Let us suppose that the contour $\mathbb{C}_{A}$ passes through the points $\beta-i A$, $\beta+i A, \gamma+i A$ and $\gamma-i A$ in the counter-clockwise sense, the parts of $\mathfrak{E}_{A}$ between these four points in the above order being denoted by $\mathfrak{E}_{A}^{(i)}(i=1,2,3,4)$ respectively.

Denoting by $\mathfrak{E}_{\xi+i u}^{\xi+i n}$ the segment which is parallel to the imaginary axis and which runs from $\xi+i u$ to $\xi+i v$, we make a further assumption that $\mathbb{S}_{-1}^{(1)}$ is identical with $\underset{\beta-i \Delta}{\beta+i A}$.

$\left.{ }^{53}\right)$ The choice of the convergence-factor $P(\lambda)$ will be determined afterwards. 
We will now decompose the integral (15.01) into four parts:

$$
\begin{aligned}
\mathrm{S}_{A}^{*}\left(x, x_{0} ; f\right) & =\frac{1}{2 \pi i}\left\{\int_{\mathfrak{C}_{A}^{(1)}}+\int_{\mathbb{C}_{A}^{(2)}}+\int_{\mathbb{C}_{A}^{(3)}}+\int_{\mathfrak{C}_{A}^{(4)}}\right\} \\
& =\mathrm{S}_{A, 1}^{*}+\mathrm{S}_{A, 2}^{*}+\mathrm{S}_{A, 3}^{*}+\mathrm{S}_{A, 4}^{*},
\end{aligned}
$$

say. In view of (15.02) and (15.03), we obtain

$$
\begin{aligned}
\int_{x_{0}}^{x_{0}+\xi} e^{-\lambda \eta} f^{(k)}(\eta) d \eta=\int_{x_{0}}^{\infty} e^{-\lambda \eta} f^{(k)}(\eta) d \eta-\int_{x_{0}+\xi}^{\infty} e^{-\lambda \eta} f^{(k)}(\eta) d \eta & \\
\sum_{k=0}^{n} \hat{\Lambda}_{0}^{*}\left\{e^{\lambda \xi} \int_{x_{0}}^{x_{0}+\xi} e^{-\lambda \eta} f^{(k)}(\eta) d \eta\right\}= & \int_{x_{0}}^{\infty} e^{-\lambda \eta} f(\eta) d \eta-\sum_{k=1}^{n} \lambda^{k} G_{\bar{k}}^{*}(\lambda) \sum_{\nu=0}^{k-1} \frac{f^{(\nu)}\left(x_{u}\right)}{\lambda^{\nu+1}} \\
& -\int_{x_{0}+\xi}^{\infty} e^{-\lambda \eta} f^{(k)}(\eta) d \eta, \quad(k=1,2, \ldots, n) .
\end{aligned}
$$

Substituting this into $\mathrm{S}_{\hat{4}, 1}$, we have, after some clementary calculations as in (1.05),

$$
\begin{aligned}
& \mathrm{S}_{A, 1}^{*}=\frac{1}{2 \pi} \int_{-, A}^{\Lambda} \frac{e^{(\beta+i t) x}}{P(\beta+i t)}\left\{\int_{x_{0}}^{\infty} e^{-(\beta+i t) \eta} f(\eta) d \eta\right\} d t \\
& -\frac{1}{2 \pi i} \int_{\mathbb{C}_{1}^{(1)}} \frac{e^{\lambda x}}{P(\lambda) G^{*}(\lambda)}\left\{\sum_{k=1}^{n} \lambda^{k} G_{k}^{*}(\lambda) \sum_{\nu=0}^{k-1} \frac{f^{(\nu)}\left(x_{0}\right)}{\lambda^{\nu+1}}\right\} d \lambda
\end{aligned}
$$

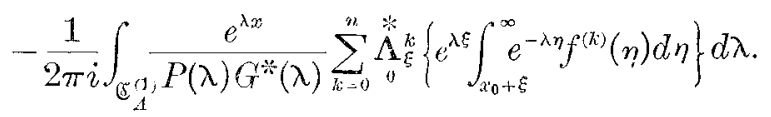

The integration by perts as in (1.04) yields us

$$
\begin{array}{r}
\int_{0_{0}}^{x_{0}+\xi} e^{-\lambda \eta} f^{(k)}(\eta) d \eta=\lambda^{k} \int_{x_{0}}^{x_{0}+\xi} e^{-\lambda \eta} f(\eta) d \eta+\sum_{\nu \sim 0}^{k-1} \lambda^{k-1-\nu}\left[f^{(\nu)}(\eta) e^{-\lambda \eta}\right]_{x_{0}}^{x_{0}+\xi}, \\
(k=1,2, \ldots, n),
\end{array}
$$

and therefore we reach

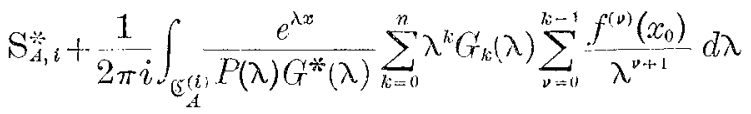

$$
\begin{aligned}
& =\frac{1}{2 \pi i} \int_{\mathscr{C}_{A}^{(i)}} \frac{e^{\lambda x}}{P(\lambda) G^{*}(\lambda)} \sum_{k=0}^{n} \tilde{\Lambda}_{\sigma}^{k}\left\{e^{\lambda \xi} \int_{x_{0}}^{x_{0}+\xi} e^{-\lambda \eta} f(\eta) d \eta\right\} d \lambda
\end{aligned}
$$

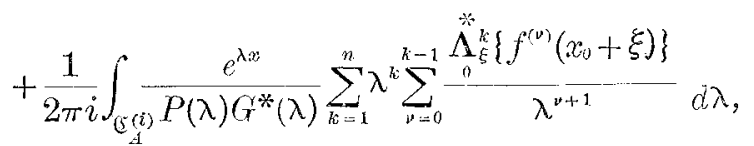

$$
\begin{aligned}
& (i=2,3,4) \text {. }
\end{aligned}
$$

As to the integral $\mathrm{S}_{\pi_{3},}^{*}$, we notice that 
(15.09)

$$
\begin{aligned}
& \frac{1}{2 \pi i} \int_{\mathbb{S}_{A}^{(3)}} \frac{e^{\lambda x}}{P(\lambda) G^{*}(\lambda)} \sum_{k=0}^{n} \lambda^{\lambda_{0}} \hat{\Lambda}_{i}^{*}\left\{e^{\lambda \xi} \int_{x_{0}}^{x_{0}+\xi} e^{-\lambda \eta} f(\eta) d \eta\right\} d \lambda
\end{aligned}
$$

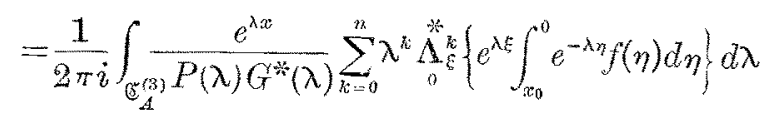

$$
\begin{aligned}
& +\frac{1}{2 \pi i} \int_{\mathbb{C}^{(3)}} \frac{e^{\lambda x}}{P(\lambda) G^{*}}(\lambda) \sum_{i=0}^{n} \lambda^{\hbar} \hat{\Lambda}_{\theta}^{*}\left\{e^{\lambda \xi} \int_{0}^{x_{0}+\xi} e^{-\lambda \eta} f(\eta) d \eta\right\} d \lambda,
\end{aligned}
$$

where, denoting by $\mathfrak{E}_{A}^{(3)}+\mathfrak{C}_{\gamma-i d}^{\gamma+i A}$ the counter-clockwise oriented contour composed with $\mathbb{C}_{4}^{(3)}$ and $\mathcal{E}_{\gamma-i A}^{\gamma+i A}$, the first term of the right-hand side becomes

$$
\begin{aligned}
& \frac{1}{2 \pi i} \int_{\mathbb{C}_{A}^{(3)}} \frac{e^{\lambda x}}{\quad P(\lambda) G^{*}(\lambda)} \sum_{i=0}^{n} \lambda^{k} \Lambda_{\hat{\theta}}^{*}\left\{e^{\lambda \xi} \int_{x_{0}}^{0} e^{-\lambda \eta} f(\eta) d \eta\right\} d \lambda \\
& =\frac{1}{2 \pi i} \oint \frac{e^{\lambda \cdot x}}{P(\lambda)}\left\{\int_{x_{0}}^{0} e^{-\lambda \eta} f(\eta) d \eta\right\} d \lambda \\
& \quad+\frac{1}{2 \pi i} \int_{A}^{-A} \frac{e^{(\gamma+i t) x}}{P(\gamma+i t)}\left\{\int_{x_{0}}^{0} e^{-(i+i t) \eta} f(\eta) d \eta\right\} d t .
\end{aligned}
$$

Therefore, if we put

$$
\text { (15.11) } \mathrm{T}_{\Delta}^{*}\left(x, x_{0} ; f\right)=\frac{1}{2 \pi i} \oint_{\mathbb{E}_{1}} \frac{e^{\lambda x}}{P(\lambda) G^{*}(\lambda)} K^{*}\left(\lambda, x_{0} ; f\right) d \lambda,
$$

where $K^{*}\left(\lambda, x_{0} ; f\right)$ has the same form as $K\left(\lambda, x_{n} ; f\right)$ in $\$ 6$ with respect to $\Gamma^{*} f$, then we have, in view of (15.01) and (15.08)-(15.10),

(15.12) $\quad \mathrm{T}_{A}^{*}\left(x, x_{0} ; f\right)$

$$
\begin{aligned}
& =\frac{1}{2 \pi} \int_{-A}^{A} \frac{e^{\langle\beta+i t): x}}{P(\beta+i t)}\left\{\int_{x_{0}}^{\infty} e^{-(\beta+i t) \eta} f(\eta) d \eta\right\} d t \\
& +\frac{1}{2 \pi} \int_{A}^{-4} \frac{e^{(\gamma+i t) x}}{P(\gamma+i t)}\left\{\int_{x_{0}}^{0} e^{-(\gamma+i t) \eta} f(\eta) d \eta\right\} d t \\
& -\frac{1}{2 \pi}-\int_{-\alpha}^{A} \frac{e^{(\beta+i t) x}}{P(\beta+i t) G(\beta+i t)} \sum_{i=0=0}^{n} \hat{\Lambda}_{\theta}^{*}\left\{e^{(\beta+i t) \xi} \int_{x_{0}+\xi}^{\infty} e^{-(\beta+i t) \eta} f(\eta) d \eta\right\} d \lambda \\
& +\frac{1}{2 \pi i} \oint \frac{e^{\lambda x}}{P(\lambda)}\left\{\int_{x_{0}}^{0} e^{-\lambda \eta} f(\eta) d \eta\right\} d \lambda \\
& \mathrm{C}_{A}^{(3)}+\mathrm{C}_{\gamma-i \mathrm{~A}}^{\mathrm{y}+\mathrm{i}} \\
& +\frac{1}{2 \pi i} \sum_{i=2}^{4} \int_{\mathbb{C}_{A}^{(i)}} \frac{e^{\lambda, x}}{\left.P_{(} \lambda\right) G^{*}(\lambda)} \sum_{k=1}^{n} \lambda^{k} \sum_{p=0}^{k-1} \frac{\hat{\Lambda}_{0}^{k}\left\{f^{(\nu)}\left(x_{0}+\xi\right)\right\}}{\lambda^{p+1}} d \lambda
\end{aligned}
$$

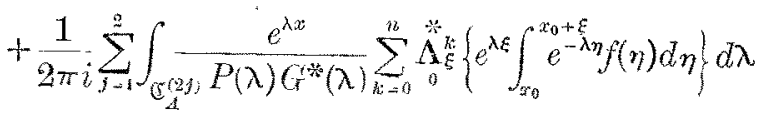

$$
\begin{aligned}
& +\frac{1}{2 \pi i} \int_{\mathfrak{E}^{(3)}} \frac{e^{\lambda x}}{P(\lambda) G^{*}(\lambda)} \sum_{i=4)}^{n} \lambda^{k} \tilde{\Lambda}_{\xi}^{*}\left\{e^{\lambda \xi} \int_{0}^{x_{0}+\xi} e^{-\lambda \eta} f(\eta) d \eta\right\} d \lambda \text {. }
\end{aligned}
$$


2. Next let us consider the contour-integral

$$
\mathrm{S}_{A}^{* *}\left(x, x_{0} ; f\right)=\frac{1}{2 \pi i} \oint_{\mathscr{E}_{-}} \frac{e^{\lambda x}}{P(\lambda) G^{* * *}(\lambda)} \sum_{k=0}^{n} \hat{\Lambda}_{\xi}^{*}\left\{e^{\lambda \xi} \int_{x_{0}}^{x_{0}+\xi} e^{-\lambda \eta} f^{(k)}(\eta) d \eta\right\} d \lambda,
$$

where the generating functions $G_{*}^{* *}(\lambda)$ of the linear translatable operators $\Lambda^{* * k}$ and hence $G^{* *}(\lambda)$ are all regular in a strip $\sigma_{1}<R(\lambda)<\sigma_{2}$, the operators $\Lambda^{* *}$ and $P(\lambda)$ being those we have defined in introduction.

The concurrence-function $f(x)$ is now subject to the following

Assumption I. Let $f(x)$ be at least n-time differentiable, and let

$$
\int_{0}^{\infty}\left|f^{(k)}(x)\right|^{p}(1+x) e^{-\beta x} d x<\infty . \quad\left(\begin{array}{l}
p=1,2 . \\
k=0,1,2, \ldots, n
\end{array}\right)
$$

and

$$
\int_{-\infty}^{0}\left|f^{k)}(x)\right|^{p}(1+|x|) e^{-\alpha x} d x<\infty,\left(\begin{array}{l}
p=1,2 . \\
k=0,1,2 \ldots, n
\end{array}\right)
$$

where we assume $\alpha<0<\beta$.

Assumption II. For the same number $\beta$ and $\alpha$ as in (15.141) and (15.142), let

$$
f^{(k)}(x)=o\left(e^{\beta x}\right), \quad(k=0,1,2, \ldots, n)
$$

and

$$
f^{(k)}(-x)=o\left(e^{-a x}\right), \quad(k=0,1,2, \ldots, n) .
$$

as $x \rightarrow \infty$.

In addition to the assumptions with respect to the contour $\mathfrak{E}_{A}$ in the previous cases, we now assume that $\mathfrak{C}_{A}^{\prime(i)}$ coincides with $\mathfrak{S}_{\alpha+i A}^{\alpha-i A}$. Thus, in the decomposition of the integral (15.13) into four parts $\mathrm{S}_{A}^{* *}\left(x, x_{0} ; f\right)$ which correspond to $\mathfrak{S}_{A}^{(i)}(i=1,2,3,4)$ respectively, the last assumption enables us, in view of the assumptions (15.142) and (15.152), to treat the integral $\mathrm{S}_{4}^{* *}\left(x, x_{0} ; f\right)$ similarly as we have done with $\mathrm{S}_{4}\left(x, x_{0} ; f\right)$ in the previous case.

Under this modification, the similar transformation as to the previous cases will yields us readily

(15.16) $\mathrm{T}_{.1}^{* *}\left(x, x_{0} ; f\right)$

$$
\begin{aligned}
= & \frac{1}{2 \pi} \int_{-A}^{A} \frac{e^{(\beta+i t, x}}{P(\beta+i t)}\left\{\int_{x_{0}}^{\infty} e^{-(\beta+i t) \eta} f(\eta) d \eta\right\} d t \\
& +\frac{1}{2 \pi} \int_{A}^{-A} \frac{e^{(\alpha+i t) x}}{P(\alpha+i t)}\left\{\int_{x_{0}}^{-\infty} e^{-(\alpha+i t) \eta} f(\eta) d \eta\right\} d t \\
& -\frac{1}{2 \pi} \int_{-A}^{A} \frac{e^{(\beta+i t) x}}{P(\beta+i t) G^{* * * *}(\beta+i t)} \sum_{h=0}^{n} \Lambda_{0^{*}}^{* * k}\left\{e^{(\beta+i t) \xi} \int_{x_{0}+\xi}^{\infty} e^{-(\beta+i t) \eta} f^{(k)}(\eta) d \eta\right\} d t \\
& -\frac{1}{2 \pi} \int_{-1}^{-A} \frac{e^{(\alpha+i t) x}}{P(\alpha+i t) G^{* * *}(\alpha+i t)} \sum_{i=0}^{n} \Lambda_{0^{*}}^{* * k}\left\{e^{(\alpha+i t) \xi} \int_{x_{0}+\xi}^{\infty} e^{-(\alpha+i t) \eta} f^{(k)}(\eta) d \eta\right\} d t
\end{aligned}
$$




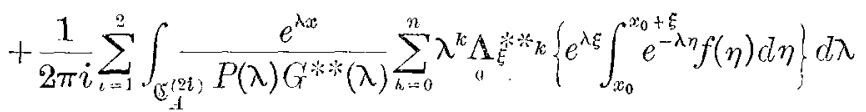

$$
\begin{aligned}
& +\frac{1}{2 \pi i} \sum_{i=1}^{2} \int_{\mathbb{C}_{\mathcal{A}}^{(2)}}^{A} \frac{e^{\lambda x}}{P(\lambda) G^{* *}(\lambda)} \sum_{k=0}^{n} \lambda^{k} \sum_{i=0}^{k-1} \frac{\Lambda_{n}^{* * *}\left\{f^{v)}\left(x_{0}+\xi\right)\right\}}{\lambda^{\nu+1}} d \lambda \text {. }
\end{aligned}
$$

\section{§16. Preliminary Considerations.}

In the sequel we will study to solve the functional equations related to $\Gamma^{*} f(x)$ and $\Gamma^{* *} f(x)$ respectively. To begin with, we introduce

Definion VIII. Under the notation $\left(S^{* \sigma}\right)$ or $\left(S^{* *}{ }_{\sigma_{1}}^{*}\right)$ we understand the function equation

$$
\Gamma_{0}^{*}\{f(x+\xi)\}=0, \quad(0 \leqq x<\infty),
$$

or

$$
\Gamma_{\xi}^{* *}\{f(x+\xi)\}=0, \quad(-\infty<x<\infty)
$$

respectively, where the linear translatable operators and the solutions $\left({ }^{5 !}\right)$ $f(x)$ satisfy the assumptions which we have made in the previous paragraph.

To solve these functional equations, we will estimate the integrals of the right-hand sides of (15.12) and (15.16). For this purpose we prepare ourselves with the following lemmas.

Lemma XVI. I. Let $f(x)$ be a solution of the functional equation $\left(S^{* \sigma}\right)$ or $\left(S_{* * \sigma_{2}}^{* *}\right)$. Let $\mathfrak{S}_{A}$ be a contour such as we have defined in $\$ 15$.

Then we have, for any non-negative numbers $x_{0}$ and $x_{1}$,

$$
\mathrm{T}_{.}^{*}\left(x, x_{0} ; f\right)=\mathrm{T}_{.}^{*}\left(x, x_{1} ; f\right) \text {, }
$$

and, for $R(\lambda) \leqq \beta$,

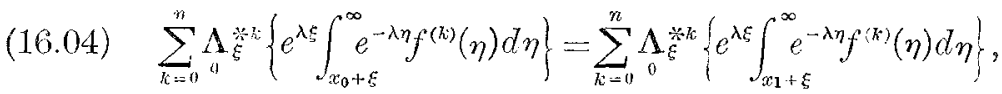
or, for any real numbers $x_{0}$ and $x_{1}$,

$$
\mathrm{T}_{A}^{* *}\left(x, x_{0} ; f\right)=\mathrm{T}_{i}^{* *}\left(x, x_{1} ; f\right) .
$$

(16.06) $\sum_{k=11}^{n} \boldsymbol{\Lambda}_{\xi}^{* * \hbar k}\left\{e^{\lambda \xi} \int_{x_{0}+\xi}^{+\infty} e^{-\lambda \eta} f^{(k)}(\eta) d \eta\right\}=\sum_{k=0}^{n} \Lambda_{\eta}^{* * *}\left\{e^{\lambda \xi} \int_{x_{1}+\xi}^{+\infty} e^{-\lambda \eta} f^{(k)}(\eta) d \eta\right\}$,

and, for $R(\lambda) \geqq \alpha$,

$$
(R(\lambda) \leqq \beta)
$$

$$
\text { (16.07) } \sum_{k=11}^{n} \Lambda_{\xi}^{*} * \ldots k\left\{e^{\lambda \xi} \int_{x_{0}+\xi}^{-\infty} e^{-\lambda \eta} f^{(k)}(\eta) d \eta\right\}=\sum_{k=0}^{n} \Lambda_{0}^{*} * k\left\{e^{\lambda \xi} \int_{x_{1}+\xi}^{-\infty} e^{-\lambda \eta} f^{(k)}(\eta) d \eta\right\}
$$

(54) For the object of solving (16.01) or (16.02), we shall make further assumptions on the behaviours of $f(x)$ at infinity. In the sequel of this chapter we assume that the interchanges of the order of the operator's $\Lambda^{*}$ (and $\Lambda^{* *}$ ) and the integration of functions which are $L_{\text {-integrable }}$ in any finite interval. are permitted. 
respectively, provided that $P(\lambda)$ is regular and has no zero-points in the half-plane $R(\lambda)<\sigma$ or in the strip $\sigma_{1}<R(\lambda)<\sigma_{\text {2 }}$ respectively.

To prove (16.03) and (16.05), we have only to remind the proof of Theorem VI. I, considering that $P(\lambda)$ is regular and has no zero-point in the domain enclosed by $\mathbb{C}_{\mathcal{A}}$ owing to the assumption.

To prove (16.04), we consider the difference

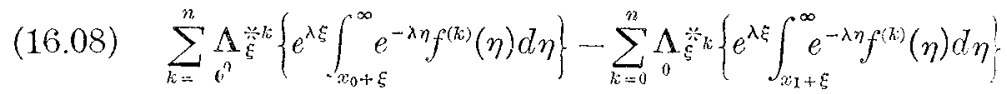

$$
\begin{aligned}
& =d\left(x_{0}, x_{1}\right), \quad s a y \text {. }
\end{aligned}
$$

changing the order of $\mathbf{\Lambda}$ and the integration over the finite interval, which is permitted by our assumption in this paragraph, (See footenote 54),

$$
\begin{aligned}
& d\left(x_{0}, x_{1}\right)=\sum_{k=0}^{n} \Lambda_{0}^{*} \xi_{\xi}^{*}\left\{e^{\lambda \xi} \int_{x_{0}+\xi}^{x_{1}+\xi} e^{-\lambda \eta} f^{(k)}(\eta) d \eta\right\} \\
& =\int_{x_{0}}^{x_{1}} e^{-\lambda \zeta} \sum_{k=0}^{n} \Lambda_{0}^{* \xi^{k}}\left\{f^{\left(x_{j}\right.}(\xi+\zeta)\right\} d \zeta \text {. }
\end{aligned}
$$

Thus, since $f(x)$ is a solution of $\left(S^{* \sigma}\right)$, we obtain

$$
d\left(x_{0}, x_{1}\right)=0
$$

which we were to prove.

The relations (16.06) and (16.07) may be proved quite similary.

Lemma XVI. II. Let $f(x) e^{-\beta x}$ belong to $L^{2}\left(x_{0}, \infty\right)$. Let $P(\lambda)$ be a polynomial in $\lambda$ not ranishing on the line $R(\lambda)=\beta$.

Then

$$
\underset{\lambda \rightarrow \infty}{\lim } \frac{1}{2 \pi} \int_{-\alpha}^{1} \frac{e^{(\beta+i t) x}}{P(\beta+i t)}\left(\int_{x_{0}}^{\infty} e^{-\langle\beta+i t) \eta} f(\eta) d \eta\right) d t
$$

exists, and if we denote this limit in the mean by $\varphi(x)$, then we have, in symbolic notation,

$$
P\left(D_{x}\right) \varphi(x)=f(x), \quad\left(x_{0}<x<\infty\right) .
$$

Since the existonce of (16.09) follows readily from the assumptions, we have only to prove (16.12). Now by formal differentiations, we have

$$
\lim _{A \rightarrow \infty} \frac{1}{2 \pi} \int_{-1}^{A} \frac{(\beta+i t)^{\prime \prime} e^{(\beta+i t) x}}{P(\beta+i t)}\left(\int_{x_{0}}^{\infty} e^{-(\beta+i t) \eta} f(\eta) d \eta\right) d \dot{t}=\varphi^{(\nu)}(x)
$$

where $\nu$ is non-negative integer not greater than the degree of the poì. nomial $P(\lambda)$.

Therefore we may obtain (16.12) formally. But these formal calculations may be justified by the fact that $f(x) e^{-\beta x}$ belongs to $L^{2}(x,+\infty)$ and that 
(16.14)

$$
\begin{aligned}
e^{\lambda . x} \int_{0}^{x} e^{-\lambda \xi} & \left\{\lim _{-1 \rightarrow \infty} \cdot \frac{1}{2 \pi} \int_{-\Lambda}^{-1} e^{(\beta+i t) \eta}\left(\int_{x 0}^{\infty} e^{-(\beta+i t) \eta} f(\eta) d \eta\right) d t\right\} d \xi \\
= & \frac{e^{\lambda x}}{2 \pi} \int_{-\infty}^{\infty} \frac{e^{(\beta+i t-\lambda) x}}{\beta+i t-\lambda}\left(\int_{x_{0}}^{\infty} e^{-(\beta+i t) \eta} f(\eta) d \eta\right) d t \\
& -\frac{e^{\lambda x}}{2 \pi} \int_{-\infty}^{\infty} \frac{1}{\beta+i t-\lambda}\left(\int_{x_{0}}^{\infty} e^{-(\beta+i t) \eta} f(\eta) d \eta\right) d t
\end{aligned}
$$

where $R(\lambda) \neq \beta$.

Now decomposing the differential operator $P(D)$ into

$$
P(D)=c \prod_{v=0}^{k}\left(D-\lambda_{v}\right)
$$

we have, by our assumption, $R\left(\lambda_{v}\right) \neq \beta(\nu=1,2, \ldots, k)(k>2)$. Therefore we are able to use (16.14) repeatedly, and get our theorem.

Here we will adopt the following

Definition IX. I. If there corresponds to a linear translatable operator $\Lambda^{*}\left(\right.$ or $\left.\Lambda^{* *}\right) \bar{\Lambda}^{*}$ (or $\left.\bar{\Lambda}^{* *}\right)$ such that

1. If $f(t)$ belongs to the domain of $\Lambda^{*}$ (or $\left.\Lambda^{* * *}\right)$, then it belongs to the domain of $\overline{\mathbf{\Lambda}}^{*}$ (or $\overline{\mathbf{\Lambda}}^{*}$ ) also, and we have

$$
\left(\begin{array}{lc}
(16.16) & \left|\underset{0}{\Lambda_{\xi}^{*}}\{f(x+\xi)\}\right| \leqq \bar{\Lambda}_{0}^{*}\{|f(x+\xi)|\} \\
o r & \left.\left|\underset{0}{\Lambda_{\xi}^{*} *}\{f(x+\xi)\}\right| \leqq \bar{\Lambda}_{0}^{*} * f|f(x+\xi)|\right\}
\end{array}\right) .
$$

2. If $f(t)$ belongs to the domain of $\mathbf{\Lambda}^{*}$ (or $\mathbf{A}^{* * *}$ ), and is non-negative in $(0, \infty)$ (or in $(-\infty, \infty))$ then, respectively,

$$
\left(\begin{array}{ll}
(16.18) & \bar{\Lambda}_{0}^{*}\{f(x+\xi)\} \geqq 0, \\
o r & \bar{\Lambda}_{0}^{*} *\{f(x+\xi)\} \geqq 0
\end{array}\right.
$$

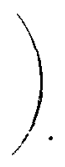

3. The generating function of the operator $\Lambda^{*}$ (or $\bar{\Lambda}^{* *}$ ), which we denote by $G^{*}(\lambda)$ (or $G^{* *}(\lambda)$ ), is bounded in any strip defined by $0 \leqq R(\lambda)$ $\leqq \alpha-\varepsilon$ (or $\left.\alpha_{1}+\varepsilon \leqq R(\lambda) \leqq \alpha_{2}-\varepsilon\right)$, where $\varepsilon$ is any given positive number, then the linear translatable operator $\Lambda^{*}$ (or $\left.\Lambda^{* *}\right)$ is called to be capable of normalisation. We denote this property by $\left(N^{* \sigma}\right)$ (or $\left.\left(N_{\sigma_{1}}^{* \% \sigma_{1}}\right)\right)$.

In virtue of the properties $\left(N^{* r}\right)$ and $\left(N^{* * \sigma_{2}}\right)$, we get the following

Lemma XVI.III. In addition to the assumptions imposed upon the linear translatable operator $\Gamma^{*} f$ and $\Gamma^{* *} f$ in the paragraph, let us assume

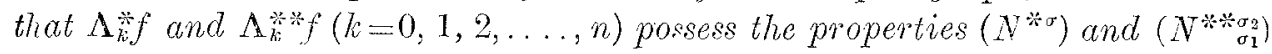
respectively. 
Then we have the estimations to the following effects:

1. Let $f(x)$ be L-integrable in any finite range of the positive $x$-axis, and let it be

(16.20)

$$
f(x)=O\left(e^{\beta i}\right), \quad(\text { as } x \rightarrow \infty),
$$

where $\beta$ is subject to $\beta<\sigma$.

Then, for any $\lambda$ which is subject to $R(\lambda) \leqq \beta-\varepsilon$, $\varepsilon$ being any positive number, we have

$$
\sum_{i=0}^{n} \Lambda_{0}^{*}\left\{e^{k \xi} \int_{0}^{\xi} e^{-\lambda \eta} f(\eta) d \eta\right\}=O\left(\frac{1}{\varepsilon}\right)
$$

2. Let $f(x)$ be L-integrable in any finite range of the $x$-axis, and let it be

$$
f(x)=O\left(e^{\beta \cdot x}\right), \quad(\text { as } x \rightarrow \infty)
$$

and

$$
f(x)=O\left(e^{\alpha x}\right), \quad(\text { as } x \rightarrow-\infty)
$$

where $\alpha$ and $\beta$ are subject to $\sigma_{1}<\alpha<\beta<\sigma_{\text {s. }}$

Then, for any number $\lambda$ such that $R(\lambda)$ belongs to the interval $(\operatorname{Min}(\alpha, \beta)+\varepsilon, \operatorname{Max}(\alpha, \beta)-\varepsilon), \varepsilon$ being any given positive number, we have

$$
\sum_{k=0}^{n} \mathbf{A}_{0}^{* *}\left\{e^{\lambda \xi} \int_{0}^{\xi} e^{-\lambda \eta} f(\eta) d \eta\right\}=O\left(\frac{1}{\varepsilon}\right)
$$

This may be easily seen; for example, to get (16.21), we have only to obtain the estimation to the effect: by the assumption (16.20), we have, for $\lambda$ now in consideration,

$$
e^{\lambda \xi} \int_{0}^{\xi} e^{-\lambda \eta} f(\eta) d \eta \leqq K\left\{\frac{e^{\beta \xi}}{\varepsilon}+\frac{e^{(\beta-\varepsilon) \xi}}{\varepsilon}\right\}
$$

where $K$ is a positive number independent of $\xi$. Therefore it follows that

$$
\begin{aligned}
& \mid \sum_{k=0}^{n} \Lambda_{\xi}^{* k}\left\{e^{\lambda \varepsilon} \int_{0}^{\xi} e^{-\lambda \eta} f(\eta) d \eta\right\} \\
& \leqq \sum_{k=0}^{n} \bar{\Delta}_{\bar{n}} \bar{k}_{\xi}\left\{e^{\lambda \xi} \int_{0}^{\xi} e^{-\lambda \eta} f(\eta) d \eta\right\} \\
& \leqq \frac{2 K}{\varepsilon} \sum_{k=0}^{n} \bar{\Lambda}_{0}^{k k}\left\{e^{\beta \xi}\right\}=O\left(\frac{1}{\varepsilon}\right) \text {, }
\end{aligned}
$$

where we have used the properties $1^{\circ}, 2^{\circ}, 3^{\circ}$ of $\bar{\Lambda}^{* k}$.

It will be useless to show an example of the linear translatable operator $\Lambda^{*} f$ with the property $\left(N^{* \alpha}\right)$ : let us consider the linear translatable operator

$$
\Lambda^{*} f(x)=\int_{0}^{\infty} f(x+t) K(t) d t
$$


and let it be assumed that, for a certain $\sigma>0$,

$$
\int_{0}^{\infty} e^{\sigma t}|K(t)| d t<\infty
$$

Then the operator defined by

$$
\bar{\Lambda}^{*} f(x)=\int_{0}^{\infty}|f(x+t)||K(t)| d t
$$

satisfies the conditions imposed in Def. IX.I, and therefore $\mathbf{A}^{*} f(x)$ has the property $\left(N^{* \sigma}\right)$.

Lastly, in order to estimate the cxpressions of the left-hand sides of (16.04), (16.06) and (16.07), which, for the sake of simplicity, we will denote hereafter by $N^{+}\left(\lambda, x_{*} ; \Gamma^{*}: f\right), N^{+}\left(\lambda, x_{0} ; \Gamma^{* *}: f\right)$ and $N^{-}\left(\lambda, x_{0} ; \Gamma^{* *}: f\right)$ respectively, we will now introduce the following

Definition IX. II. Let there be a sequence of the linear translatable differential operators $\Gamma^{(m)} f(x)$ with the following properties:

$$
\Gamma^{(m)} f(x) \equiv \sum_{k=0}^{n} \Lambda^{(m,}, f^{(i)}\left\{f^{(k)}(x+\xi)\right\}, \quad(m=1,2,3, \ldots)
$$

where the generating functions of $\Lambda^{(m, k)}$ are all integral functions.

2. For any given positive number $s$, we are able to choose a number $M(\varepsilon)$ such that we have, for $m>M(\varepsilon)$,

$$
\left|N^{+}\left(\lambda, x_{0} ; \Gamma^{*}: f\right)-N^{*}\left(\lambda, x_{0} ; \Gamma^{(n)}: f\right)\right|<\varepsilon, \quad(R(\lambda)=\beta)
$$

uniformly concerning $x_{1}$ in $(0, \infty)$, or

$$
\left|N^{+}\left(\lambda, x_{0} ; \Gamma^{* *}: f\right)-N^{+}\left(\lambda, x_{0} ; \Gamma^{(m)}: f\right)\right|<\varepsilon, \quad(R(\lambda)=\beta)
$$

uniformly concerning $x_{0}$ in $(0, \infty)$, and

$$
\left|N^{-}\left(\lambda, x_{1} ; \Gamma^{\circ *}: f\right)-N^{-}\left(\lambda, x_{0} ; \Gamma^{(m)}: f\right)\right|<\varepsilon, \quad(R(\lambda)=\alpha)
$$

uniformly concerning $x_{0}$ in $(-\infty, 0)$.

Then we say that the linear translatable differential operator $\Gamma^{*} f(x)$ and $\Gamma^{* *} f(x)$ have the property $\left(U^{* \beta}\right)$ and the property $\left(U^{* * \beta}\right)$ respectively.

These properties leads us to the following:

Lemma XVI. IV. In addition to the assumptions of Lemma XVI.I., let us assume furthermore that the linear translatable differential operator. $\Gamma^{*} f(x)$ and $\Gamma^{* *} f(x)$ possess the property $\left(U^{* \beta}\right)$ and (U⿳亠丷⿵冂丶

Then we have

$$
N^{++}\left(\beta+i t, x_{0} ; \Gamma^{*}: f\right)=0,
$$

or

(16.321)

$$
N^{+}\left(\beta+i t, x_{0} ; \Gamma^{* *}: f\right)=0
$$

and

$$
N^{-}\left(\alpha+i t, x_{v} ; \Gamma^{* *}: f\right)=0
$$


respectively.

To see (16.31) let us notice that, in virtue of Lemma XVI.I. and the property $\left(U^{* 3}\right)$, we may write

(16.38)

$$
\begin{aligned}
N^{*} & \left(\beta+i t, x_{0} ; \Gamma^{*}: f\right) \\
& =\lim _{x_{0} \rightarrow \infty} N^{+}\left(\beta+i t, x_{0} ; \Gamma^{*}: f\right) \\
& =\lim _{x_{0} \rightarrow \infty}\left[\lim _{m \rightarrow \infty} N^{+}\left(\beta+i t, x_{i} ; \Gamma^{(m)}: f\right)\right] \\
& =\lim _{m \rightarrow \infty}\left[\lim _{x_{n} \rightarrow \infty} N^{+}\left(\beta+i t, x_{i} ; \Gamma^{(m)}: f\right)\right] .
\end{aligned}
$$

Here remembering the considerations in (8.05)-(8.07), we get

$$
\lim _{m \rightarrow \infty} N^{+}\left(\beta+i t, x_{0} ; \Gamma^{(m)}: f\right)=0 .
$$

Combining (16.33) and (16.34) we obtain (16.31).

The relations (16.321) and (16.332) may be proved quite similarly.

Since the properties $\left(U^{* 8}\right)$ and $\left(U_{a}^{* * 3}\right)$ seem somewhat abstract and artificial, it may be worth while to give the following

Corollary XVI.V. Let the linear translatable differential operators now in our consideration be given by

$$
\Gamma^{*} f(x) \equiv \sum_{k=0}^{n} \int_{0}^{\infty} f^{(k)}(x+t) \varphi_{k}(t) d t, \quad\left(\int_{1}^{\infty} e^{\sigma t}\left|\varphi_{k}(t)\right| d t<\infty\right),
$$

and

$$
\Gamma^{* *} f(x)=\sum_{k=1}^{n} \int_{-\infty}^{\infty} f^{\prime(k)}(x+t) \varphi_{k}(t) d t, \quad\left(\int_{10}^{ \pm \infty} e^{\sigma_{i} t}\left|\varphi_{k}(t)\right| d t<\infty\right) .
$$

Let $f(x)$ be a solution of the functional equation $\left(S^{* \sigma}\right)$ or $\left(S^{* * \sigma_{2}}\right)$ associated to one of these operators, and in addition to the assumptions of the concurrence-functions in $\$ 15$, let us assume furthermore that

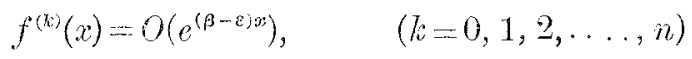

as $x \rightarrow \infty$, or

(16.881)

$$
f^{(k)}(x)=O\left(e^{(\beta-\varepsilon) x}\right), \quad(k=0,1,2, \ldots, n)
$$

as $x \rightarrow \infty$, and

$$
\text { (16.382) } \quad f^{(k}(x)=O\left(e^{(\alpha+\varepsilon) x}\right), \quad(k=0,1,2, \ldots, n)
$$

as $x \rightarrow-\infty$, respectively, $\varepsilon$ being a positive number such that

$$
\sigma_{1}<\alpha+\varepsilon<\beta-\varepsilon<\sigma_{3} .
$$

Then $\Gamma^{*} f(x)$ satisfies the condition $\left(U^{* 3}\right)$ with respect to the sequence of the operators

$$
\Gamma^{(m)} f(x) \equiv \sum_{k=1}^{n} \int_{0}^{i n} f^{(k)}(x+t) \varphi_{l}(t) d t, \quad(m=1,2, \ldots, n, \ldots)
$$

and $\Gamma^{* *} f(x)$ satigies the condition $\left(U_{a}^{* * 3}\right)$ with respect to the sequence of 
the operators

$$
\Gamma^{(m)} f(x) \equiv \sum_{k=1}^{n} \int_{-m}^{m} f^{(k)}(x+t) \varphi_{k}(t) d t, \quad(m=1,2, \ldots, n, \ldots) .
$$

Proof. We will prove these facts merely for $\Gamma^{* *} f(x)$. The romaining case will then be easily seen.

To begin with, let us estimate tho integral

$$
\int_{x_{0}+\xi}^{\infty} e^{-\langle\beta+i \ell \eta \eta} f^{\prime k)}(\eta) d \eta=J^{k}\left(x_{0}+\xi\right)
$$

say.

First let $x_{0}+\xi \geqq 0$. Then we have

$$
\left|J^{k}\left(x_{0}+\xi\right)\right| \leqq M_{k} \int_{0}^{\infty} e^{-\beta \eta} \cdot e^{(\beta-\varepsilon) \eta} d \eta=-\frac{M_{k}}{\varepsilon} .
$$

Next let $x_{0}+\xi<0$. Then, assuming $x_{0}>0$, we now obtain

$$
\begin{aligned}
\left|J^{k}\left(x_{0}+\xi\right)\right| & \leqq \\
\leqq & e^{-(\beta+i t) x_{0}} \int_{-x_{0}}^{\infty} e^{-(\beta+i t) \zeta} f^{(k)}\left(x_{0}+\zeta\right) d \zeta \mid \\
& +\mid e^{-(\beta+i t) x_{0}} \int_{\xi}^{-x_{0}} e^{-(\beta+i t) \zeta} f^{(k)}\left(x_{0}+\zeta\right) d \zeta \\
\leqq & M_{k} e^{-\beta x_{0}} \int_{-x_{0}}^{\infty} e^{-\beta \zeta} \cdot e^{(\beta-\varepsilon)\left(x_{0}+\zeta\right)} d \zeta+L_{k} e^{-\beta x_{0}} \int_{\xi}^{-x_{0}} e^{-\beta \zeta} \cdot e^{(\alpha+\varepsilon)\left(x_{0}+\zeta\right)} d \zeta \\
\leqq & \frac{M_{k}}{\varepsilon}+\frac{L_{k}}{|\alpha+\varepsilon-\beta|}\left[1+e^{(\alpha+\varepsilon-\beta)\left(x_{0}+\xi\right)}\right] .
\end{aligned}
$$

Thus we get

$(16.45)$

$$
\begin{aligned}
N^{++}\left(\beta+i t, x_{0} ;\right. & \left.\Gamma^{* *}: f\right)-N^{+}\left(\beta+i t, x_{i} ; \Gamma^{(m)}: f\right) \\
= & \sum_{k=0}^{n} \int_{-\infty}^{-m} e^{(\beta+i t) \xi}\left(\int_{x_{0}+\xi}^{\infty} e^{-(\beta+i t) \eta} f^{(k)}(\eta) d \eta\right) \varphi_{k}(\xi) d \xi \\
& +\sum_{k=0}^{n} \int_{m}^{\infty} e^{(\beta+i t) \xi}\left(\int_{x_{0}+\xi}^{\infty} e^{-(\beta+i t) \eta} f^{(k)}(\eta) d \eta\right) \varphi_{k}(\xi) d \xi \\
= & \sum_{k=0}^{m}\left(\int_{-\infty}^{-m}+\int_{-m}^{\infty}\right) e^{(\beta+i t) \xi} J\left(x_{0}+\xi\right) \varphi_{k}(\xi) d \xi \\
= & \sum_{k=0}^{n} O\left(\left(\int_{-\infty}^{-m}+\int_{m}^{\infty}\right) e^{\beta \xi}\left|\varphi_{k}(\xi)\right| d \xi\right) \\
& +\sum_{k=0}^{n} O\left(\left(\int_{-\infty}^{-m}+\int_{m}^{\infty}\right) e^{(\alpha+\varepsilon ; \xi}\left|\varphi_{k}(\xi)\right| d \xi\right) \mid e^{(\alpha+\varepsilon-\beta) x_{0} \mid}
\end{aligned}
$$

Since $\alpha+\varepsilon-\beta<0, x_{0}>0$, the last relation shows us that for any given positive $\delta$ we are able to choose a number $M(\delta)$ such that we have for any $m \geqq M(\delta)$, 


$$
\left|N^{+}\left(\beta+i t, x_{0} ; \Gamma^{* *}: f\right)-N^{+}\left(\beta+i t, x_{0} ; x_{0} ; \Gamma^{(m)}: f\right)\right|<\delta,
$$

uniformly concerning $x_{0}$ in $(0, \infty)$, which we were to prove.

The remaining may be proved similarly.

\section{$\S 17$. General Linear Translatable Functional Equations $(\boldsymbol{S} * \sigma)$ and $\left(\boldsymbol{S} * \underset{\sigma_{1}}{*}\right)$.}

We are now in position to give the expansion-theorems for the solutions of the general linear translatable functional equation $\left(S^{\% \sigma}\right)$ or $\left(S_{\sigma_{2}}^{* \sigma_{2}}\right)$. We will begin with

Theorem XVII. I. Let $f(x)$ be a solution of the functional equation $\left(S^{* \sigma}\right)$, that is, we have defined,

(17.01) $\quad \Gamma^{*} f(x)=0, \quad(0 \leqq x<\infty)$

where the generating functions $G_{*}^{*}(\lambda)$ of $\Lambda^{*}$ are regular in the half plane $R(\lambda)<\sigma,(k=0,1,2, \ldots, n)$, and let, in addition to Assumption $I$,

(17.02)

$$
f^{(k)}(x)=O\left(e^{(\beta-\varepsilon) x}\right), \quad(k=0,1,2, \ldots, n)
$$

as $x \rightarrow \infty$, where we assume

$$
\beta-\varepsilon<\beta<\sigma .
$$

Further let the operators $\Gamma^{*} f(x)$ possess the properties $\left(N^{*}\right)$ and $\left(U^{* \beta}\right)$.

If there is a sequence of contours $\left\{\mathfrak{C}_{s_{m}}\right\}$ such as we have defined in $\$ 15.1$ with the following additional properties:

(i) There is a positive number $S$ independent of $n$ such that

$$
\operatorname{Min}_{\lambda \text { on } \mathfrak{C}_{i m}^{(i)}}\left|G_{n}(\lambda)\right|>S>0, \quad(i=2,3,4)
$$

(ii) The distances between $\mathfrak{S}_{A n}^{(i)}(i=2,3,4)$ and the origin tend to infinity as $m \rightarrow \infty$, then the contour-integral $\mathrm{T}_{A_{m}}^{*}(x, 0 ; f)$ defined for this sequence of the contours $\left\{\mathfrak{S}_{\boldsymbol{A}_{m}}\right\}$ and $P(\lambda)=c \prod_{i=1}^{k}\left(\lambda-\lambda_{i}\right),(R(\lambda)>\sigma)(i=1,2, \ldots, k),(k \geqq 2)$ as in $\$ 15$ tends in the mean to a function $\varphi(x)$, and we have,

$$
P\left(D_{x}\right) \varphi(x)=f(x), \quad(0<x<\infty) .
$$

To prove this, we recall in mind the formulue (15.12), and we will estimate the right-hand side of it in the following ways:

$1^{\circ}$. As $x_{0}=0$, the second term does not exist.

$2^{\circ}$. In virtue of the present assumptions, the hypothesis to Lemma XVI. IV is satisfied, and therefore the third term is equal to zero for any $n$.

$3^{\circ}$. By the assumption (17.02) we may appeal to Lemma XVI. III, which gives us

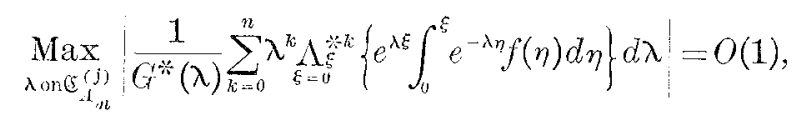


and

$$
\operatorname{Max}_{\lambda \text { on } \mathfrak{E}_{d_{m}}^{(j)}}\left|\frac{1}{G^{*}(\lambda)} \sum_{k=1}^{n} \lambda^{k} \sum_{v=1}^{k-1} \frac{\Lambda_{\xi=0}^{* \xi^{k}}\left\{f^{(v)}\left(x_{0}+\xi\right)\right\}}{\lambda^{v+1}}\right|=O(1),
$$

as $m \rightarrow \infty$, for $j=2,3,4$.

Therefore, since, the distances between $\left\{\mathfrak{C}_{i}^{(i)}\right\}(j=2,3,4)$ and the origin tend to infinity as $m \rightarrow \infty$, the fifth and the sixth integrals tend to zero as $m \rightarrow \infty$.

$4^{\circ}$. Since $P(\lambda)$ is regular and has no zero-points in the domain enclosed by $\mathfrak{S}_{-i}$, the fourth term is equal to zero.

After these considerations we see that only the first term of the righthand side of (15.12) makes contribution, which, in virtue of Lemma XVI. II, leads us to the conclusion (17.05), as we were to prove.

Theorem XVII. II. Let $f(x)$ be a solution of the functional equation $\left(S^{*}{ }_{\sigma_{1}}^{*}\right)$, that $i s$, as we have defined,

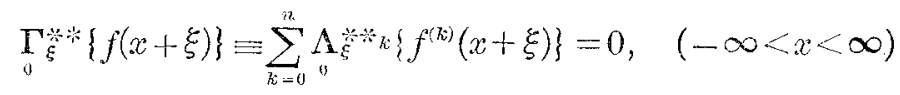

where the generating functions $G_{k}^{*} *(\lambda)$ of $\Lambda_{*}^{* *}$ kare regular in the strip $\sigma_{1}<R(\lambda)<\sigma_{2}(k=0,1,2, \ldots, n)$, and let, in addition to Assumption $I$,

$$
f(x)=O\left(e^{(\beta-\varepsilon) x)}, \quad(k=0,1,2, \ldots, n)\right.
$$

as $x \rightarrow \infty$, and

$$
f(x)=O\left(e^{(\alpha+\varepsilon) x}\right), \quad(k=0,1,2, \ldots, n)
$$

as $x \rightarrow-\infty$, where we assume

$$
\sigma_{1}<\alpha<\alpha+\varepsilon<\beta-\varepsilon<\beta<\sigma_{2} .
$$
$\left.U^{* * \%}\right)$.

Further let the operators $\Gamma^{* *} f(x)$ possess the properties $\left(N_{a * \beta}^{* *}\right)$ and

If there is a sequence of contours $\left\{\mathfrak{S}_{x_{n}}\right\}$ such as we have difined in $\$ 15.2$ with the following additional properties:

(i) There is a positive number $S$ independent of $n$ such that

$$
\operatorname{Min}_{\lambda \circ \mathbb{C}_{i m}^{(i)}}\left|G_{n}(\lambda)\right|>S>0, \quad(i=2,4)
$$

(ii) The distances between $\mathfrak{C}_{A_{m}}^{(i)}(i=2,4)$ and the origin tend to infininty as $m \rightarrow \infty$.

Then the contour-integral $\mathrm{T}_{m} *{ }_{m}^{*}(x, 0: f)$ defined for this sequence of the contours $\left\{\mathbb{E}_{-i_{m}}\right\}$ as in $\$ 15.9$. tends in the mean to a function $\varphi(x)$ such that we have

$$
P\left(D_{x}\right) \varphi(x)=f(x),
$$

where we put 


$$
P(D)=c \prod_{i=1}^{k}\left(D-\lambda_{i}\right), \quad\left(\left|R\left(\lambda_{i}\right)\right|>\left|\sigma_{2}\right|,\left|\sigma_{1}\right|, \quad k \geqq 2\right) .
$$

To prove this, we remind the formula (15.16). We may estimate the third and the following terms of its right-hand side similarly as those of (15.12).

Now by our assumptions

$$
\lim _{m \rightarrow \infty} \frac{1}{2 \pi} \int_{-A_{m}}^{A_{m}} \frac{e^{(\beta+i t) x}}{P(\beta+i t)}\left(\int_{0}^{\infty} e^{-(\beta+i t) \eta} f(\eta) d \eta\right) d t
$$

and

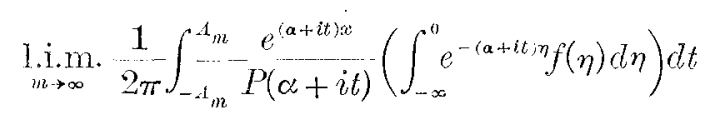

will exist. If we denote by $\varphi_{1}(x)$ and $\varphi_{2}(x)$ these limits in the mean respectively, then we have, by Lemma XVI. II.,

and

$$
\begin{aligned}
& P\left(D_{x}\right) \varphi_{1}(x)=f(x), \quad(x>0), \\
& P\left(D_{x}\right) \varphi_{1}(x)=0, \quad(x<\hat{0}),
\end{aligned}
$$

Therefore we have

$$
\begin{aligned}
& P\left(D_{x}\right) \varphi_{2}(x)=f(x), \quad(x<0), \\
& P\left(D_{x}\right)_{2}(x)=0, \quad(x>0) \text {. }
\end{aligned}
$$

and

$$
\varphi(x)=\varphi_{1}(x)+\varphi_{2}(x)
$$

In combination of the well-known convergence-theorems of Fourierintegral, these two theorems will yield us

Theorem XVII. III. If we assume, in addition to the lypothesis of Theorem XVII. I. or Theorem XVIT. I., that $f(x)$ is of bound variation in any finite range, which is always satisfied for the case $n \geqq 1$, then, in the conclusion of Theorem XVII. I. or Theorem XVII. II., we may replace "the limit in the mean" by the ordinary limit; that is, we have

$$
\lim _{m \rightarrow \infty} \mathrm{T}_{-i_{m}}^{*}(x, 0 ; f)=\varphi(x) \text {, }
$$

$O i^{2}$

$$
\lim _{m \rightarrow \infty} \mathrm{T}_{m}^{* *}(x, 0 ; f)=\varphi(x)
$$

respectively,

\section{§18. Applications of Convergence-Theorems.}

Bochner $\left({ }^{(j i}\right)$ proved the theorcm to the following effect: let $f(x)$ be a (5.5) Bochner [9] Satz I. (S. 439). 
solution of the difference-equrtion

$$
E f(x) \equiv \sum_{i=0}^{n} A_{i} f\left(x+\alpha_{i}\right)=0, \quad(-\infty<x<\infty)
$$

Let us. denote by $\mu_{n}$ the real parts of the zero-point $\lambda_{n}$ of the generating function

$$
G\left(\lambda_{n}\right)=0
$$

and by $M$ the closed set consisted of all such $\mu_{u}$ and their accumulationpoints.

Then we have

$$
f(x) \equiv 0
$$

or

$$
G(f) \in \mathfrak{M}
$$

In connection with this theorem, it may be not useless to mention

Theorem XVIII. I. If in addition to the hypothesis to Theorem XVII. I. or Theorem XVII.II., we assume that there are none of zero-points of the generating function in the interior of $\left\{\mathfrak{S}_{A}\right\}$, then

$$
f(x) \equiv 0
$$

In the Schürer's theory $\left({ }^{56}\right)$ of the functional equations, certain equations may be reduced to differential equations of finite order with constant coefficients, which may be recognised to be intimately connected with

Theorem XVIII. II. If, in addition to the hypothesis to Theorem XVIT. I. or Theorem XVII. II., we assime that there are finite number of zero-points of the generating function in the interior of $\left\{\mathfrak{S}_{A}\right\}$, then the functional equation $\left(S^{* \sigma}\right)$ or $\left(S^{* * \sigma_{0}}\right)$ reduces to differential equation of finite order with constant coefficients.

These two theorems may be readily proved in virtue of the theorems given in the previous paragraphs.

\section{§19. Examples (Singular Integral Equations).}

Let us consider so-called singular integral equations

$$
f(x)=\int_{0}^{\infty} f(x+t) K(t) d t
$$

and

If we assume that

$$
f(x)=\int_{-\infty}^{\infty} f(x+t) K^{-}(t) d t
$$

$\left.{ }^{56}\right)$ Schürer [1] C. L-gleichungen unendlich hoher Ordnung. 2. Alle Lösungen der $L$ gleichung unendlich hoher Ordnung im Bereiche $\left[L^{\infty}, q\right]$ sind Lösungen einer $I$-gleichung endlicher Ordnung. S. 210-211. also Satz. IV S. 214-215. 


$$
G^{\circ} \stackrel{*}{*}(\lambda)=1-\int_{0}^{\infty} e^{\lambda t} K(t) d t
$$

and

$$
G^{* * *} *(\lambda)=1-\int_{-\infty}^{\infty} e^{\lambda t} K(t) d t
$$

are regular in the half-plane $R(\lambda)<\sigma$ and in the strip $\sigma_{1}<R(\lambda)<\sigma_{2}$ respectively, then the above functional equations become the type $\left(S^{* \sigma}\right)$ and $\left(S^{*} \underset{\sigma_{1}}{* \sigma_{2}}\right)$ respectively.

Further we may easily rerify that the conditions imposed upon the operator and the generating function in Theorem XVII.I. or Theorem XVIIII. are satisfied. For example, writing $\lambda=\tau+i A$, we have

$$
\left|G^{* *}\left(\tau \pm i A_{m}\right)\right|>S>0, \quad \text { as } m \rightarrow \infty
$$

uniformly in any strip $\xi_{1} \leqq R(\lambda) \leqq \xi_{2}$ subject to $\sigma_{1}<\xi_{1}<\xi_{2}<\sigma_{2}$.

Thus we may appeal to the theorems above mentioned.

On the other hand we notice that our method is not applicable to solve the singular integral equation

$$
f(x)=\int_{0}^{\infty} f(x-t) K(t) d t .
$$

For the operator $H f(x)$ defined by

$$
H f(x) \equiv f(x)-\int_{0}^{\infty} f(x-t) K(t) d t
$$

is not permittable with translation.

\section{Chapter V. Principal Solution of Non-homogeneous Linear Translatable Functional Equation.}

\section{\$20. Solution under Integral Representation.}

Let us consider the non-homogeneous linear translatable functional equation:

$$
\text { (20.01) } \quad \Gamma f(x)=g(x), \quad(-\infty<x<\infty)
$$

where $\Gamma$ is a linear translatable functional transformation (0.24) defined in Introduction.

To solve this functional equation. we will remark that the integrals

$$
\begin{aligned}
N_{r}\left(x, x_{0} ; g\right)= & \frac{1}{2 \pi i} \int_{x_{0}}^{x_{0}+b} g(t)\left\{\int_{\mathscr{C}_{r}^{(+,)}} \frac{e^{\lambda(x-t)}}{G(\lambda)} d \lambda\right\} d t \\
& +\frac{1}{2 \pi i} \int_{x_{0}}^{x_{0}+\alpha} g(t)\left\{\int_{\tilde{E}_{r}^{\prime-)}} \frac{e^{\lambda(x-t)}}{G(\lambda)} d \lambda\right\} d t
\end{aligned}
$$

satisfies the functional equation approximately in a certain sense; that is, 
if we operate $\Gamma_{x}$ to $N_{r}\left(x, x_{0} ; g\right)$, then we have

$$
\begin{aligned}
\Gamma_{0}\left\{N_{r}\left(x+s, x_{0} ; g\right)\right\} \\
=\frac{1}{2 \pi i} \int_{x_{0}}^{x_{0}+b} g(t)\left(\int_{\mho_{r}^{(+)}} e^{\lambda(x-t)} d \lambda\right) d t \\
\quad+\frac{1}{2 \pi i} \int_{x_{0}}^{x_{0}+a t} g(t)\left(\int_{\sigma_{r}^{(-)}} e^{\lambda(x-t)} d \lambda\right) d t
\end{aligned}
$$

which gives us

$$
\lim _{i \rightarrow \infty} \Gamma_{s}\left\{N_{i}\left(x+s, x_{0} ; g\right)\right\}=g(x)
$$

provided that

$$
\lim _{r \rightarrow \infty} \frac{1}{\pi} \int_{x_{0}+a}^{x_{0}+b} g(t) \frac{\sin \rho_{r}(x-t)}{x-t} d t=g(x)
$$

Therefore we obtain

Theorem XX.I. If there is a sequence of contours $\left\{\mathfrak{E}_{i}\right\}$ such that

$$
\lim _{y \rightarrow \infty} N_{r}\left(x, x_{0} ; g\right)=N\left(c, x_{0} ; g\right) \quad(s a y)
$$

exists and $N\left(x, x_{0} ; g\right)$ is L-integrable in $x_{0}+a<x<x_{0}+b$, then

$$
\Gamma_{0}\left\{N\left(x+s, x_{0} ; g\right)\right\}=g(x),
$$

provided that $(20.05)$ is satisfied.

\section{§21. Preliminary Consideration.}

In the previous paragraph we have given the solution of the functional equation (20.01) as a limiting function of certain integrals. As a matter of practice, however, we nced the solutions which are exprossed in a form to be more easily handled with.

There are three ways to proceed for this purpose.

The first way is the employment of generalised Bernoull's polynomials. Let, for example, the origin $\lambda=0$ be a zero-point of the order $k$ of the generating function $G(\lambda)$.

Then we consider the function

$$
\frac{\lambda^{k} e^{\lambda(x+h t h-t)}}{G(\lambda)}
$$

which is regular in a certain circle $|\lambda|<\rho$. We expand this function into power series of $\lambda$ as follows:

$$
\frac{\lambda^{k} e^{\lambda(x+h-t)}}{G(\lambda)}=\sum_{s=0}^{\infty} B_{s}(h) e^{\lambda(x-t)} \lambda^{s} .
$$

Substitnting this into (20.02), we obtain 
$(21.03)$

$$
\begin{aligned}
N_{r}\left(x, x_{0} g\right) & =\frac{1}{2 \pi i} \int_{x_{0}}^{x_{0}+b} g(t)\left\{\int_{\mathbb{C}_{r}^{(+)}} \sum_{s=0}^{\infty} B_{s}(h) e^{\lambda(x-t} \lambda^{s-k} d \lambda\right\} d t \\
& +\frac{1}{2 \pi i} \int_{x_{0}}^{x_{0}+a} g(t)\left\{\int_{\mathbb{E}_{r}} \sum_{-, s=0}^{\infty} B_{s}(h) e^{\lambda(x-t)} \lambda^{s-k} d t\right\} d \lambda
\end{aligned}
$$

Due to the uniform convergence of the series of (21.02), we may change the order of the summations and the integrations in (21.03), and thus we get

$$
\text { (21.04) } \begin{aligned}
N_{i}\left(x+h, x_{0} ; g\right)= & \sum_{s=0}^{\infty} \frac{B_{s}(h)}{\pi} \int_{x_{0}}^{x_{0}+b} g(t)\left(\int_{\mathbb{C}_{r}^{\prime+)}} \lambda^{s-k} e^{\lambda(x-t)} d \lambda\right) d t \\
& +\sum_{s=0}^{\infty} \frac{B_{s}(h)}{\pi} \int_{x_{0}}^{x_{0}+a} g(t)\left(\int_{\mathbb{C}_{r}^{(-)}} \lambda^{s-k} e^{\lambda(x-t)} d \lambda\right) d t
\end{aligned}
$$

Remembering that

$$
\begin{aligned}
& \frac{1}{\pi} \int_{x_{0}}^{x_{0}+b} g(t)\left(\int_{\mathbb{C}_{r}^{(}(+)} \lambda^{l} e^{\lambda(x-t)} d \lambda\right) d t+\frac{1}{\pi} \int_{x_{0}}^{x_{0}++^{\prime}} g(t)\left(\int_{\mathbb{C}_{r}^{(-)}} \lambda^{l} e^{\lambda(x-t)} d \lambda\right) d t \\
& =\frac{d^{l}}{d x^{b}}\left\{\frac{1}{\pi} \int_{x_{0}+d^{2}}^{x_{0}+b} g(t) \frac{\sin \rho_{r}(x-t)}{x-t} d t\right\}, \quad(l \geqq 0),
\end{aligned}
$$

we obtain

(21.06) $\frac{d^{k}}{d x^{k}}\left\{N_{r}\left(x+h, x_{0} ; g\right)\right\}=\sum_{l=0}^{\infty} B_{l}(h) \frac{d^{l}}{d x^{k}}\left\{\frac{1}{\pi} \int_{x_{0}+c_{l}}^{x_{0}+b} g(\eta) \frac{\sin \rho_{r}(x-\eta)}{x-\eta} d \eta\right\}$.

Of course, this formula is merely valid when the cxpansion (21.02) is permitted, that is, when the contour $\mathfrak{S}_{r}$ and therefore the points $\pm i \rho_{r}$ belong to tho circle $|\lambda|<\rho$.

Therefore we are not permitted to suppose that $\rho_{r \rightarrow \infty} \rightarrow \infty$ as $r \rightarrow \infty$. Nevertheless, the formula (21.06) indicates the way how we proceed.

Let us assume that $g(t)$ is indefinitely many times differentiable, and

$$
\frac{d^{l}}{d x^{l}}\left\{\frac{1}{\pi} \int_{x_{0}+a}^{x_{0}+b} g(t) \frac{\sin \rho_{r}(x-t)}{x-t} d t\right\}=g_{r}^{(t)}(x)
$$

and that

$$
\sum_{s=0}^{\infty} B_{s}(h) g_{i}^{(s)}(x)
$$

is uniformly convergent concerning $h$ in $a<h<b$, for any $x$ in $x_{0}+\alpha<x<x_{0}+\beta$, then it follows that

$$
\frac{d^{k}}{d x^{h}}\left\{N_{r}\left(x+h, x_{c} ; g\right)\right\}=\sum_{s=0}^{\infty} B_{s}(h) g_{i}^{s^{\prime}}(x) .
$$

This formal result may be considered as the starting point of the first way, although, for the strict argument, we must proceed somewhat appa. 
rently different course, which we will discuss in the following paragraphs. The second way concerns itself with the above consideration more intimately: it appeals to the dissection of given function $g(x)$ into ranges of the low and the high frequencies, and it was adopted by Wiener [1] in his solution of the difference equation (0.32) by trigonometrical integrals, which we will generalise for the non-homogeneous linear translatable equation (20.01) under certain conditions in another occasion.

The third way starts with the expansion of the meromorphic function $1 / G(\lambda)$ into series of exponential functions, and it was adopted by Bochner [3] in his study of the asymptotic behaviour of the solution of the linear difference equation. We will generalise this method in our definition of the principal solution in $\$ 26$.

In the sequel we shall assume that the interchange of the order of the definite integration and the operator $\Lambda$ is permitted for the functions which aro L-integrable by Property III.

\section{$\$ 22$. Introduction of Generalised Bernoulli's Polynomials.}

Let $\lambda_{0}$ be any assigned point in the complex $\lambda$-plane, and let $\lambda_{\theta}$ be a zero-point of the order $k$ of the generating function $G(\lambda)$; that is,

$$
\begin{array}{ll}
G^{(s)}\left(\lambda_{0}\right)=0, & (s=0,1.2, \ldots, k-1) \\
G^{(k)}\left(\lambda_{3}\right) \neq 0, & (k \geqq 0)
\end{array}
$$

Then we have, by Taylor's expansion of analytic function,

$$
\frac{\left(\lambda-\lambda_{0}\right)^{k} e^{\left(\lambda-\lambda_{0}\right) h}}{G(\lambda)}=\sum_{s=0}^{\infty} B_{s, \lambda_{0}}^{k}(h)\left(\lambda-\lambda_{0}\right)^{*}
$$

which is valid in $\left|\lambda-\lambda_{0}\right|<\rho\left(\lambda_{0}\right), \rho\left(\lambda_{0}\right)$ being the distance between $\lambda_{0}$ and the nearest zero-points of $G(\lambda)$ to $\lambda_{0}$.

Let it be noticed that $\left\{B_{s, \lambda_{a}}^{*}(h)\right\}$ are the polynominals of the degree $s$, and that if specially

$$
\Gamma f(x) \equiv f(x+1)-f(x)
$$

and

$$
\lambda_{0}=0
$$

$\left\{B_{s_{,} \lambda_{\theta}}^{k}(h)\right\}$ become the classical Bernoulli's polynomials. Therefore we will give

Definition X. The polynomials $\left\{B_{s, \lambda_{0}}^{k}(h)\right\}$ are called the generalised Bernoulli's polynomials of the linear translatable operator $\Gamma$ adjointed to the point $\lambda_{0}$

These polynomials $\left\{B_{s, \lambda_{0}}^{k}(h)\right\}$ possess the following fundamental properties which are analogons to those of the ordinary Bernonlli's polynomial. 
Theorem XX.I. We have

(22.05) $\Gamma B_{s, \lambda_{0}}^{\mathrm{c}}(h) e^{\lambda_{0} h} \equiv \Gamma_{\xi}\left\{B_{s, \lambda_{0}}^{x_{i}}(h+\xi) e^{\lambda_{0}(h+\xi)}\right\}=\left\{\begin{array}{cc}\frac{h^{s-l i}}{(s-k) !} e^{\lambda_{0} h},(s=k, k+1, \ldots) \\ 0, \quad(s=0, \ldots, k-1)\end{array}\right.$

$$
-\frac{d}{d h}\left\{B_{s, \lambda_{0}}^{k}(h)\right\}=B_{s-1, \lambda_{0}}^{k}(h), \quad(s=1,2, \ldots),
$$

and

$$
B_{s, \lambda_{0}}^{k}\left(h_{1}+h_{2}\right)=\sum_{\nu=0}^{s} \frac{h_{2}{ }_{\nu}}{\nu !} B_{s-\nu, \lambda_{0}}^{k}\left(h_{1}\right), \quad(s=0,1,2, \ldots) .
$$

To prove (22.05), let us write, in view of (22.02),

$$
\frac{\left(\lambda-\lambda_{0}\right)^{k} e^{\lambda h}}{G(\lambda)}=\sum_{s=0}^{\infty} B_{s, \lambda_{0}}^{k}(h) e^{\lambda_{0} \pi^{n}}\left(\lambda-\lambda_{0}\right)^{s} .
$$

Operating $\Gamma$ to both sides, we have

$$
\left(\lambda-\lambda_{0}\right)^{k} e^{\lambda h}=\sum_{s=0}^{\infty} \Gamma_{0}\left\{B_{s, \lambda_{0}}^{k}(h+\xi) e^{\lambda_{0}(h+\xi)}\right\}\left(\lambda-\lambda_{0}\right)^{s},
$$

which proves (22.05).

To see (22.06), differentiate both sides of (22.02) with respect to $h$, and then compare their coefficients respectively.

To get (22.07), we have only to remind, in view of (22.02),

that is,

$$
\frac{\left(\lambda-\lambda_{1}\right)^{k} e^{\left(\lambda-\lambda_{0}\right)\left(h_{1}+h_{2}\right)}}{G(\lambda)}=e^{\left(\lambda-\lambda_{0}\right) h_{2}} \frac{\left(\lambda-\lambda_{\theta}\right)^{k} e^{\left(\lambda-\lambda_{0}\right) h_{1}}}{G(\lambda)},
$$

$(22.11$

$$
\sum_{s=0}^{\infty} B_{s, \lambda_{0}}^{k}\left(h_{1}+h_{2}\right)\left(\lambda-\lambda_{0}\right)^{s}=\sum_{s=0}^{\infty} \frac{h_{2}^{s}\left(\lambda-\lambda_{0}\right)^{s}}{s !} \sum_{s=0}^{\infty} B_{s, \lambda_{0}}^{k}\left(h_{1}\right)\left(\lambda-\lambda_{0}\right)^{s} \text {. (q. e. d.) }
$$

We will now expand $G(\lambda)$ in the point $\lambda_{0}$. Then by the assumption (22.01), we may put

$$
G(\lambda)=\sum_{s=k}^{\infty} \frac{G_{s, \lambda_{0}}^{k}}{s !}\left(\lambda-\lambda_{\theta}\right)^{s} .
$$

Theorem XXII. II. We have

$$
\Gamma_{0}\left\{\xi^{p} e^{\lambda_{0} \xi}\right\}= \begin{cases}G_{\nu, \lambda_{0}}^{k}, & (\nu=k, k+1, k+2, \ldots), \\ 0, & (\nu=0,1, \ldots, k-1),\end{cases}
$$

and

$$
\sum_{\nu \sim 0}^{s} \frac{G_{k+s-\nu, \lambda_{0}}^{k}}{(k+s-\nu) !} B_{\nu, \lambda_{0}}^{k}(h)=\frac{h^{s}}{s !} .
$$

Proof. By definitions we have

$$
\begin{aligned}
G(\lambda) & =\Gamma_{0}\left\{e^{\lambda \varepsilon}\right\} \\
& =\Gamma_{\xi}\left\{e^{\lambda_{0} \xi} \sum_{s=0}^{\infty} \frac{\xi^{s}\left(\lambda-\lambda_{0}\right)^{s}}{s !}\right\}
\end{aligned}
$$




$$
=\sum_{s=0}^{\infty} \frac{\left(\lambda-\lambda_{0}\right)^{s}}{s !} \Gamma_{\theta}\left\{e^{\lambda_{0} \xi} \xi^{s}\right\}
$$

Comparing this last power series with (22.12), we get (22.13).

To prove (22.14), write, in view of (22.02)

$$
\left(\lambda-\lambda_{0}\right)^{k} e^{\left(\lambda-\lambda_{0}\right) h}=\sum_{s=k}^{\infty} \frac{G_{s, \lambda_{0}}^{k}}{s !}\left(\lambda-\lambda_{0}\right)^{s} \sum_{j=0}^{\infty} B_{j, \lambda_{0}}^{k}(h)\left(\lambda-\lambda_{0}\right)^{j},
$$

and then compare the corresponding coefficients of both sides. (q.e.d.)

Before we give a certain generalisation of Euler-Maclaurin's theorem, we will prepare ourselves with the following:

Lemma XXII.I. Let $g(x)$ be $m+1$-time differentiable in the interval $(a, b)$, and let $g^{(m+1)}(x)$ be Lebesgue-integrable there.

If we denote by $D_{x}$ differentiation with respect to $x$, then, by usual symbolic notation, we have

$$
\begin{aligned}
g(x+h) & =\sum_{\nu=0}^{m} \frac{h^{v}}{\nu !} e^{\lambda_{0} l}\left(D_{x}-\lambda_{*}\right)^{\nu} g(x) \\
& +\int_{0}^{h} \frac{(h-t)^{n}}{m !} e^{\lambda_{0}\left(h_{n}-t\right)}\left(D_{x}-\lambda_{0}\right) g^{m+1}(x+t) d t
\end{aligned}
$$

provided that $x$ and $x+h$ belong to $(a, b)$.

To prove this, let us consider an auxiliary function

$$
\psi(x)=g(x) e^{-\lambda_{0} x} .
$$

The well-known mean-value theorem yields us

$$
\psi(x+h)=\sum_{i=0}^{m} \frac{h^{\nu}}{\nu !} \psi^{(\nu)}(x)+\int_{c}^{h} \frac{(h-t)^{m}}{m !} \psi^{(m+1)}(x+t) d t
$$

Here we have

$$
\psi^{(v)}(x)=e^{-\lambda x_{0}}\left(D_{x}-\lambda_{i}\right)^{v} g(x), \quad(v=0,1,2, \ldots)
$$

Putting these into (22.19), we obtain (22.17), which is to be proved.

Theorem XXII. III. (Generalisation of Euler"Maclaurin's theorem) Let $g(x)$ be $m+k+1$-time differentiable in the interval $(a, b)$, and let $g^{(m+k+1)}(x)$ be Lebesgue-integrable there.

If we put

$(22.21)$

$$
\left(D_{n}-\lambda_{i}\right)^{k} \phi(x)=g(x)
$$

then we have

$$
\begin{aligned}
g(x+h) & =\sum_{s=01}^{m+k} B_{i, \lambda_{0}}^{k}(h) e^{\lambda_{0} h} \Gamma_{0}\left\{\left(D_{x}-\lambda_{0}\right)^{s} \phi(x+\xi)\right\} \\
& +\Gamma_{\xi}\left\{\int_{\xi}^{h} e^{\lambda_{0}(h+\xi-\eta)} B_{m+\lambda_{i}, \lambda_{0}}^{k}(h+\xi-\eta)\left(D_{x}-\lambda_{1}\right)^{m+1} g(x+\eta) d \eta\right\},
\end{aligned}
$$

provided that $x$ and $x+h$ belong to $(a, b)$. 
Proof. In view of (22.14), (22.17) may be written, with respect to $g(x+h)$, in the following form:

$$
\begin{aligned}
g(x+h)= & \sum_{s=0}^{m} \sum_{v=0}^{s} \frac{G_{k+s \cdot v, \lambda_{0}}^{k}}{(k+s-\nu) !} B_{\nu, \lambda_{0}}^{k}(h) e^{\lambda_{0} h}\left(D_{x}-\lambda_{0}\right)^{v} g(x) \\
& +\int_{0}^{h} \frac{(h-t)^{m}}{m !} e^{\lambda_{0} \cdot h-t,}\left\{\left(D_{x}-\lambda_{0}\right)^{m_{-1} \gamma} g(x+t)\right\} d t
\end{aligned}
$$

But we have, by rearrangement of terms and (22.21),

$$
\begin{aligned}
g(x+h)= & \sum_{s=0}^{m} B_{s, \lambda_{0}}^{k}(h) e^{\lambda_{0} h} \sum_{t \sim s}^{m} \frac{C_{*+t-s, \lambda_{0}}^{k}}{(k+t-s) !}\left(D_{x}-\lambda\right)^{t+k} \phi(x) \\
& +\int_{0}^{h} \frac{(h-t)^{\prime}}{m !} e^{\lambda_{0}(h-t)}\left\{\left(D_{x}-\lambda_{0}\right)^{m+1} g(x+t)\right\} d t .
\end{aligned}
$$

Here, in view of (22.13) and (22.17),

$$
\begin{aligned}
& \Gamma_{\xi}\{\phi(x+\xi)\}=\sum_{\sigma=k}^{m+k-s} \frac{\left(G_{\sigma, \lambda_{0}}^{k}\right.}{\sigma !}\left(D_{x}-\lambda_{i}\right)^{\sigma} \phi(x) \\
& +\Gamma_{\xi}\left\{\int_{0}^{\xi / \xi-\eta)^{m+k-s}} \frac{\xi(k+k) !}{\left(m+\lambda_{0}(\xi-\eta)\right.}\left\{\left(D_{x}-\lambda_{0}\right)^{m+k-s+1} f(x+\eta)\right\} d \eta\right\} .
\end{aligned}
$$

Therefore we have

$$
\begin{aligned}
\sum_{t-s}^{m} \frac{G_{k+t-s, \lambda_{0}}^{k}}{(k+t-s) !}\left(D_{x}-\lambda_{*}\right)^{t+k} \phi(x) & \\
= & \sum_{\sigma=k}^{m+k-s} \frac{G_{\sigma, \lambda_{0}}^{k}}{\sigma !}\left(D_{x}-\lambda_{0}\right)^{\sigma+s} \phi(x) \\
= & \Gamma_{0}\left\{\left(D_{x}-\lambda_{0}\right)^{s} \phi(x+\xi)\right\} \\
& -\Gamma_{0}\left\{\int_{0}^{\xi} \frac{(\xi-\eta)^{m+k-s}}{(m+k-s) !} e^{\lambda_{0} \xi-\eta !}\left\{\left(D_{x}-\lambda_{0}\right)^{m+k+1} \phi(x+\eta)\right\} d \eta\right\} .
\end{aligned}
$$

Combining (22.23)-(22.26), we obtain

(22.27) $\quad g(x+h)$

$$
\begin{aligned}
& =\sum_{s=0}^{m} B_{s, \lambda_{0}}^{k}(h) e^{\lambda_{0} h} \Gamma_{\xi}\left\{\left(D_{x}-\lambda_{0}\right)^{s} \phi(x+\xi)\right\} \\
& -\sum_{s=0}^{m} B_{s, \lambda_{0}}^{k}\left(h e^{\lambda_{0} h} \Gamma_{0}\left\{\int_{0}^{\vdots} \frac{(\xi-\eta)^{m+k-s}-e^{\lambda_{0}(\xi-\eta)}}{(m+k-s) !}\left\{\left(D_{x}-\lambda_{0}\right)^{m+k+1} \phi(x+\eta)\right\} d \eta\right\}\right. \\
& +\int_{0}^{h} \frac{(h-t)^{m}}{m !} e^{\lambda_{0}(h-t)}\left\{\left(D_{x}-\lambda_{0}\right)^{m+1} \varphi(x+t)\right\} d t
\end{aligned}
$$

where the second term may be written

$$
\text { (22.28) } \sum_{s=0}^{m} B_{s, \lambda_{0}}^{k}(h) e^{\lambda_{0} h} \Gamma_{0}\left\{\int_{0}^{\xi} \frac{(\xi-\eta)^{m+k-s} e^{\lambda_{0}(\xi-\eta)}}{(m+k-s) !}\left\{\left(D_{x}-\lambda_{0}\right)^{m_{2+k+1}} \phi(x+\eta)\right\} d \eta\right\}
$$




$$
\begin{aligned}
& =\Gamma_{\xi}\left\{\int_{0}^{\xi} e^{\lambda_{\theta}(h+\xi-\eta)} \sum_{s=0}^{m} B_{s, \lambda_{0}}^{k}(h) \frac{(\xi-\eta)^{m+k-s}}{(m+k-s) !}\left\{\left(D_{s}-\lambda_{0}\right)^{m+k+1} \phi(x+\eta)\right\} d \eta\right\} \\
& =\mathrm{I}_{\boldsymbol{\nu}},
\end{aligned}
$$

say. Here let it be noticed that

(22.29)

$$
\begin{aligned}
& \int_{0}^{h} \frac{(h-t)^{m}}{m !}\left\{\left(D_{x}-\lambda_{0}\right)^{m+1} g(x+t)\right\} d t \\
& \quad=\int_{0}^{h} \Gamma_{\xi}\left\{e^{\lambda_{0}(h+\xi-t)} B_{m+k, \lambda_{0}}^{k}(h+\xi-t)\right\}\left\{\left(D_{x}-\lambda_{0}\right)^{m+1} g(x+t)\right\} d t \\
& \quad=\Gamma_{\xi}\left\{\int_{0}^{h} e^{\lambda_{0}(h+\zeta-t)} B_{m+k, \lambda_{0}}^{k}(h+\zeta-t)\left\{\left(D_{x}-\lambda_{1}\right)^{m+1} g(x+t)\right\} d t\right\} .
\end{aligned}
$$

Further, by using Lemma XXIi. 1.,

$$
\begin{aligned}
& \sum_{s=0}^{m} B_{s, \lambda 0}^{k}(h) \frac{(\xi-\eta)^{m+k-s}}{(m+k-s) !} \\
& \quad=B_{m+k, \lambda_{0}}^{k}(h+\xi-\eta)-\sum_{s=m+1}^{m+k} B_{s, \lambda_{0}}^{k}(h) \frac{(\xi-\eta)^{m+k-s}}{(m+k-s) !} .
\end{aligned}
$$

Thus we have

$$
\text { (22.31) } \begin{aligned}
\mathrm{I}_{2} & =\Gamma_{0}\left\{\int_{0}^{\xi} e^{\lambda_{0}(h+\xi-\eta)} B_{m+k, \lambda_{0}}^{k}(h+\xi-\eta)\left\{\left(D_{x}-\lambda_{0}\right)^{m+1} g(x+\eta)\right\} d \eta\right\} \\
& -\sum_{s=m+1}^{m+k} B_{s, \lambda_{0}}^{k}(h) \Gamma_{0}\left\{\int_{0}^{\xi} e^{\lambda_{0}(h+\xi-\eta)} \frac{(\xi-\eta)^{m+k-s}}{(m+k-s) !}\left\{\left(D_{x}-\lambda_{0}\right)^{m+k+1} \phi(x+\eta)\right\} d \eta\right\} .
\end{aligned}
$$

Combining (22.28)-(22.31), we establish

(22.32) $g(x+h)$

$$
\begin{aligned}
& =\sum_{s=0}^{m} B_{s, \lambda_{0}}^{k}(h) e^{\lambda_{0} h} \Gamma_{0}\left\{\left(D_{x}-\lambda_{0}\right)^{s} \phi(x+\xi)\right\} \\
& +\sum_{s=m+1}^{m+k} B_{s, \lambda_{0}}^{k}(h) e^{\lambda_{0} h} \Gamma_{0}\left\{e^{\lambda_{0} \xi} \int_{0}^{\xi} e^{-\lambda_{0} \eta} \frac{(\xi-\eta)^{m+k-s}}{(m+k-s) !}\left\{\left(D_{x}-\lambda_{0}\right)^{m+k+1} \phi(x+\eta)\right\} d \eta\right\} \\
& -\Gamma_{0}\left\{\int_{0}^{\xi} e^{\lambda_{0}(h+\xi-\eta)} B_{m+k, \lambda_{0}}^{k}(h+\xi-\eta)\left\{\left(D_{x}-\lambda_{0}\right)^{m+1} g(x+\eta)\right\} d \eta\right\} \\
& +\Gamma_{0}\left\{\int_{0}^{h} e^{\lambda_{0}(h+\xi-\eta)} B_{m+k, \lambda_{0}}^{k}(h+\xi-\eta)\left\{\left(D_{x}-\lambda_{0}\right)^{m+1} g(x+\eta)\right\} d \eta\right\},
\end{aligned}
$$

which leads us to (22.22) (g.e.d.).

\section{$\S 23$. Linear Translatability and $\phi$-polynomials of L. M. Milne-Thomson's.}

L. M. Milne-Thomson [1] defined $\phi$-polynomials $\phi_{\nu}^{(n)}(x)$ of degree $\nu$ and order $n$ by the relation

$$
f(\lambda, n) e^{x \lambda+y(\lambda)}=\sum_{\nu=0}^{\infty} \frac{\lambda^{\nu}}{\nu !} \phi_{\nu}^{(n)}(x),
$$


where $f(\lambda, n)$ and $g^{\prime}(t)$ are such that for a certain range of $x$ the expansion on the right exists as the uniformly convergent series of $t$.

We will show certain relations between these $\phi$-polynomialis and our generalised Bernoulli's polynomials.

Let an operator $\Gamma^{(n)}$ be defined by

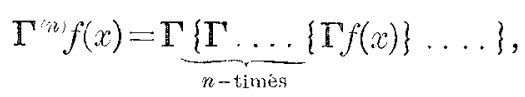

that is to say, let $\Gamma^{(n)}$ be $n$-time iterated linear functional operator of $\Gamma$.

As easily vorified, $\Gamma^{(n)}$ is also translatable, and its generating function, which we denote by $G_{n}(\lambda)$, is given by

$$
G_{n}(\lambda)=G(\lambda)^{n}
$$

By the assumption (22.01), we may put

$$
\frac{\left(\lambda-\lambda_{n}\right)^{m n} e^{\lambda x}}{G(\lambda)^{n}}=\sum_{s=m n}^{\infty} B_{s, \lambda_{0}}^{(m), n}(x) e^{\iota_{0} r}\left(\lambda-\lambda_{0}\right)^{s} .
$$

Thus we will give

Definition XI. The polynomials $\left\{B_{s, \lambda_{0}}^{(m, n)}(x)\right\}$ defined by (23.04) are called the generalised Bernoulli's polynomials of degree $s$ and order $n$ at the point $\lambda$.

Thus these $B_{s, \lambda_{0}}^{(m), n}(x)$ are $\phi$-polynomials, and let it be noticed that by the linear translatability the choice of $f(\lambda, t)$ has a clear meaning in this case.

\section{§24. Boundary Value Problem of Linear Homogeneous Functional Equation and Introduction of $\left\{\dot{B}_{s, \lambda_{0}}^{k}(x)\right\}$.}

By virtue of the convergence-theorem in $\$ 3$, we are able to establish the following:

Theorem XXIV. I. Let us assume that there is a sequence of contours $\left\{\mathfrak{C}_{r}\right\}$ such that

$$
\left\{\mathfrak{S}_{i}\right\} \in \mathbb{S}\left(\delta_{i},-\delta_{2}\right)
$$

and further, with respect to this sequence

$$
G(\lambda)=\mathbb{E}_{i},-O\left(s ; a+\delta_{1}{ }^{\prime}, b-\delta_{2}{ }^{\prime}\right),
$$

where we may take positive numbers $\delta_{1}, \delta_{1}, \delta_{2}$ and $\delta_{2}^{\prime}$, as small as we please.

Let $b(x)$ be a functions which is defined in the open interval $(a, b)$ and belongs to $\left(\mathscr{L}_{s}\right)(a, b)$, where $s$ is subject to $s \geqq n$.

If the contour-integrals $\mathrm{T}_{r}(x, 0 ; b)$ defined for this sequeuce of contours have the limiting function $\dot{b}(x)$ to which they tend uniformly in any finite interval of the $x$-axis, then

$$
\dot{b}(x)=b(x), \quad a<x<b,
$$


and

$$
\Gamma \dot{b}(x)=0, \quad-\infty<x<\infty .
$$

To prove this, we have only to remind Theorem III.I. and Theorem VI.II.

Further, combining Theorem III.II. and Theorem VI.II., we may obtain

Theorem XXIV.II. If $b(x)$ is defined in the open interval $(a, b)$, and belongs to $\left(V_{s}\right)(a, b)$, then we may replace the assumption (24.02) to Theorem XXIV.I, by the weaker assumption

$$
G(\lambda)=\mathfrak{c}_{r}-o\left(s+1 ; a+\delta_{1}{ }^{\prime}, b-\delta_{2}{ }^{\prime}\right)
$$

where $\delta_{1}^{\prime}$ and $\delta_{3}^{\prime}$ may be any given positive number, and the conclusion to Theorem XXIV.I. remains unchanged.

Thus under certain conditions we have proved the existence of the solution of the functional equation (24.04) which satisfies the boundary condition (24.03).

The application of the convergence-theorem in $\$ 3$ will give us the following several theorems in this connection.

Theorem XXIV.III. Cnder the assumptions in Theorem XXIV.I. or Theorem XXIV.II., let there be a function $\beta(x)$ which satisfies (24.03) and (\$4.04) and which belongs to $\left(\mathscr{L}_{s}\right)\left(x_{0}+a, x_{0}+b\right)$ or $\left(\mho_{s}\right)\left(x_{0}+a, x_{0}+b\right)$ respectively, where $x_{0}$ is any real number and $s$ is greater than $n$.

Then we have

$$
\beta(x)=b(x), \quad-\infty<x<\infty
$$

Proof. Let us assume that the assumptions in Theorem XXIV I. are satisfied. Then ince $\beta(x)$ belongs to $\left(V_{s}\right)\left(x_{0}+\alpha, x_{0}+b\right)$ for any real $x_{0}$, the application of Theorem III.I. will give us

$$
\beta(x)=\lim _{r \rightarrow \infty} \mathrm{T}_{r} .\left(x, x_{0} ; \beta\right), \quad\left(x_{0}+a<x<x_{0}+\beta\right) .
$$

But since $\beta(x)$ is a solution of (24.04), we have, for any $x$ and $x_{n}$,

$$
\mathrm{T}_{r}\left(x, x_{n} ; \beta\right)=\mathrm{T}_{r}(x, 0 ; \beta),
$$

and by the assumption (24.03)

$$
\mathrm{T}_{r}(x, 0 ; \beta)=\mathrm{T}_{r}(x, 0 ; b) .
$$

Thus we get

$$
\begin{aligned}
\beta(x) & =\lim _{r \rightarrow \infty} \mathrm{T}_{r}(x, 0 ; \beta)=\lim _{r \rightarrow \infty} \mathrm{T}_{r}(x, 0 ; b) \\
& =\dot{b}(x),
\end{aligned}
$$

which was to be proved.

The remaining part of the theorem may be proved quite similarly.

Theorem XXIV.IV. In the assumptions to Theorem XXIV.I. or 
Theorem XXIV.II., let us replace (24.02) or (24.05) by

$$
G(\lambda)=\mathfrak{S}_{r}-O\left(m ; a+{ }_{1}^{\prime}, b-\delta_{2}^{\prime}\right)
$$

$o r$

$$
G(\lambda)=\mathfrak{E}_{r}-o\left(m+1 ; a+\delta_{1}{ }^{\prime}, b-\delta_{2}{ }^{\prime},\right.
$$

respectively.

Then the corresponding $\dot{b}(x)$ is $m-s$ time differentiable in $(-\infty, \infty)$.

This may be also proved by convergence-theorem in $\$ 3$.

In the sequel we need the solution of (24.04) specially when

$$
b(x)=B_{s, \lambda_{0}}^{k}(x) e^{\lambda_{0} x} .
$$

For this object we will begin with the following:

Lemma XXIV.I. If we take $\mathfrak{S}_{\lambda_{0}}$ around the point $\lambda_{0}$ with sufficicntly small radius such that the distance between $\mathfrak{E}_{\lambda_{0}}$ and $\mathfrak{E}_{r}$ is positive, then, putting

$$
b(x)=B_{s, \lambda_{0}}^{k}(x) e^{\lambda_{0} x},
$$

we have

$$
\begin{aligned}
\mathrm{S}_{r}(x, 0 ; b) \equiv & \frac{1}{2 \pi i} \oint_{\mathscr{E}_{r}} \frac{e^{\lambda x}}{G(\lambda)} \Gamma_{\xi}\left\{e^{\lambda \varepsilon} \int_{0}^{\xi} B_{s, \lambda 0}^{k}(\eta) e^{\lambda_{0} \eta} e^{-\lambda \eta} d \eta\right\} d \lambda \\
= & -\frac{1}{2 \pi i} \oint_{\mathscr{E}_{r}} \frac{e^{\lambda x}}{\left(\lambda-\lambda_{0}\right)^{\varepsilon-k+1} G(\lambda)} d \lambda \\
& +\frac{1}{2 \pi i} \oint_{\mathscr{E}_{\lambda_{0}}} \frac{e^{\lambda x}}{\left(\lambda-\lambda_{0}\right)^{s-k+1} G(\lambda)} d \lambda,
\end{aligned}
$$

To prove this, we notice that, by suitable choice of $\mathfrak{E}_{\lambda_{0}}$, we have

$$
B_{s, \lambda_{0}}^{k}(h) e^{\lambda_{0} h}=\frac{1}{2 \pi i} \oint_{\mathscr{E}_{\lambda_{0}}} \frac{\left(z-\lambda_{0}\right)^{k} e^{h z}}{\left(z-\lambda_{0}\right)^{i+1} G(z)} d z,
$$

which is possible by (22.08). Therefore we may and we shall write

$$
\begin{aligned}
& \Gamma_{0}\left\{e^{\lambda \xi} \int_{0}^{\xi} B_{s, \lambda_{0}}^{l}(\eta) e^{\lambda_{0} \eta} \cdot e^{-\lambda \eta} d \eta\right\} \\
& =\Gamma_{\xi=\xi}\left\{e^{\backslash \xi} \int_{0}^{\xi}\left(\frac{1}{2 \pi i} \oint_{\mathfrak{E}_{\lambda_{0}}} \frac{\left(z-\lambda_{0}\right)^{k} e^{\eta(z+\lambda)}}{\left(z-\lambda_{0}\right)^{s+1} G(z)} d z\right) d \eta\right\} \\
& =\frac{1}{2 \pi i} \oint_{\mathbb{E}_{\lambda_{0}}} \frac{\left(z-\lambda_{0}\right)^{k} \Gamma_{\xi=1}\left\{e^{\lambda \xi} \int_{0}^{\xi} e^{\mu(z-\lambda)} d \eta\right\}}{\left(z-\lambda_{0}\right)^{+1+} G(z)} d z
\end{aligned}
$$




$$
=\frac{1}{2 \pi i} \oint_{\mathfrak{E}_{\lambda_{0}}} \frac{G(z)-G(\lambda)}{\left(z-\lambda_{0}\right)^{s+1-k} G(z)(z-\lambda)} d z .
$$

Consequently we have

$$
\begin{aligned}
& S_{i}(x, 0 ; \beta) \\
& =\frac{1}{2 \pi i} \oint_{\mathscr{E}_{i}} \frac{e^{\lambda x}}{G(\lambda)}\left\{\frac{1}{2 \pi i} \oint_{\mathfrak{E}_{\lambda_{0}}} \frac{d z}{\left(z-\lambda_{0}\right)^{s+1-k_{0}}(z-\lambda)}\right\} d \lambda \\
& -\frac{1}{2 \pi i} \oint_{\mathcal{E}_{i}} e^{\lambda x}\left\{\frac{1}{2 \pi i} \oint_{\Theta_{\lambda_{0}}} \frac{d z}{\left(z-\lambda_{0}\right)^{s+1-k}(z-\lambda) G(z)}\right\} d \lambda,
\end{aligned}
$$

which yields us our lemma.

This Lemma will lead us to the following:

Definition XII. If there is a sequence of contours $\left\{\mathfrak{S}_{r}\right\}$ such that $\mathrm{S}_{r}(x, 0 ; \beta)$ defined in Lemma XXIV.I is uniformiy convergent in any finite range of $x$-axis for some fixed $s$, then the limiting function will be denoted, by $\dot{B}_{s, \lambda_{0}}^{k}(x)$.

Direct applications of the theorems obtained in this paragraph will now give us

Theorem XXIV.V. If the function $i_{s, \lambda_{0}}^{k}(x)$ exists for some integer $s$, then there is auniquely determined integer $s\left(\lambda_{0}\right)$ with the following properties:

$1^{\circ} \cdot \dot{B}_{\nu, \lambda_{0}}^{i}(x)$ exists for any integer $v$ subject to $\nu \geqq s\left(\lambda_{0}\right)$, but $\dot{B}_{v, \lambda_{0}}^{k}(x)$ does not exist for $\nu \leqq s\left(\lambda_{\theta}\right)-1$.

$2^{\circ}$. If $\nu \geqq s\left(\lambda_{*}\right)+1$, then we have

$$
\begin{aligned}
\left(D_{x}-\lambda_{0}\right) \dot{B}_{v, \lambda_{0}}^{k}(x) & =\dot{B}_{\nu-1, \lambda_{0}}^{k}(x) . & & \\
\dot{B}_{\nu, \lambda_{0}}^{k}(x) & =B_{v, \lambda_{0}}^{k}(x) e^{\lambda_{0} *}, & & (a<x<b)\left(\nu \geqq s\left(\lambda_{0}\right)\right) . \\
\Gamma \dot{B}_{v, \lambda_{0}}^{*}(x) & =0, & & (-\infty<x<\infty)\left(\nu \geqq s\left(\lambda_{0}\right)\right) .
\end{aligned}
$$$$
3^{\circ} \text {. }
$$

\section{§25. Summation Formula.}

Since we have defined $\left\{\dot{B}_{r, \lambda_{0}}^{k}(x)\right\}$, there is no difficulty to obtain the following fundamental

Theorem XXV.I. (Summation Formula).

Let $g(x)$ be defined for $(x+a, \infty)$, and let it be $m+1$-times differentiable there, where $m$ is a non-negative integer not less than $s\left(\lambda_{0}\right)$.

Let the existence and the equality of two functions of $x$

and

$$
\Gamma_{\xi}\left\{\int_{\xi}^{\infty} \dot{B}_{m+k, \lambda_{0}}^{k}(h+\xi-\eta)\left\{\left(D_{x}-\lambda_{0}\right)^{m+1} g(x+\eta)\right\} d \eta\right\},
$$

$(25.02)$

$$
\int_{0}^{\infty} \Gamma_{0}\left\{\dot{B}_{m+k, \lambda_{0}}^{i}(h+\xi-\eta)\right\}\left\{\left(D_{x}-\lambda_{0}\right)^{m+1} g(x+\eta ; d \eta\right.
$$


be assumed.

Then putting, as in (22.21),

$$
g(x)=\left(D_{x}-\lambda_{0}\right)^{k} \phi(x),
$$

we have

$$
\begin{aligned}
g(x+h)= & \sum_{s=0}^{m+k} B_{s, \lambda_{0}}^{k}(h) e^{\lambda_{0} / h} \Gamma_{0}\left\{\left(D_{x}-\lambda_{0}\right)^{s} \phi(x+\xi)\right\} \\
& +\int_{0}^{\infty} \dot{B}_{m+\tilde{k}, \lambda_{0}}^{k}(h-t) \underset{0}{\Gamma_{\xi}}\left\{\left(D_{x}-\lambda_{0}\right)^{m+1} g(x+\xi+t)\right\} d t
\end{aligned}
$$

To prove this, we appeal to Theorem XXII.III. which yields us

$$
\begin{aligned}
g(x+h)= & \sum_{s=0}^{m+k} B_{s_{1} \lambda_{0}}^{k}(h) e^{\lambda_{0} h} \Gamma_{\xi}\left\{\left(D_{n}-\lambda_{0}\right)^{s} \phi(s+\xi)\right\} \\
& +\Gamma_{0}\left\{\int_{\xi}^{h} e^{\left.\lambda_{0} h+\xi-\eta\right)} B_{m+k, \lambda_{0}}^{c}(h+\xi-\eta)\left\{\left(D_{x}-\lambda_{0}\right)(s+\eta)\right\} d \eta\right\} .
\end{aligned}
$$

Let us put, for the sake of brevity,

$$
\text { (25.06) } \Gamma_{0}\left\{\int_{\xi}^{n} e^{\lambda_{0}(l b+\xi+\eta} B_{m+k, \lambda_{0}}^{k}(h+\xi-\eta)\left\{\left(D_{x}-\lambda_{0}\right)^{m+1} g(x+\eta)\right\} d \eta\right\}=R_{m+1}^{k}
$$

Substituting (24.03) in this, and writing $\int_{\xi}^{h}=\int_{0}^{h}+\int_{\xi}^{0}$, we have

$$
\begin{aligned}
R_{n+1}^{k}= & \Gamma_{0}\left\{\int_{0}^{h} \dot{B}_{m+k, \lambda_{0}}^{k}(h+\xi-\eta)\left\{\left(D_{x}-\lambda_{0}\right)^{n+1} g(x+\eta)\right\} d \eta\right\} \\
& +\Gamma_{0}\left\{\int_{\xi}^{0} \dot{B}_{m+k, \lambda_{0}}^{k}(h+\xi-\eta)\left\{\left(D_{x}-\lambda_{0}\right)^{m+1} g(x+\eta)\right\} d \eta\right\} \\
= & \left.\int_{0}^{h} \Gamma_{0}\left\{\dot{B}_{m+k, \gamma_{0}}^{k}(h+\xi-\eta)\right\}\left\{\left(D_{x}-\lambda_{0}\right)^{m+1} g(x+\eta)\right\} d \eta\right\} \\
& +-\Gamma_{\xi}\left\{\int_{\xi}^{0} \dot{B}_{m+k, \lambda_{0}}^{k}(h+\xi-\eta)\left\{\left(D_{x}-\lambda_{0}\right)^{m+1} g(x+\eta)\right\} d \eta\right\},
\end{aligned}
$$

where the first term of the last right-hand side is equal to zero by (24.20) $4^{\circ}$, and the second term may be written, by the assumptions (25.01) and (25.02). as follows:

$$
\begin{aligned}
\Gamma_{0}\left\{\int_{\xi}^{0} \dot{B}_{m+k, \lambda_{0}}^{k}(h+\xi-\eta)\left\{\left(D_{x}-\lambda_{0}\right)^{m+1} g(x+\eta)\right\} d \eta\right\} \\
=\Gamma_{\xi}\left\{\int_{0}^{\infty} \dot{B}_{m+k, \lambda_{0}}^{k}(h+\xi-\eta)\left\{\left(D_{x}-\lambda_{0}\right)^{m+1} g(x+\eta)\right\} d \eta\right\} \\
\quad+\Gamma_{\xi}\left\{\int_{\xi}^{\infty} \dot{B}_{m+k, \lambda_{0}}^{k}(h+\xi-\eta)\left\{\left(D_{x}-\lambda_{0}\right)^{m+1} g(x+\eta)\right\} d \eta\right\} \\
=\int_{0}^{\infty} \Gamma_{\xi}\left\{\dot{B}_{m+k, \lambda_{0}}^{k}(h+\xi-\eta)\right\}\left\{\left(D_{x}-\lambda_{0}\right)^{m+1} g(x+\eta)\right\} d \eta
\end{aligned}
$$




$$
+\Gamma_{0}\left\{\int_{\xi}^{\infty} \dot{B}_{m+k, \lambda_{0}}^{k}(h+\xi-\eta)\left(D_{x}-\lambda_{0}\right)^{m+1} g(x+\eta) d \eta\right\},
$$

where the first term of the right-hand side is equal to zero.

Combining (25.05)-(25.08), we get (2504), which was to be proved.

\section{§26. Principal Solution of Non-homogeneous Linear Translatable Functional Equation.}

We now come to the situation to extend Nörlund's idea of the principal solution to a general non-homogeneous linear translatable functional equation.

Let the equation be given by

$$
\Gamma f(x)=g(x)
$$

for every $x$ which belongs to $(0, \infty)$.

We introduce three families of functions $\{g(x, \lambda)\}_{\lambda_{0}},\left\{H_{\lambda_{0}}(x, \lambda)\right\}_{\lambda_{0}}$ and $\{F(x, \lambda)\}_{\lambda_{0}}$ in the following manner:

$1^{\circ}$. Let a family of functions $g(x, \lambda)$ be defined for the set $\Re_{\lambda_{0}}$ of the parameter $\lambda$ which has $\lambda_{0}$ as its accumulation-point, and let

$$
\lim _{\lambda \rightarrow \lambda_{0}} g\left(x, \lambda_{\vartheta}\right)=g(x), \quad(0 \leqq x<\infty) .
$$

$2^{\circ}$. Let us assume that there is a sequeence of linear translatable functional operators $\left\{\Gamma_{n}\right\}$ such that the series

$$
\sum_{n=0}^{\infty} \Gamma_{n, \xi}\{g(x+\xi, \lambda)\}\left(\equiv \sum_{n=0}^{\infty} \Gamma_{n} g(x, \lambda)\right)
$$

is uniformly convergent conceming $x$ on $(0, \infty)$, for every fixed $\lambda$ on $\mathfrak{N}_{\lambda_{0}}$.

We assume that we have

$$
\Gamma_{\xi}\left\{\sum_{n=0}^{\infty} \Gamma_{n} g(x+\xi, \lambda)\right\}=\sum_{n=0}^{\infty} \Gamma_{\xi}\left\{\Gamma_{n} g(x+\xi, \lambda)\right\}=g(x, \lambda),
$$

and that, if we put

$$
\Gamma_{n} e^{\lambda x}=g_{n}(\lambda) e^{\lambda x},
$$

we obtain

(2606)

$$
\sum_{n=0}^{\infty} g_{n}(\lambda)=\frac{1}{G(\lambda)}
$$

for every $\lambda$ on $\Re_{\lambda_{0}}$

$3^{\circ}$. We introduce

$$
H_{\lambda_{0}}(x, \lambda)=\sum_{\nu=0}^{k-1} \frac{(-1)^{k-\nu-1} B_{v, \lambda_{0}}^{k}(x) e^{\lambda_{0} x}}{(k-\nu-1) !} \int_{0}^{\infty} t^{k-\nu-1} e^{-\lambda_{0} t} g(t, \lambda) d t
$$

for every $\lambda$ on $\Re_{\lambda_{0}}\left({ }^{57}\right)$.

(57) When if $k=k\left(\lambda_{0}\right)=0$, that is, $G\left(\lambda_{0}\right) \neq 0$, then we put $H_{\lambda_{0}}(x, \lambda) \equiv 0,(0 \leqq x<\infty)$. 
$4^{\circ}$. Finally we introduce

(26.08)

$$
F(x, \lambda)=H_{\lambda_{0}}(x, \lambda)+\sum_{t=0}^{\infty} \Gamma_{n} g(x, \lambda)
$$

Definition. XIII. If

$$
\lim _{\lambda \rightarrow \lambda_{0}} F(x, \lambda), \quad\left(\lambda \varepsilon \Re_{\lambda_{0}}\right)
$$

exists and tends uniformly to a limiting function in any finite interval of $[0, \infty]$, then we call this limiting function the principal solution of the linear translatable functional equation (26.01) with respect to the function-set $\{g(x, \lambda)\}$ and the approximation of $\Gamma^{-1}$ by

$$
\Gamma^{-1}=\sum_{n=0}^{\infty} \Gamma_{n}
$$

in symbolic notation at the point $\lambda=\lambda_{0}$.

Example 1. If specially the operator $\Gamma$ reduces to

$$
\Delta f(x) \equiv f(x+1)-f(x)
$$

and if $\lambda_{0}=0$, then $k=1$, and we may put

$$
\begin{gathered}
\Gamma_{n} g(x, \lambda) \equiv-g(x+n, \lambda), \quad(n=0,1,2, \ldots) \\
H_{\lambda_{0}}(x, \lambda)=\int_{0}^{\infty} g(t, \lambda) d t
\end{gathered}
$$

and consequently

$$
F(x, \lambda)=\int_{0}^{\infty} g(t, \lambda) d t-\sum_{n=0}^{\infty} g(x+n, \lambda)
$$

which shows that our definition is essentially identical with that of Nörlund.

Example 2. If specially the operator $\Gamma$ is given by

$$
\mathrm{E} f(x) \equiv \sum_{i=0}^{m} A_{i} f\left(x+\alpha_{i}\right)
$$

where we assume $0=\alpha_{0}<\alpha_{1}<. \quad<\alpha_{n-1}<\alpha_{n}$ and $A_{0}=1\left({ }^{58}\right)$, then we may take, aftor Ghermanesco,

$$
\Gamma_{n} g(x, \lambda) \equiv(-1)^{n}\left(E_{x}-1\right)^{n} g(x, \lambda),
$$

and consequently

$$
F(x, \lambda)=H_{\lambda_{0}}(x, \lambda)+\sum_{n=0}^{\infty}(-1)^{n}\left(E_{x}-1\right)^{n} g(x, \lambda) .
$$

It may be convenient to give

Definition. XIV. If specially

$$
g(x, \lambda)=g(x) e^{\left(\lambda-\lambda_{0}\right) x},
$$

and $\Re_{\lambda_{0}}$ contains a domain defined by

(58) This makes no essential restriction to generality. 
(26.19)

$$
\begin{cases}1 . & 0<\left|\lambda-\lambda_{0}\right|<\rho, \quad(\rho<0) \\ \text { 2. } & \frac{\pi}{2}<\operatorname{Arg}\left(\lambda-\lambda_{0}\right)<3 \frac{\pi}{2},\end{cases}
$$

then the principal solution defined by this function-set and $\left\{\Gamma_{n}\right\}$ will be denoted by

$$
\mathbb{S}_{0}^{x} g(t) d \Gamma_{t}^{\lambda_{0}}, \quad\left\{\Gamma_{n}\right\}
$$

We call (26.20) the sum of $g(t)$ at the point $\lambda_{0}$.

As the consequences of these definitions we obtain the following fundamental

Theorem XXVI. I. If, for exery $\lambda$ of the set $\mathfrak{R}_{\lambda_{0}}$, the series

$$
\sum_{n=0}^{\infty} \Gamma_{n} \frac{x^{s}}{s !} e^{\lambda_{0} x} \equiv \sum_{n=0}^{\infty} \Gamma_{n, \xi}\left\{\frac{(x+\xi)^{s}}{s !} e^{\lambda_{0}(x+\xi)}\right\}
$$

is uniformly convergent concerning $x$ in any finite range of $(0, \infty)$, then we have

$$
\mathbb{S}_{0}^{x} \frac{t^{s}}{s !} e^{\lambda_{0} t} d \Gamma_{t}^{\lambda_{0}}=B_{k+s, \lambda_{0}}^{k}(x) e^{\lambda_{0} x}, \quad\left\{\Gamma_{n}\right\}
$$

Proof. First let $s=0$. Then, if we put

$$
g(x, \lambda)=e^{\lambda_{0} x} \cdot e^{\left(\lambda-\lambda_{0}\right) x}
$$

then, according to the definitions (26.03)-(26.08), we have

$$
\begin{aligned}
F(x, \lambda)= & \sum_{\nu=0}^{k-1} \frac{(-1)^{k-\nu-1} B_{v, \lambda_{0}}^{k}(x) e^{\lambda_{0} x}-\int_{0}^{\infty} t^{k-\nu-1} e^{\left(\lambda-\lambda_{0}\right) t} d t}{(k-v-1) !} \\
& +\sum_{n=0}^{\infty} \Gamma_{n} g(x, \lambda) \\
= & -\sum_{\nu=0}^{k-1} \frac{B_{n, \lambda_{0}}^{k}(x) e^{\lambda_{0} v}}{\left(\lambda-\lambda_{0}\right)^{k-\nu}}+\frac{e^{\lambda x}}{G(\lambda)}
\end{aligned}
$$

Consequently we have

(26.25) $\lim _{\lambda \rightarrow \lambda_{0}} F(x, \lambda)=\lim _{\lambda \rightarrow \lambda_{0}} \sum_{s=0}^{\infty} B_{k+1, \lambda_{0}}^{k}(x) e^{\lambda_{0} x}\left(\lambda-\lambda_{0}\right)^{s}=B_{k, \lambda_{0}}^{k}(x) e^{\lambda_{0} x}$, that is, (26.19) holds when $s=0$.

Next let $s \geqq 1$. Then, if we define

$$
g(x, \lambda) \equiv \frac{x^{s}}{s !} e^{\lambda_{0} x} \cdot e^{\left(\lambda-\lambda_{0}\right) x}=\frac{x^{s}}{s !} e^{\lambda x},
$$

then, according to the definitions (26.03)-(26.08),

$$
\begin{aligned}
F(x, \lambda)= & \sum_{v=0}^{k-1} \frac{(-1)^{k-\nu-1} B_{v, \lambda_{0}}^{k}(x) e^{\lambda_{0} x}}{(k-\nu-1) !} \int_{0}^{\infty} t^{k-v-\frac{t^{s}}{s !}} e^{\left(\lambda-\lambda_{0}\right) t} d t \\
& +\sum_{n=1}^{\infty} \Gamma_{n} \frac{t^{s}}{s !} e^{\lambda t}
\end{aligned}
$$


which, however, is equal to the equation which may be obtained by formal differentiating (26.24) and then multiplying by $\frac{1}{s !}$.

Here let it bo noticed that the formal differentiations are justified by the assumption that the series (26.21) are uniformly convergent.

Thus we know that if $g(x, \lambda)$ are given by (26.26), the corresponding $F(x, \lambda)$ is given by

$$
F(x, \lambda)=\sum_{\nu=s} \frac{\nu !}{(\nu-s) ! s !} B_{k+\nu, \lambda_{0}}^{k}(x) e^{\lambda_{0} x}\left(\lambda-\lambda_{0}\right)^{\nu-s},
$$

and consequently

$$
\lim _{\lambda \rightarrow \lambda_{0}} F(x, \lambda)=B_{k+r, \lambda_{0}}^{k}(x) e^{\lambda_{0} x},
$$

in any finite rango of $x$ in $[0, \infty)$.

\section{§27. Particular Solution of Non-homogenous Linear Translatable Functional Equation.}

1. In the previous paragraph we have defined the principal solutions and the sums, which may be recognised as at least one of the natural generalisation of those of Nörlund's in calculus of differences.

But, as easily seen, there are many difficulties to express their forms.

For example, the choice of the family of the operators $\left\{\Gamma_{i k}\right\}$ is difficult to be determined.

Therefore wo need the solutions which may be more restrictive than the principal solutions in $\$ 26$, but which have the form to be expressed easily.

For this object there are particular solutions, which are introduced in the papers of Bochner [2], Ghermanesco [1] and Delsarte [1] for the spocial linear translatable functional equations.

In virtue of the summation-formula we are able to extend their ideas to the general equation (26.01) as follows:

Definition XV. Under the assumptions of Theorem XXV.I., let $F(x)$ be defined by

$$
\begin{aligned}
F(x+h)= & \sum_{j=0}^{m+b_{0}} B_{j, \lambda_{0}}^{k}(h)\left(D_{x}-\lambda_{0}\right)^{j} \phi(x) \\
& +\int_{1)}^{\infty} \dot{B}_{m+k, \lambda_{0}}^{k}(h-t)\left\{\left(D_{x}-\lambda_{v}\right)^{m+1} g^{(m+1)}(x+t)\right\} d t .
\end{aligned}
$$

We call this function the particular solution of (26.01) at the point $\lambda_{0}$ with index $m$.

2. Thus defining the principal solutions and the particular solutions, we will give the following:

Theorem. XXVII. I. Let it be assumed that, in Definition XIII, 
(27.02)

$$
\Gamma_{n} \equiv(-1)^{n}(\Gamma-1)^{n} \quad(n=0,1,2, \ldots)
$$

and that $F(x, \lambda)$ and $g(x, \lambda)$ satisfy the assumption of Theorem XXIV.I. for each $\lambda$ of $\Re_{\lambda_{0}}$. Further, putting

$$
g(x, \lambda)=\left(D_{x}-\lambda_{0}\right)^{k} f(x, \lambda)
$$

let us assume that

(27.04)

$$
\lim _{\substack{\lambda \rightarrow \lambda_{0} \\ \lambda \text { on } \Re_{\lambda_{0}}}}\left(D_{x}-\lambda_{0}\right)^{j} f(x, \lambda)=\left(D_{x}-\lambda_{\cup}\right)^{j} f(x)
$$

where we have put

$$
g(x)=\left(D_{x}-\lambda_{0}\right)^{k} f(x)
$$

Then the necessary and sufficient condition that the principal solution defined in Definition XIII coincides with the particular solution defined in this paragraph is to satify, for any $\lambda$ of $\Re_{\lambda_{0}}$,

(27.06) $\lim _{n \rightarrow \infty}(-1)^{n}(\underset{x}{\Gamma}-1)^{n}\left(D_{x}-\lambda_{0}\right)^{j} f(x, \lambda)=0,(j=0,1, \ldots, k+m+1)$.

and that

$(27.07)$

$$
\begin{gathered}
\lim _{\substack{\lambda \rightarrow i_{0} \\
\lambda \text { on } \eta_{\lambda_{0}}}} \int_{0}^{\infty} \dot{B}_{m+k, \lambda_{0}}^{k}(h-t)\left(D_{x}-\lambda_{0}\right)^{m+1} g(x+t, \lambda) d t \\
=\int_{0}^{\infty} \dot{B}_{m+k, \lambda_{0}}^{k}(h-t)\left(D_{x}-\lambda_{0}\right)^{m+1} g(x+t) d t .
\end{gathered}
$$

Proof. By the assumption we have

$$
\left.F(x, \lambda)=H_{\lambda_{0}}(x, \lambda)+\sum_{n=1}^{\infty}(-1)^{n} \underset{x}{\Gamma}-1\right)^{n} g(x, \lambda),
$$

and therefore

$$
\text { (27.09) } \begin{aligned}
\Gamma_{0}(F(x, \lambda)) & \left.\left.=\underset{0}{\Gamma}\left(H_{\lambda_{\theta}}(x, \lambda)\right)+\underset{0}{\Gamma}-1+1\right) \sum_{n=0}^{\infty}(-1)^{n} \underset{0}{\Gamma}-1\right)^{n} g(x, \lambda) \\
& =g(x, \lambda)+\lim _{n \rightarrow \infty}(-1)^{n} \underset{x}{\Gamma-1)^{n} g(x, \lambda) .}
\end{aligned}
$$

Thus we obtain view of the summation formula (25.04)

(27.10) $\quad F(x+h, \lambda)$

$$
\begin{aligned}
= & \sum_{j=0}^{m+k} B_{j, \lambda_{0}}^{k}(h) e^{\lambda_{0} h}\left(D_{x}-\lambda_{0}\right)^{j} f(x, \lambda) \\
& +\sum_{j=1}^{n+k} B_{j, \lambda_{0}}^{k}(h) e^{\lambda_{0} h} \lim _{n \rightarrow \infty}\left\{(-1)^{n}(\underset{x}{\Gamma}-1)^{n+1}\left(D_{x}-\lambda_{0}\right)^{n} f(x, \lambda)\right\} \\
& +\int_{0}^{\infty} \dot{B}_{m+k, \lambda_{0}}^{k}(h-t)\left(D_{x}-\lambda_{0}\right)^{m+1} g(x+t, \lambda) d t \\
& +\int_{0}^{\infty} \dot{B}_{m+k, \lambda_{0}}^{k}(h-t) \lim _{n \rightarrow \infty}\left\{(-1)^{n}(\Gamma-1)^{n+1}\left(D_{x}-\lambda_{0}\right)^{m+1} g(x+t, \lambda)\right\} d t .
\end{aligned}
$$

This last relation leads us to the consequence to be proved. It will be 
remarked that the theorem may be recognised as a generalisation of the theorem of Ghermanesco $\left({ }^{59}\right)$, and that, for some special linear translatable operators, it may be able to deduce more decided condition for $f(x, \lambda)$ from (27.06), as Ghermanesco [1] has done in the case of the functional equation (0.34).

We will not enter into the discussion of the principal solution and the particular solution in details, but we wish merely remark that the Bochner's composition theorem of particular solutions will be also easily verified after some easy modification. See Appendix III.

\section{Bibliography。}

This biliography attempts to bring together the papers eited in the text and the literatures closely related to our subject such as those about operational ealcalss and theory of functionals.

1. Adams, C. R., [1] Linear $q$-difference equations. Bull. Am. Math. Soc., 37 (1931). (This includes a list of memoirs about difference-equation and related subject published during the time from 1924 until 1931).

2. Banach, S. [1] Théorie des opérations linéaires, Warszawa (1932).

3. Benz, E. [1] Über lineaire, verschiebungstraue Funktional-operationen und die Nullstellen ganzer Funktionen. Comment. Math. Helvetici, 7 (1935).

4. Besicovitch, A. [1] Almost periodic functions. Cambridge, (1932!.

5. Birkhoff, G. D. and W. T. Trjitzinsky [1] Analytic theorey of singular differene equations. Acta Math., 60 (1933).

6. Blumenthal, L. M. [1] Note on Fractional operators and the theory of composition. Am. Journ. Math., 53 (1931).

7. Bochner, S. [1] Über eine Klasse singulärer Integralgleichungen. Sitzber. d. Preuss. Acad. d. W. Phys-Math., Klasse, XII (1930.

[2] Hauptlösungen von Differenzengleichungen. Acta Math., 51 (1928).

[3] Allgemeine lineare Differenzengleichungen mit asymptotisch konstanten Koeffizienten. Math. Zeistschr, 93 (1981).

[4] Über gewisse Differential.und allgemeinere Gleichungen, deren Lösungen fast periodish sind. I. Teil. Der Existenzsatz. Math. Ann., 102 (1929). II. Teil. Der Beschränktheitssatz. Math. Ann., 103 (1930). III. Teil. Systeme von Gleichungen. Math. Ann., $104(1931)$.

[5] Vorlesungen über Fouriersche Integrale. Leipzig, (1932).

[6] Ein Satz ïber lineare operationen. Math., Zeitschr., 29 (1929).

[7] Spektralzerlegung linerer Scharen unitären Opeatoren. Sitzber. d. Preuss. Acad. d. W. Phys-Math. Klasse (1932).

8. Bohr, H. [1] Zur Theorie der fastpersodischen Funktionen. I, II, ]II. Acta Math., 45, (1924); 46 (1925); 47 (1926).

9. Bourlet, C. [1] Sur les opérations en général et les équations differentiélles linéaires d'ordre infini. Ann. L'Ecole Norm. 3rd ser. 14 (1897).

[?] Sur certaines équations analogues aux différentielles. Ann. L'Eco'e Norm. 3rd. ser., 16 (1899).

( 59$)$ Ghermanesco, [5], §13, p. $262-264$.

$\left.{ }^{60}\right)$ Bochner, [2], $\$ 4$, p. $16-24$. 
[3] Sur les transmutations. Bulltein de Soc. Math., 25 (1897).

10. Bromwich, T. J. I. A. [1] Normal coordinates in dynamical systems. Proc. London Math. Soc., 15 (2) (1916).

[2] A New Method for solving two dimensional problems of physical types. Proc. London Math. Soc., 30 (1929).

[3] An application of Heaviside's Methods to viscous fluid motion. Journ. London Math. soc., 5 (1930).

11. Bush, V. [1] Note on operational calculus. Journ. Math. Phys, M. I. T., 3 (1924).

[2] Operational circuit analysis. New York (1929).

12. Carmichael, R. D. [1] Systems of linear difference equations and expansions in series of exponential functions. Trans. Am. Math. Soc., 35 (1933).

[9] Summation of functions of a complex variable. Annals. Math. 34 (1933).

[3] Functions of exponential type. Bul. Am. Math. Soc, 40 (1934).

13. Carson, J. R. [1] The Heaviside operational Calculus. Bull. Am. Math., Soc., 32 (1926).

[2] Eiectric eircuit the and the operational Calculus. New York. (1926).

14. Cauchy, A. L. [1] Mémoire sur l'intégration des êquations linéaires aux différences partialles et à coefficients consts. Journ. Ec. Polyt. cah., 19 (1823).

[2] Mémoire sur l'application du calcul des résidus á la solution des problèmes de physique mathématique. Paris (1837).

15. Davis, H. T. [1] Euler differential equation of infinite order. Am. Math. Soc. Monthly. 32 (1925).

[2] The application of fractional operators to functional equations. Am. Journ. Math., 49 (1927).

[3] Differential equations of infinite order with constant coefficients. Am. Journ. Math., $52(1930)$

[4] The Laplace differential equation of infinite order. Annals, Math. 32 (1931).

16. Delisarte, J. [1] Les fonctions moyenne-périodiques. C. R. Acad. Sci, Paris, 198 (1934).

[2] Application de la théories des fonctions moyenne-périodiques à la résolution de certaines équations intégrales. C. R.'Acad. Sci. Paris, 198 (1934).

[3] Sur un princpie général de developpement des fonctions d'une variable réelle en séries de fonctions entieres. C. R. Acad. Sci. Paries, 200 (1985).

[4] Sur l'application d'un principe général de déveoloppement des fonctions d'une vaiable, aux séries de fonctions de Bessel. C. R. Acad. Sci., Paris 200, (1935).

[5] Les fonctions "moyenne-périodiques." Journ. d. mathém. pures et appliquées, neuvième sêrie, tome quatorziéeme, (1935).

1\%. Dixon, A. C. [1] The theory of a thin elastic plate, bounded by two circular arcs and clamped. Proc. London Math. Soc., Ser., 2, 19 (1921).

[2] Expansions and functions reduced to zero by the operator sinhD-cD. Proc. London Math, Soc. Ser. 2. 21. (1923).

18. Doetsch, G. [1] Die Integrodifferentialgleichungen vom Faltungstypus. Math. Ann., 89 (1923).

[2] Über das Problem der Wärmeleitung. Jahresber. d. D. M. V., 33 (1933)

[3] Die Anwendung von Funktionaltransformationen in der Theorie der Differentialgleichungen und die symbolische Methode (Operatorenkalkül). Jahresber. d. D. M. V., 43 (1934). (This contains a biliograph of the subject).

19. Fantappiè, L. [1] Überblick über die Theorie der analytischen Funktionale und ihre Anwendungen. Jahresber. d. D. M. V., 43 (1934). (This contains a biliography of the 
numerous works of the author on this subject).

20. Fejes, L. [1] Des séries exponentielles de Cauchy. C. R. Paris, 200, (1935).

21. Filton, L. N. G. [1] On the expansion of polynomial sin series of functions. Proc. London Math. Soc, 2 ser. 4 (1907).

22. Flament, F. [1] La notion de continuité dans l'étude des transmutations distributives des fonctions d'une variable complexe et ses applications. I, II, III, Bulletin sc. Math., (2) 52 (1998).

[2] Le développement d'une transmutation linéaire en série de puissances de la derivation et l'extension d'une transmutation distributive C. R. Paris, 184 (1927).

[3] La notion de transmutation dérivée dans l'étude des transmutations linéaires. Atti del Congresso dei Math, (Bologra) 3. (1928).

[4] Le développement d'une transmutation linéaire par rapport á la differentiation finie. Rendic. d. Parlemo, 54 (1930).

23. Fry, T. C. [1] The solution of circuit problems Phys. Reviw. 14, II. ser. (1919).

[2] The application of modern theory of integration to the solution of differential equations. Annals Math., 22 (1920-1921).

24. Geppert, H. [1] Über eine Verallgemeinerung der linearen Randwert-probleme. Math. Ann., 95 (1926).

[2] Entwicklungen willkürlicher Funktionen nach funktiontheoretischen Metboden. Math. Ztschr., 20 (1924).

25. Ghermanesco, M. [1] Sur les équations aux différences finies. Atti Acad. naz. Lincei, Rend., VI. s. 17 (1933).

[2] Sur l'equationelle linéaire d'ordre $p$ à coefficiente constants. Bull. Acad. Roy. Belg., V. s. 19 (1933).

[3] Sur les équations aux différences finies. C. R. Acad. Sci. Paris, 197 (1933).

[4] Sur un système d'équations á une infinité d'inconnus. C. R. Acad. Paris, 198 (1934).

[5] Sur les équations aux différence finies. Acta Math., 62 (1934).

[6] Sur les équations aux differences finiés. Annali di Math. Pure e. Appl., Ser. IV, 12 (1934).

26. Hardy, G. H. and Titchmarsh, E. C. [1] Solutions of some integral equations considered by Bateman, Kapteyn, Littlewood and Milne. Proc. London Math. Soc., (2), 23 (1923).

[2] Correction of an error in a paper on certain integral equations. Proc. London Math. Soc., (2) 24 (1935) XXX i-XXX iii (Records for 12 February 1925).

[3] New Solution of an integral equation. Proc. London Math. Soc. (2) 41 (1936).

27. Heaviside, O. [1] On Operators in Physical Mathematics. Roy. Soc., 52 (1893) 54 (1893).

28. Hellinger, E. und Toeplitz, O. [1] Integralgleichungen und Gleichungen mit unendlichvielen Unbekannten. Enc. Math. Wiss. II C 13. (1927) § 24. Linear Funktionaloperationen (Specially p. 1478-1481).

29. Hilb, E. [1] Zur Theorie der linearen funktionalen Differentialgleichung. Math. Ann., 78 (1918).

[2] Lineare Differentialglichungen unendlich hoher Ordnung mit ganzen rationalen Koeffizienten. Math. Ann., 82 (1921); 84 (1921).

[3] Zur Theorie der linearen Differenzengleichungen. Math. Ann., 85 (1922).

[4] Zur Theorie der linearen Differenzengleichungen. I, Math. Ztschr., 14 (1922), II, id, 15 (1922), III, id. 19 (1924).

30 Hoheisel, G. [1] Lineare funktionale Differentialgleichungen. I. Mitteil. und II. Mitteil. Math. Ztzchr., 14 (1922). 
31. Hopf, E. [1] Über lineare Integralgleichungen mit positive Kern. Sitzuugsber, Akad. Berlin (1928).

32. Hopf, E. and M, Wiener. [1] Über eine Klasse singulärer Integralgleichungen Sitzungsber. d. Preuss. Akad., Math.-Physikalische Klasse, (1931).

33. Izumi, S. [1] On some integral equatians. Proc. Imp. Acad. Jap., XI (1935).

34. Izumi, S. and T, Kitagawa. [1] On some integral equations. Jap. Journ. Math., XII (1935).

[2] On some linear operations, Tôhoku Math. Journ., 40 (1936).

[3] On some integrai equations. II. Tôhoku Sc. Reports. Sendai (1936).

35. Jeffreys, H. [1] Operational Methods in mathematical physics. Camb. Univ. Press. Second Edition (1931).

36. Kitagawa, T. [1] Nagumo si no Mondai ni tuite, (I). Zenkoku Sizyo Danwakai, 45 (July, 18th) (1935); (II) ibid. 46 (July, 25th) (1935).

[2] On the expansion of arbitrary function with respect to linear translable functional transformation. Proc. Physico-Nath. Soc. Jap., (III) 17 (1935).

[3] Arusyu no Senzyo Idôkanô Kansûhôteisiki ni tuite, (I). Zenkoku Sizyo Danwakai, 59 (September, 27th) (1935); (II) ibid. 60 (October, 4th) (1935); (III) ibid. 61 (October, 11th) (1935).

[4] Senzyo Kansen Sayôsi ni kansuru Tenkaimondai. Zenkoku Sizyo Danwakaj, 65 (November, 18th) (1935); (II) ibid. 66 (December, 15th) (1935).

37. Koch, H. v. [1] Sur les équations differentielle linéaires d'ordre infini. Ark. f. mat, 16 (1922).

38. Kostitzin, V. A. [1] Sur les solutions singulières des équations iniegrales du cycle fermé. Rec. Math. Moscou, 35 (1926).

[2] Sur les solutions singulières des équations intégrales de Volterra. C. R. Acad. Sci. Paris, 184 (19:7).

[3] Sur une équation intégro-différentiélle. C. R. Acad. Sci, Paris, 186 (1928).

39. Langer, R. E. [1] On the zeros of exponential snms and integrals. Bull. Am. Math. Soc., 37 (1931).

40. Lettenmeyer, [1] Über das asymptotisohe Verhalten der Losungen von Differenzengleichungen und Systemen von Solchen. Sitzber., München, (1980).

41. Lévy, P. [1] Leçons d'analyse fonctionnelie. Paris (1922).

[2] Sur la dérivation et l'intégration généralisées. Bull. d sci. Math., II ser. 49 (1923).

[3] Le calcul symbolique d'Heaviside. Bull. d. scienc. math., 50 (II ser). (1926).

42. Mächler, W. [1] Laplace Integraltransformation and Integration partieller Differentialg'eichungen vom hyperbolischen and parabolischen Typus (Ein Beitrage zum Heavisideschen Operatoren-kalkül) Comment. math. helv., 5 (1933).

43. Martis, S. [1] Studio della Transformazione di Laplace e della sua inversa del punto di vista dei funzionali analytici. Rendic. d. C. M. d. Parlemo, Tome LVII (1933).

44. Milne-Thompson, L. M. [1] The calculus of Finite Differences. (1930).

45. Nagumo, M. [1] Kansûhôteisiki $\int f(x+t) d \varphi(t)=0$. Zenkoku Sizyodanwakai, 34 (March, 20th) (1935).

46. Nagumo, M. and S. Kakutani. [1] (with Y. Mino) Kansûhôteisiki $f(x)$ $=\frac{1}{2} \int_{x-1}^{x+1} f(t) d t$ ni tuite. Zenkoku Sizyodanwnkai, 21(November, 27th) (1934); [II] ibid. 22 (December, 4tS) (1934).

47. Neuman, J. v. [1] Allgemeine Eigenwerttheorie Hermitescher Funktionalopera- 
toren. Math. Math. Ainn., 102 (1929).

[2] Über E'unktionen von Funktionaloperatoren, Ann. of Math., 32 (1931).

48. Nörlund, N. E. [1] Mémoire sur le calcul aux differences. Acta Math., 44 (1922).

[2] Differenzenrechnung, Spring (1924). (This book contains a bibliograph of the papers on the subject until 1924).

49. Oltranmare, G. [1] Calcul de généralisation. Paris (1899).

50. Paley, R.E. A. C. and N. Wiener [1] Fourier transforms in the complex domain. Am. Math. Sce. Colloq. Publ, Vol. XIX (1934).

51. Pennel, W. O. [1] A General Operational Analysis. Journ. of Math. Massachusetts, 7 (1926).

52. Perron, O. [1] Über Stablität und asymptotisches Verhalten der Lösungen eines Systems endlicher Differenzengleichungen. Journ. f. Math., 161 (1929).

[3] Über Stabilität und asymptotisches Verhalten der Integrale von gewöhnlichen Differenzengleichungen. Atti d. Congresso Int. d. Math. (Bologna), 3 (1930).

[3] Über Differentialgleichungen unendlich hoher Ordnung mit ganzen rationa'en Koeffizienten, I, II, III. Math. Ann., 82; 84 (1920, 1921).

[4] Über Summengieichungen und Poincarèsche Differenzengleichungen, Math. Ann., 84 (1821).

[5] Lineare Differentialgleichungen unendlich hoher Ordnung mit ganzen rationaler Koeffizienten. Math. Ann., 84 (1921).

53. Pincherle, S. [1] (With Amaldi) Le operazioni distributive e le loro applicazioni a!l analyisi. Bologna (1901).

[2] Funktional operationen und-gleichungen. Enc. Math. Wiss., II. 1. Teil, A 11 (1905) (also E'quations et opérations fonctionnelles. Enc. des sciences math., II vol. 5, fasc. 1.)

[3] Sur la résolution de l'equation fonctionnalle $\sum h_{v} \varphi\left(x+a_{v}\right)$ á coefficients constant. Acta Math., 48 (1926).

[4] Sulle operationi distributive commutabili c,n una operazione data. Atti d. R. Accad. Torino, 30 (1895).

[5] Una classe speciale di forme differenziali lineari d'ordine infinite. Memorie dell'. Acead. di Bologna 4 (8th series) (1927).

[6] Sullo scarto dalla permutabilitá nelle operatzioni lineari. Rendic. dell' Aead. di Bologna 35 (1931).

[7] Un' applicazione del metodo simlolico. Bull. della Unione Math., 11 (1932).

54. Pòlya, G. [1] Eine Verallgemeinerung des Fabryschen Satzes. Göttingen Nachrichten (1927).

[2] Sur certaines transformations functionelles linéaires des fonctions analytiques. Bull. Soc. Math. France, 52 (1924).

55. Raclis, R. N. [1] Solution principle de l'équation linéaire anx differences finis, A.cta Math., 55 (1939).

56. Reid, M. [1] Properties of solutions of an infinite system of ordinary linear differential equations of the first order with auxiliary boundary conditions. Trans Am. Math. Soc., 32 (1930).

57. Rellich, F. [1] Spektraltheorie in nichtseparabeln Räumen. Math. Ann., 110 (1925).

58. Riesz, F. [1] Sur les fonctions des transformations hermitiennes dans l'espace de Hilbert. Acta Szeged, VII. (1935).

[2] Sur les opérations fonctionnelies linéaires. C. R. Accad. Sci. 149 (1909).

[3] Über lineare Funk ionalgleichung. Acta Math. 41 (1918). 
59. Riesz, M. [1] Les fonctions conjugnés et les séries de Fourier. C. R. Acead. Sci. Pa.is. 178 (1924)

[2] Sur les fonctions conjuguées. Math. Ztschr., 27 (198).

60. Ritt, J. F. [1] On a general class of linear homogeneous differential equations of infinite order with constant coefficients. Trans. Am. Math. Soc., 18 (1917).

61. Sbrana, F. [1] Sopra un gruppo di operatori fnnzionali che interessanoda Fisica. Annali d. Math, (4th ser). 7 (1929).

[2] Sull' operazione infinitesimale nel gruppo delle derivazioni. Atti dei Lincei 11, (1930).

[3] Sopra un notevole gruppo di operatiori funzionali. Rend. Afcad. Lincei, 8 (6th series) (1928).

[4] Propriétá caratteristiche dell' operazione infinitesimale nel gruppo delle derivazioni. Rend, dell' Acead. dei lincei, 11 (6th séries) (1930).

62. Schürer, F. [1] Eine gemeinsame Methode zur Behandlung gewisser Funktionalgleichungsprobleme. Leipziger Ber., (1918), (This contains a list of his papers about the subjects).

63. Sheffer, I. M. [1] Linear differential equations of infinite order with polynomial coefficients of degree one. Annals Math., 30 (1929).

[2] Systems of linear differential equation of infinite order with constant coefficients. Annals Math. 30 (1929).

[3] Expansions in generalised Apell polynomials and a class of related liner functional equations. Trans. Amer. Math. Soc., 31 (1929).

[4] Systems of infinitely many linear differential equations of infinite order with constant coefficients. Trans. Amer. Math Soc., 31 (1929).

64. Smith, J. J, [1] Heaviside's operators and contour integrals. Atti Congr. Intern. Math., Bologna, 5 (1928).

65. Späth, H. [1] Uber das asymptotische Verhalten der Lösungen nichthomogener linearer Differenzenglelchungen. Acta Math., 51, (1927).

66. Stacho, T. v. [1] Operations kalkül von Heaviside und Laplace Transtormation. Acta Szeged, 3 (1926!.

67. Stone, M. H. [1] Linear Transformations in Hilbert space and their applications to analysis. Chapter VI. Am. Math. Soc. Colloq. Publ., XV. (1932).

68. Straneo. St. L. [1] Formula di transformatione per le valutazioni degli operatori funzionali. Bollettino V. M. I. 7. (1928).

[2] Metoda funzionale applicato alla risoluzione di problemi sulla progagazione del calore. Atti Pontifica Accad., 81. (1928).

69. Szegî, G. [1] Ein Beispiel zo Nörnnds Summationsverfabren. Annals Math., 34 (1933).

70. Tamarkin, J. [1] Some general problems of the theorey of ordinary linear differential equations and expansion of an arbitrary function in series of fundamental functions. Math. Ztschr., 27 (1928).

[2] On Volterra's integro-functional equation. Trans. Am. Math. Soc., 28 (1926).

71. Titchmarsh, E. C. [1] The zeros of certain integral fnnetions. Pree, London Math. Soc., 25 (1926).

72. Valiron, G. [1] Sur les solutions des equations differentielles lineaires d'ordre infini et a coefficients constants. Ann. L'Ecole Norm., 46 (1929).

73. Volterra, V. [1] Leçons sur les fonctions de lignes, Paris, Gauther-Villars (1913). 
[2] Theory of functionals and of integral and integro-differential equations, London and Glasgow, Blackie and Son, (1930).

74. Walther, A. [1] Über lineare Differenzengleichungen mit konstanten Koeffizienten und fastperiodischer rechter Seite. Göttingen Nachricht., 27 (1928).

75. Wiener, N. [1] The solution of a difference equation by trigonometrical integrals. Jour. Math. Phys. M. I. T., 4 (1926).

[2] The operational calculus. Math. Ann., 95 (1926).

[3] Generalised Harmonic Analysis. Acta Math., 55 (1930).

[4] Tanberian Theorems. Annals Math., 33 (1932).

76. Wintner, A. [1] Zur Theorie der unendlichen Differential systeme. Math. A.nn., 95 (1926).

[2] Zur Lösung von Differential systemen mit uneudlich vielen Veränderlichen. Wath. Ann. 98 (1927).

[3] Zur Analysis in Hilbertschen Raume. Math. Zeitschr., 28 (1928).

7\%. Young, R. C. [1] The asymptotic behaviour of $\int_{a-0}^{b++\theta} e^{\lambda t} d \varphi(t)$. Math. Ztschr., 40 (1935).

78. Young, W. H. and G. C. Young. [1] On the theorem of Riesz-Fischer. Quart. J. of Math., 44 (1913).

\section{Appendix I}

The connections between our theory of translatable operators and that of linear differential equation of infinite order will be more intimately explained by fixing the domain of the operator as being consisted of functions which are infinitely many time differentiable. In the near future we will give this remark.

\section{Appendix II}

Some of the results obtained in this paper will be generalised to the operators which are permutable with certain fundamental operator possessing certain spectral properties. In the near future we will communicate them in the notes published in the Proceeding of the Imperial Academy of Japan.

\section{Appendix III}

In order to stress the fact that, to each point $\lambda_{0}$ of the complex plane, there correspond the Bernoulli's polynomials, we have not, in Chapter $V$, assumed that $\lambda_{0}$ is zero. This is, however, merely for the cmphasis, and indeed simple transformation of the functional equation will make us sufficient to consider the case $\lambda_{0}=0$ without any loss of gencrality. With respect to the principal solutions, we wish to point out here that we may be permitted to call the solution to be principal when wo would have solved the questions proposed in Introduction. It is the author's conjecture that what wo have called the principal solutions would possess some adequate 
function-theoretical properties which distinguish from any others. It is also without interest to find the condition under which the principal solutions become identical with the so-called sum.

\section{Appendix IV ${ }^{(1)}$}

Recently there published two remarkable literatures:

Carmichael, R. D. [4] Linear Differential Equations of Infinite oder. Bull. Am. Math. Soc., 42 (1936).

Davis, H. T. [5] The Theory of Linear Operators from the Standpoint of Differential Equations of Infinite Order. The Principia Press (1936).

The latter gives us an exhaustive account on the subject. In this book the reader will find a complete bibliography on operational calculus. The object of the former is to give a general outlook on the subject. At its conclusion Carmichael indicated the following fact: "In these resuits of Ritt and Pòlya and Valiron we have properties of the solutions of linear differential equations of infinite order which are different from anything that arises in the finite case.... And yet a single small group of them stands out as apparently unique in the whole literature."

As to this problem we will remark here that the notion of the association of the generating function introduced in our present memoir will play some important part and that the generating function of any differential operator of finite order with coefficients is a polynomial and is never associated to any interval of positive length.

\section{Appendix V}

Recently certain decided results on non-harmonic Fourier's series are given by the following paper.

Levinson, N. On Non-Harmonic Fourier Series. Annals Math., 37 (1936),

Here the author will remark that it will be interesting to connect our results on Cauchy's series with those of Levinson's.

(1) Appendix IV-V are added during the course of the press. (on March 15 th, 1987) 